MAGNITUDE AND FREQUENCY OF FLOODS IN ARKANSAS

By Braxtel L. Neely, Jr.

U.S. GEOLOGICAL SURVEY

Water Resources Investigations Report 86-4335

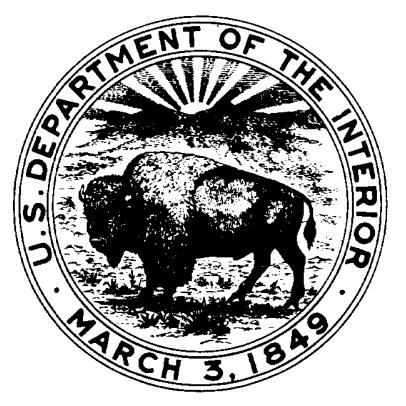

Prepared in cooperation with the

ARKANSAS STATE HIGHWAY AND TRANSPORTATION DEPARTMENT

Little Rock, Arkansas

1987 
UNITED STATES DEPARTMENT OF THE INTERIOR

DONALD PAUL HODEL, Secretary

GEOLOGICAL SURVEY

Dallas L. Peck, Director

For additional information write to:

District Chief

U.S. Geological Survey Water Resources Division 2301 Federal office Building

Little Rock, Arkansas 72201
For purchase write to:

U.S. Geological Survey Books and Open-File Reports Section Federal Center

Box 25425

Denver, Colorado 80225 
Abstract .............................................. $\ldots \ldots 1$

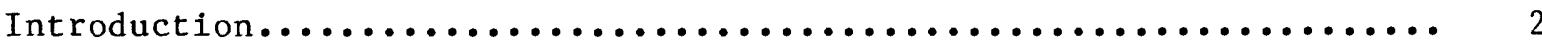

General setting......................................... 4

Flood data.............................................

Flood magnitude and frequency at gaging stations.................

Flood magnitude and frequency at ungaged sites on streams draining

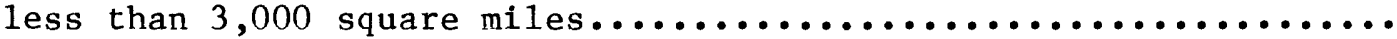

Basin and climatic characteristics......................

Regression analysis................................

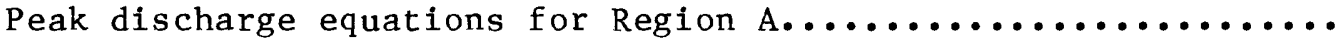

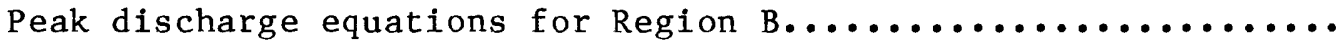

Accuracy of estimating equations......................

Limitations.....................................

Flood magnitude and frequency at or near gaged sites on streams

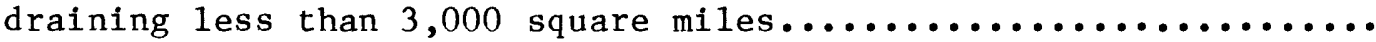

Flood magnitude and frequency at ungaged sites on streams draining

less than 3,000 square miles using the hydraulic radius..........

Estimating the cross section.........................

Stage-discharge relation............................

Discharge-frequency curve...........................

Flood magnitude and frequency on large rivers..................

Mississippi River................................

St. Francis River..................................

Black River.........................................

Arkansas River....................................

White River.......................................

Red River.........................................

Ouachita River.....................................

Flood frequency for urban areas..........................

Conclusions..........................................

Selected References...................................

\section{ILLUSTRATIONS}

Figure 1-5. Maps showing:

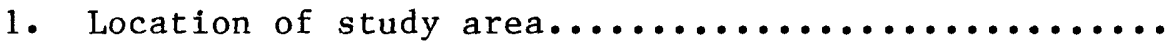

2. Physiographic provinces in Arkansas...............

3. Mean annual precipitation for Arkansas for the base

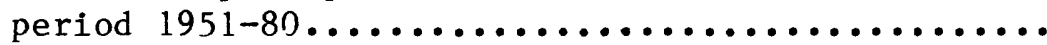

4. Regional boundaries and locations of gaging

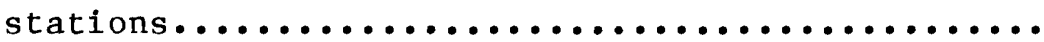

5. Cross section of valley from topographic map.......

6. Graph showing stage-discharge, hydraulic radius-

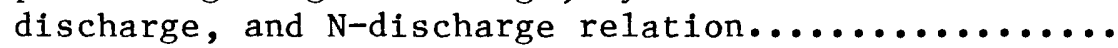

7-9. Graphs showing flood magnitude and frequency curves for:

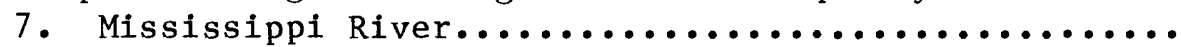

8. St. Francis River........................

9. Black River............................. 
10-13. Graphs showing flood magnitude and frequency curves for:

10. Arkansas River............................ 42

11. White River.............................. 43

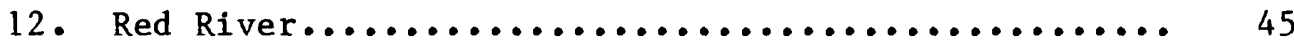

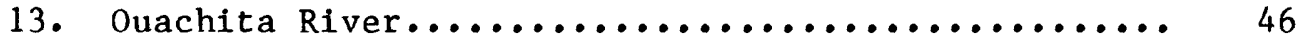

14. Map showing rainfall intensity for the 2-hour, 2-year occurrence...............................

\section{TABLES}

Table 1. Discharge, for selected recurrence intervals, at gaging

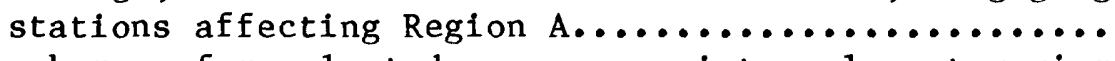

2. Discharge, for selected recurrence intervals, at gaging

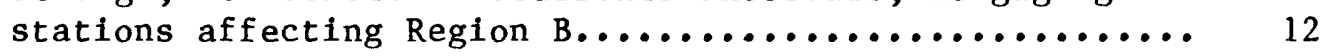

3. Discharge, for selected recurrence intervals, on large

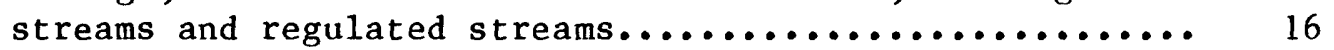

4. Hydraulic radius and $\mathrm{N}$, for selected recurrence interval,

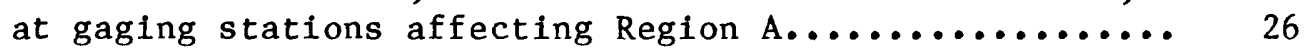

5. Hydraulic radius and $\mathrm{N}$, for selected recurrence interval, at gaging stations affecting Region B............... 27

6. Computation of discharge and cross-sectional properties.... 35 


\section{CONVERSION FACTORS}

For use of readers who prefer to use International System (SI) units, rather than the inch-pound terms used in this report, the following conversion factors may be used.

Mu1tip1y

foot $(\mathrm{ft})$

cubic foot per second $(\mathrm{ft} / \mathrm{s})$

million gallon per day (Mga1/d)

inch (in.)

mile (mi)

square mile $\left(\mathrm{mi}^{2}\right)$

acre/foot (acre-ft)
By

0.3048

0.02832

0.0439

25.4

1.609

2.590

1,233
To obtain

meter (m)

cubic meter per second $\left(\mathrm{m}^{3} / \mathrm{s}\right)$

cubic meter per second $\left(\mathrm{m}^{3} / \mathrm{s}\right)$

millimeter (mm)

kilometer $(\mathrm{km})$

square kilometer $\left(\mathrm{km}^{2}\right)$

cubic meter $\left(\mathrm{m}^{3}\right)$

Sea leve1: In this report "sea leve1" refers to the National Geodetic Vertical Datum of 1929 (NGVD of 1929)--a geodetic datum derived from a general adjustment of the first-order level nets of both the United State and Canada, formerly called "Mean Sea Leve1 of 1929." 


\title{
MAGNITUDE AND FREQUENCY OF FLOODS IN ARKANSAS
}

By Braxtel L. Neely, Jr.

\author{
ABSTRACT
}

Techniques are presented for estimating the magnitude and frequency of peak discharges on streams in Arkansas. Comprehensive regression analyses were made in which physical characteristics of streams were related to flood characteristics at gaging stations. Equations derived from the regression analyses provide estimates of peak discharges with selected recurrence intervals from 2 to 100 years on streams that have drainage areas less than 3,000 square miles. The regression analyses indicate that size of drainage area, slope of the main channel, length of the main channel, elevation of the basin, and mean annual precipitation were the most significant basin and climatic characteristics that affect the magnitude and frequency of floods.

A technique is presented for estimating a stage-discharge relation at ungaged sites based on a cross-section determined from topographic maps. This cross-section and stage-discharge relation are used to determine the hydraulic radius for the discharges for each recurrence interval. Techniques are presented for estimating the magnitude and frequency of peak discharges using the hydraulic radius with other physical characteristics of the basin. The techniques that use the hydraulic radius give standard errors of estimate from 5 to 16 percent lower than techniques that do not use hydraulic radius.

Data from 200 gaging stations with drainage areas less than 3,000 square miles and with at least 10 years of record were used in the analyses.

Large rivers such as the Red, Arkansas, White, Black, St. Francis, Mississippi, and Ouachita Rivers have floodflow characteristics that differ from those of smaller tributary streams and were treated individually. Regional regression equations are not applicable to these large rivers. The magnitude and frequency of floods along these rivers are based on station data.

A method is described for estimating the magnitude and frequency of peak discharges on streams for urban areas in Arkansas. The method is from a nationwide U.S. Geological Survey flood-frequency report which uses urban characteristics to adjust rural discharges to estimate urban discharges.

Annual peak discharges at all gaging stations, which are the basic data that support this analysis, are not published in the report. These data are published in a companion report entitled, "Annual peak discharges and stages through 1984 for gaging stations in Arkansas". 


\section{INTRODUCTION}

The magnitude and frequency of floods are primary factors in the design of bridges, culverts, streets, embankments, dams, levees, and other structures near streams. Information on flood magnitude and frequency is used in managing flood plains, planning subdivisions, and in establishing flood insurance rates.

The Arkansas State Highway and Transportation Department is fully aware of the need for adequate flood peak data to more efficiently design drainage structures in Arkansas. Because of this need, the Arkansas State Highway and Transporation Department entered into a cooperative agreement with the U.S. Geological Survey to update a previous flood-frequency report based on data collected through 1984. This flood-frequency report supersedes the first two reports for Arkansas prepared by Patterson $(1961,1971)$ because of additional available data and new analysis techniques.

The purpose of this report is to provide a method of estimating the magnitude of floods with selected recurrence intervals from 2 to 100 years for streams in Arkansas. Equations for streams that have drainage areas less than 3,000 square miles $\left(\mathrm{mi}^{2}\right)$ were developed by the multiple regression technique. Equations for urban areas are from the report by Sauer and others (1983). For larger streams, individual station analysis is provided.

The study area is Arkansas, however data collected outside the State on streams that drain into and from Arkansas are considered. Figure 1 is a general location map of the study area. 


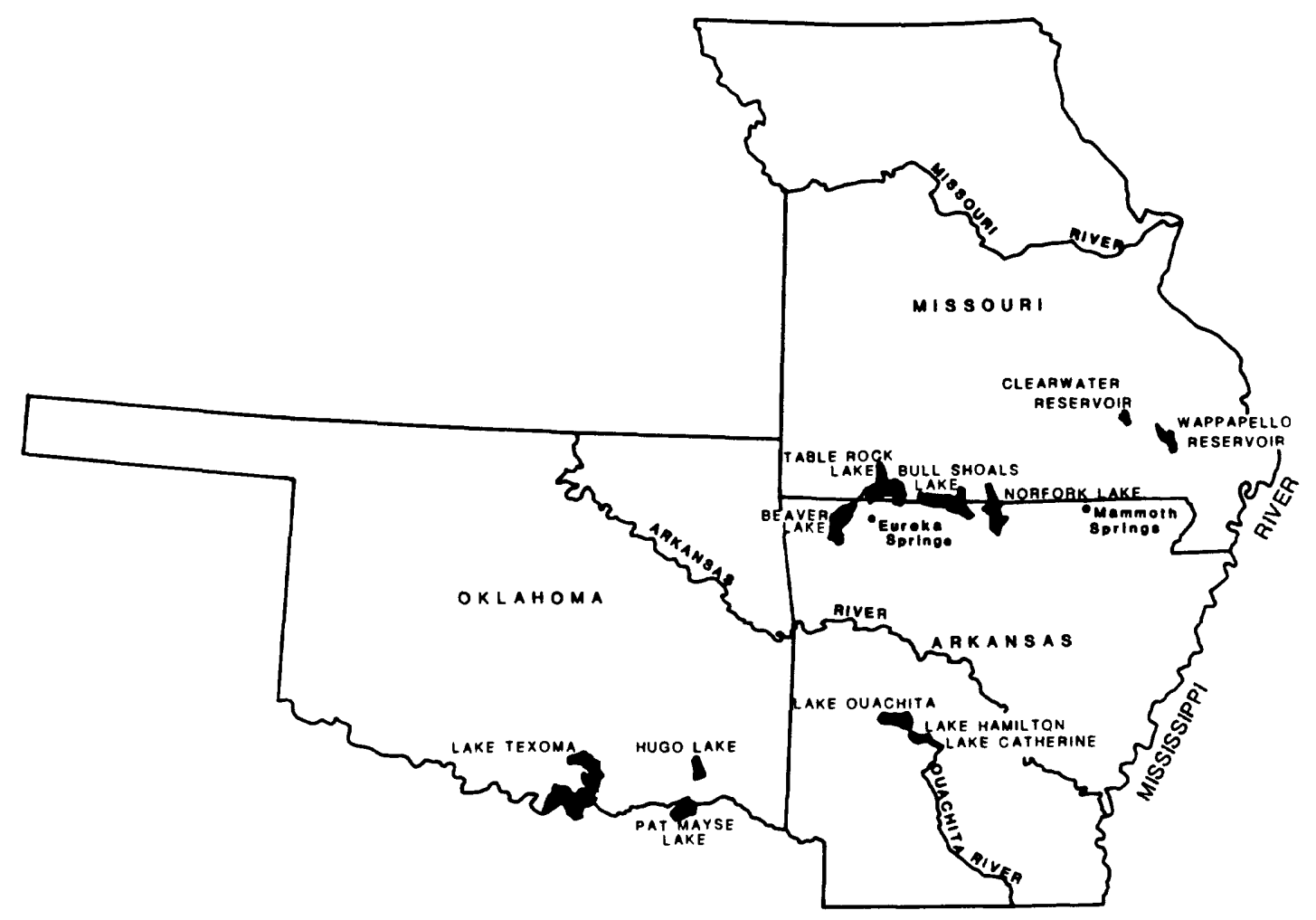

$\int_{0}^{0} \frac{50100}{100} 200$ KILOMETERS

Figure 1.--Location of study area. 
GENERAL SETTING

Arkansas has a diverse topography. The State is located in the Ozark Plateaus, Ouachita, and Coastal Plain physiographic provinces (Fenneman, 1938) (fig. 2). The Ozark and Ouachita Mountain ranges have an elevation as high as 2,700 feet ( $f t$ ) above sea level. Streams in the Ozark Plateaus and the southern half of the Ouachita Mountains tend to have sustained flows during dry seasons, whereas, streams in the Arkansas Valley (fig. 2) and the northern half of the Ouachita Mountains generally go dry (Hunrichs, 1983). The Mississippi Alluvial Plain and the West Gulf Coastal Plain (fig. 2) compose the southeastern part of the State; this is primarily an agricultural area and is relatively flat, with elevations that range from 55 to $500 \mathrm{ft}$ above sea level. The higher parts of the state are used mainly for raising cattle and poultry.

Arkansas has many springs, especially in the foothills of the Ouachita and Ozark Mountains. Thousands of people bath in the water from Arkansas springs each year for therapeutic reasons. The Eureka Springs area in the Ozarks contains approximately 65 springs. Mammoth Spring in the Ozarks is one of the largest springs in Arkansas, with an average discharge of 203 million gallons per day (Mgal/d). In the Hot Springs area in the Ouachitas, 51 springs yield about $1 \mathrm{Mgal} / \mathrm{d}$.

Arkansas' climate is mild and moderately humid. Average annual precipitation ranges from about 40 to 58 inches (in.) (fig. 3). Monthly precipitation exhibits a pronounced seasonal pattern; May usually has the most precipitation and January and October the least. Runoff ranges from about 12 to 32 in. per year, depending on the precipitation pattern (Freiwald, 1985).

Average annual evaporation from shallow lakes ranges from about 36 in. in the northeast to about $44 \mathrm{in}$. in the southwest (Farnsworth and others, 1982). 


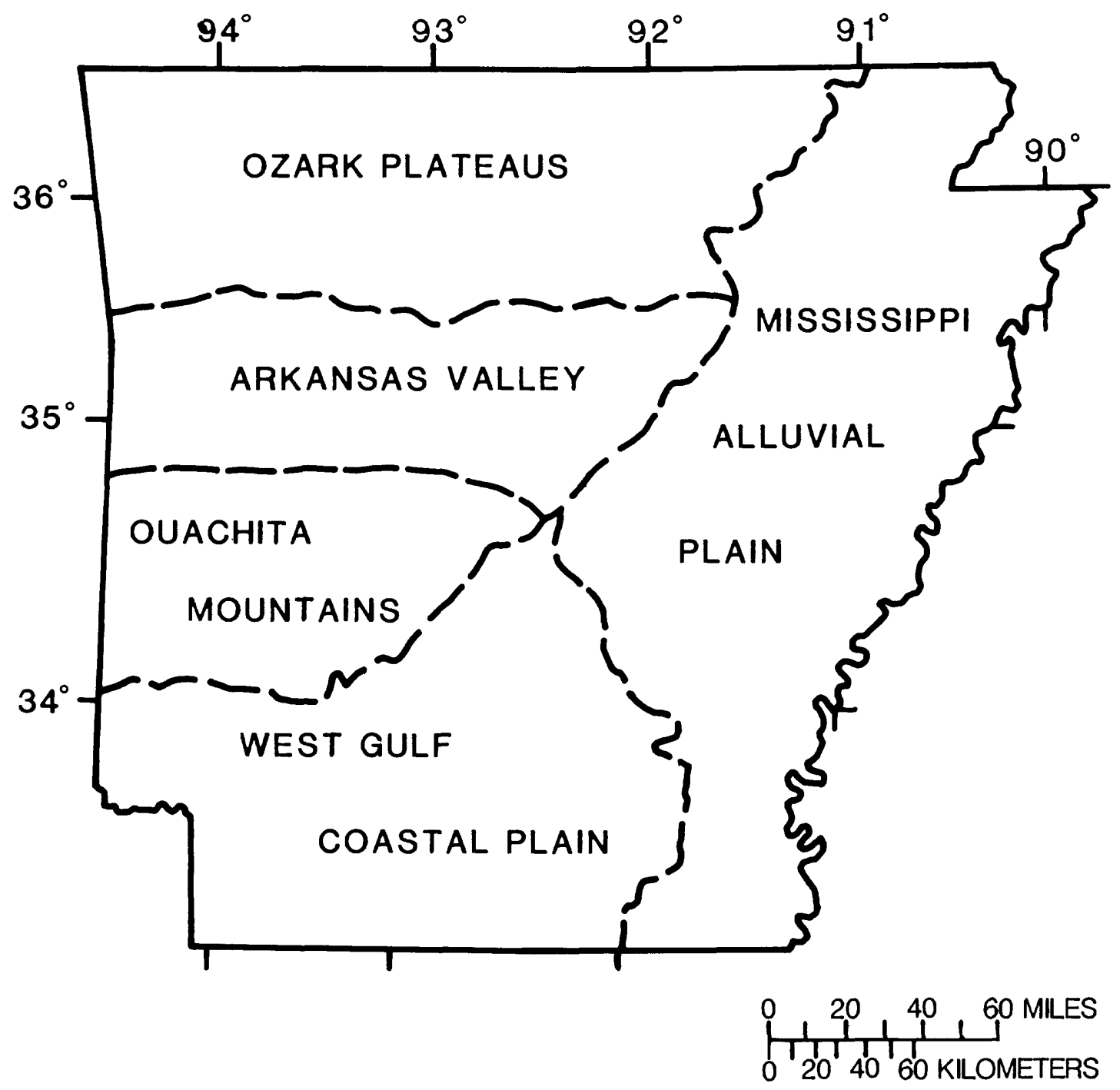

Figure 2.--Physiographic provinces in Arkansas. 


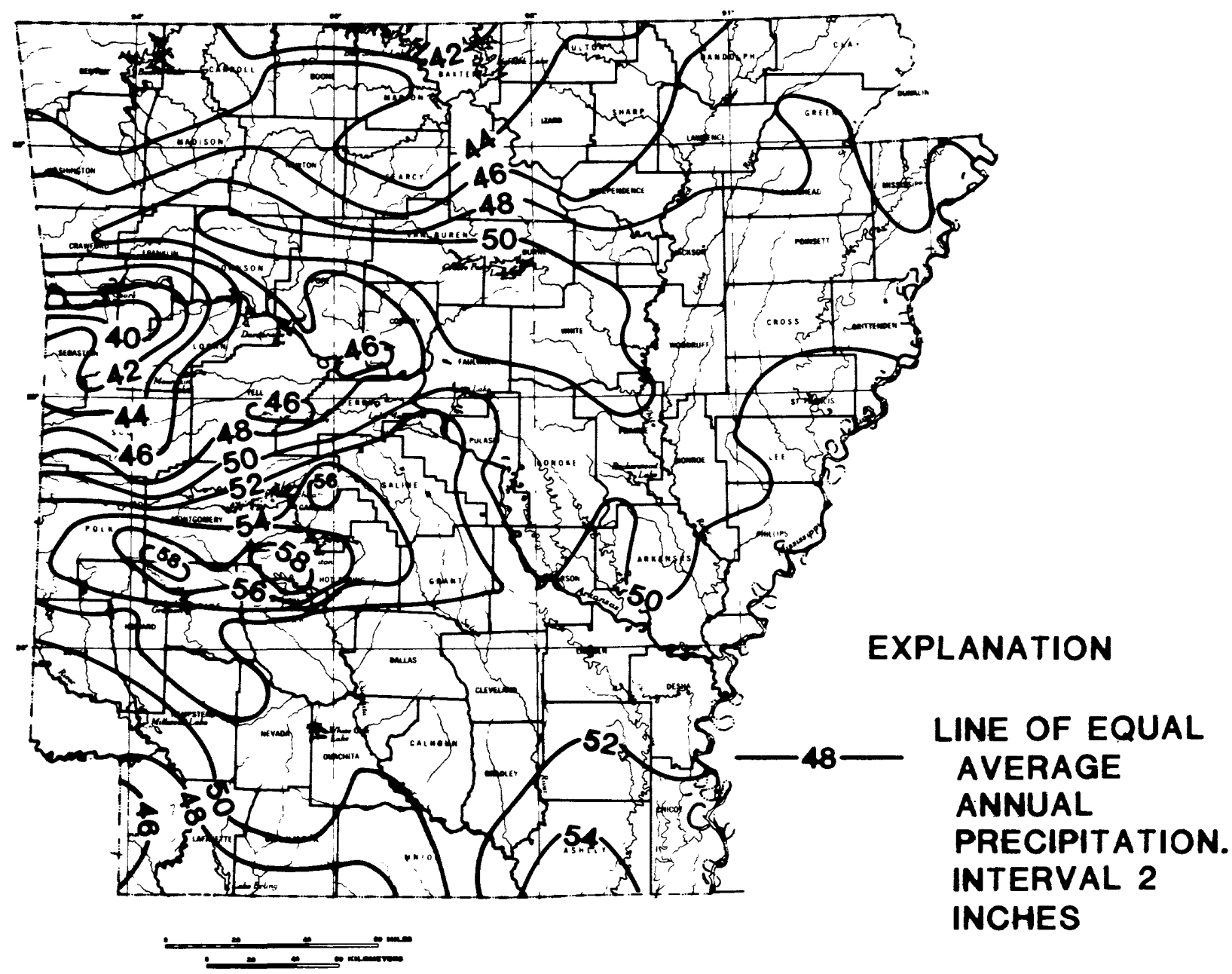

Figure 3.--Mean annual precipitation for Arkansas for the base period 1951-80 (from Freiwald, 19.85). 
Peak data for 254 gaging stations that have 10 or more years of record were used in preparing this report. Of these stations, 3 are in Missouri, 3 in Oklahoma, and 12 in Louisiana.

The flood data used in this report were, for the most part, collected by the U.S. Geological Survey and the U.S. Army Corps of Engineers. The flood data have been collected at two types of gaging stations: (1) regular gaging stations where records of daily stage and discharge are collected, and (2) crest-stage gaging stations where only the maximum peak stage and discharge for each year are determined. Of the 254 gaging stations used, 160 are regular gaging stations and 94 are crest-stage gaging stations. Generally, the regular gaging stations are on large streams and have been operated for relatively long periods. Only a small percentage of the drainage areas upstream from these gaging stations are less than $100 \mathrm{mi}^{2}$. The crest-stage gaging program was begun in 1960 to fill the need for flood data on small areas. The drainage areas upstream from these sites range from less than $0.1 \mathrm{mi}^{2}$ to about $50 \mathrm{mi}^{2}$.

The flood-frequency analysis presented in this report for streams with drainage areas of less than $3,000 \mathrm{mi}^{2}$ is based on 200 sites with at least 10 years of peak discharge data collected through 1984 and free of significant regulation. Sixty-five gaging stations are on regulated streams or on streams with drainage areas greater than $3,000 \mathrm{mi}^{2}$. At 11 of the 65 stations, data that were collected prior to regulation were used in the flood-frequency analysis. Figure 4 is a map of Arkansas showing the location of all gaging stations used.

A summary of the distribution of data and average length of record for each station is as follows:

\begin{tabular}{lcc}
$\begin{array}{c}\text { Drainage area, } \\
\text { in square miles }\end{array}$ & $\begin{array}{c}\text { Number } \\
\text { of } \\
\text { stations }\end{array}$ & $\begin{array}{r}\text { Average length of } \\
\text { record, in years }\end{array}$ \\
Less than 1 & 37 & 22 \\
1 to 5 & 39 & 22 \\
5 to 10 & 13 & 21 \\
10 to 50 & 21 & 23 \\
50 to 100 & 9 & 29 \\
100 to 500 & 51 & 32 \\
500 to 1,000 & 11 & 39 \\
1,000 to 2,000 & 16 & 51 \\
2,000 to 3,000 & 3 & \\
\hline
\end{tabular}




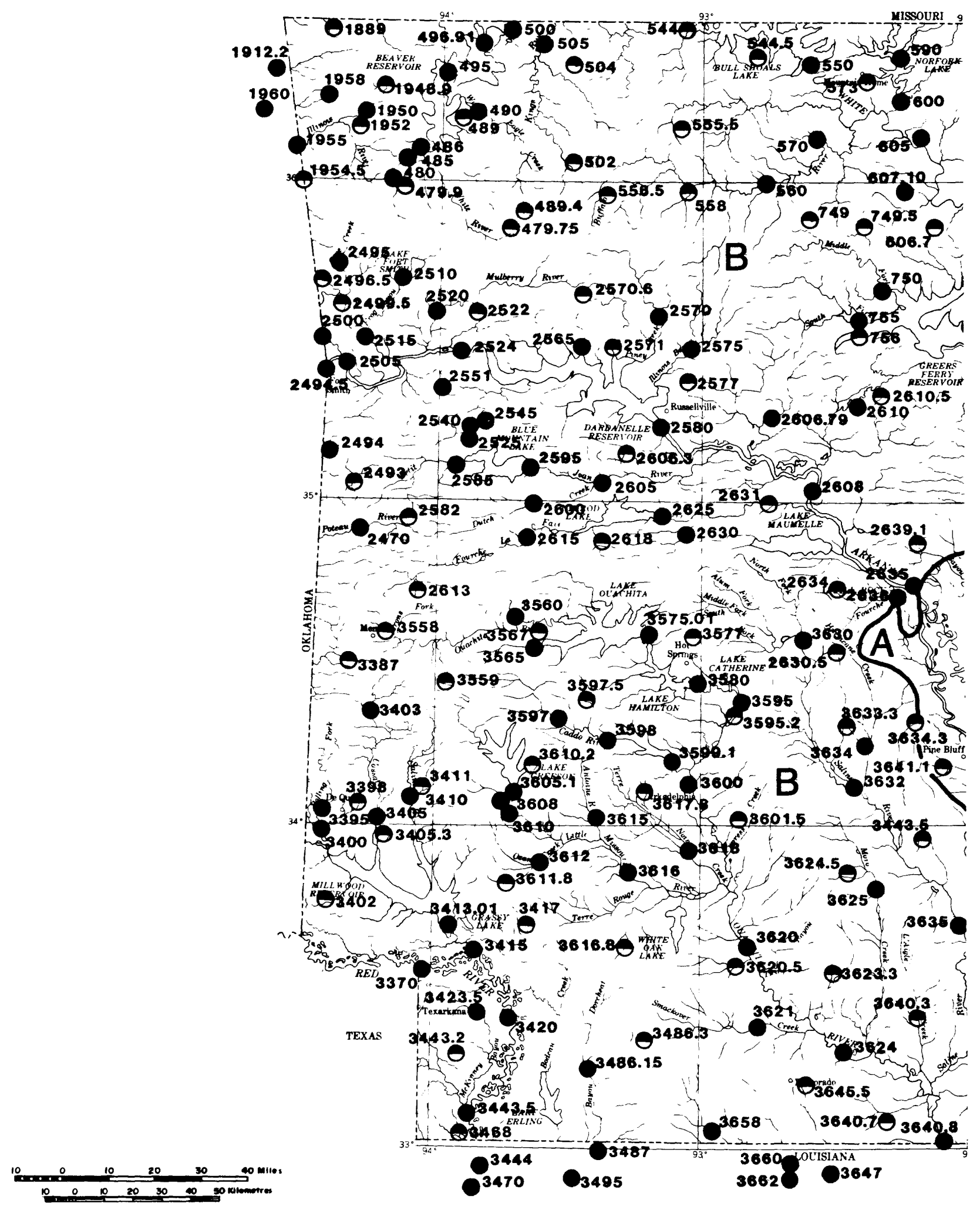

Figure 4.--Regional boundaries and 


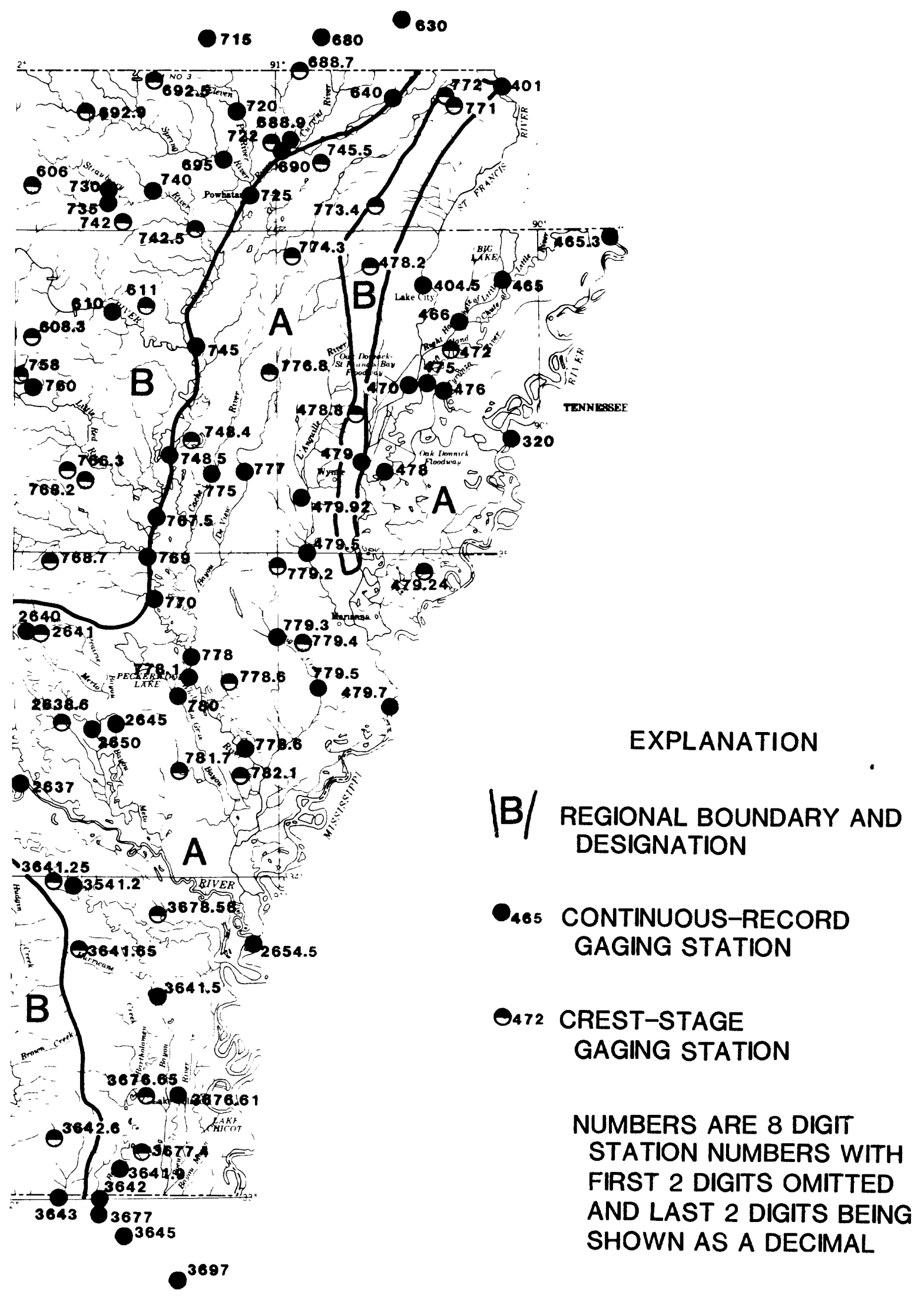

locations of gaging stations. 
A flood-frequency curve is the relation of flood-peak magnitude to probability of exceedance or recurrence interval. Probability of exceedance is the chance of a given flood magnitude being exceeded in any one year. A 5-year flood for example has the probability of 0.2 (or 20 percent chance) of being exceeded in any given year. Recurrence interval is the reciprocal of probability of exceedance times 100 and is the average number of years between exceedances for a long period of record. A 5-year flood may be expected to be exceeded on the average of once in 5 years or, 20 times in 100 years. This does not mean floods occur at uniformly spaced intervals. In fact, a flood of this magnitude can be exceeded more than once in the same year, or can occur in consecutive years.

The flood-frequency relation for a stream where gaging-station data are available can be defined by fitting the array of annual peak discharges (1argest instantaneous discharge for each year) to a theoretical distribution. The Hydrology Subcommittee of the Interagency Advisory Committee on Water Data (1982) has recommended a uniform technique for determining floodflow frequencies by fitting the logarithms of the annual peak discharges to a Pearson Type III distribution and has described these calculations in detail. This procedure is now generally accepted by most Federal and state agencies and is referred to as the log-Pearson Type III frequency distribution. Annual peak discharges for each gaging station used in this study were fitted to the log-Pearson Type III distribution. Peak discharges for recurrence intervals of $2,5,10,25,50$ and 100 years were computed for each station and are 1isted in tables 1, 2, and 3. For those stations where regulation began during the data collection period, discharge values are presented for both the unregulated and the regulated period.

\section{FLOOD MAGNITUDE AND FREQUENCY AT UNGAGED SITES ON STREAMS DRAINING LESS THAN 3,000 SQUARE MILES}

Flood-frequency relations can be estimated for ungaged sites up to 3,000 $\mathrm{mi}^{2}$ through the use of the equations presented in this section. The equations were developed by relating the 2-, 5-, 10-, 25-, 50-, and 100-year floods to basin and climatic characteristics. Although numerous basin and climatic characteristics were investigated, the ones that were most significant for this study were drainage area, main channel slope, main channel length, mean annual precipitation, and mean basin elevation. The basin and climatic characteristic, equation development, and accuracy and limitations of the results are described in the following paragraphs. 
Table 1.-Discharge, for selected recurrence interval, at gaging stations affecting Region $A$

IA, drainage area; S, slope; L, length; YRS, years of record. Numbers on line with station name are based on station data. Numbers on line below station name are values computed by weighting the regression discharge with station discharge.]

\begin{tabular}{|c|c|c|c|c|c|c|c|c|c|c|c|}
\hline \multirow{2}{*}{$\begin{array}{l}\text { Station } \\
\text { 1dentifi- } \\
\text { cation } \\
\text { number } \\
\end{array}$} & \multirow[b]{2}{*}{ Station name } & \multirow[b]{2}{*}{ A } & \multirow[b]{2}{*}{$\mathbf{s}$} & \multirow[b]{2}{*}{ L } & \multirow[b]{2}{*}{ YRS } & \multicolumn{6}{|c|}{$\begin{array}{l}\text { Discharge (cubic feet per second), } \\
\text { for recurrence interval (years) }\end{array}$} \\
\hline & & & & & & 2 & 5 & 10 & & 50 & 100 \\
\hline \multirow[t]{2}{*}{7047200} & Ditch no. 45 near Lepanto, Ark. & 2.16 & 1.2 & 3.3 & 23 & 170 & 198 & 213 & 230 & 240 & 250 \\
\hline & & & & & & 167 & 197 & 216 & 237 & 250 & 263 \\
\hline \multirow[t]{2}{*}{7047600} & Tyronza River near Tyronza, Ark. & 290 & 0.7 & 55.0 & 36 & 4,170 & 5,030 & 5,530 & 6,100 & 6,480 & 6,850 \\
\hline & & & & & & 4,170 & 4,910 & 5,420 & 6,030 & 6,440 & 6,830 \\
\hline \multirow[t]{2}{*}{7047924} & Crooked Bayou tributary at State & .48 & 4.2 & 1.2 & 20 & 110 & 204 & 281 & 395 & 492 & 599 \\
\hline & Highway 149 , at Hughes, Ark. & & & & & 106 & 190 & 255 & $5 \quad 353$ & $53 \quad 436$ & 526 \\
\hline 7047942 & L'Anguille River near Colt, Ark. & 535 & 0.9 & 58.7 & 14 & $\begin{array}{l}5,900 \\
5,730\end{array}$ & $\begin{array}{l}9,320 \\
8,800\end{array}$ & $\begin{array}{l}11,600 \\
10,800\end{array}$ & $\begin{array}{l}14,400 \\
13,300\end{array}$ & $\begin{array}{ll}\text { do } & 16,400 \\
\text { jo } & 15,200\end{array}$ & $\begin{array}{l}18,400 \\
16,900\end{array}$ \\
\hline $7063000^{\mathbf{a}}$ & Black River at Poplar Bluff, Mo. 1 & 1,245 & 6.2 & 115.0 & 25 & $\begin{array}{l}16,100 \\
15,900\end{array}$ & $\begin{array}{l}34,300 \\
32,700\end{array}$ & $\begin{array}{l}49,800 \\
46,500\end{array}$ & $\begin{array}{l}73,000 \\
67,600\end{array}$ & $\begin{array}{l}0,92,700 \\
\text { Do } 85,400\end{array}$ & $\begin{array}{l}114,000 \\
105,000\end{array}$ \\
\hline $7064000^{a}$ & Black River near Corning, Ark. & 1,749 & 3.6 & 181.0 & 32 & $\begin{array}{l}12,200 \\
12,300\end{array}$ & $\begin{array}{l}21,300 \\
21,300\end{array}$ & $\begin{array}{l}28,100 \\
28,000\end{array}$ & $\begin{array}{l}37,300 \\
37,100\end{array}$ & $\begin{array}{l}\text { Do } 44,500 \\
\text { on } 44,100\end{array}$ & $\begin{array}{l}51,900 \\
51,400\end{array}$ \\
\hline 7074550 & Village Creek near $0^{\prime}$ Kean, Ark. & 6.24 & 1.8 & 4.8 & 21 & 219 & 498 & 764 & 1,200 & on 1,610 & 2,090 \\
\hline & & & & & & 235 & 498 & 732 & 1,110 & 1,460 & 1,860 \\
\hline 7074855 & Cypress Creek tributary near & 5.54 & 3.3 & 6.0 & 20 & 316 & 484 & 604 & 764 & 888 & 1,020 \\
\hline & Augusta, Ark. & & & & & 320 & 488 & 608 & 766 & 887 & 1,010 \\
\hline 7077380 & Cache R1ver at Egypt, Ark. & 701 & 1.0 & 72.9 & 35 & 4,390 & 5,870 & 6,870 & 8,160 & 9,140 & 10,100 \\
\hline & & & & & & 4,510 & 6,130 & 7,300 & 8,710 & 9,770 & 10,800 \\
\hline 7077430 & Willow Ditch near Egypt, Ark. & .48 & 4.0 & 1.2 & 22 & 36 & 67 & 95 & 139 & 179 & 225 \\
\hline & & & & & & 41 & 75 & 104 & 147 & 184 & 226 \\
\hline 7077500 & Cache River at Patterson, Ark. & 1,037 & 0.8 & 138.0 & 52 & 6,540 & 9,520 & 11,600 & 14,300 & 0016,500 & 18,700 \\
\hline & & & & & & 6,530 & 9,460 & 11,500 & 14,200 & 0016,300 & 18,400 \\
\hline 7077680 & Threemile Creek near Amagon, Ark. & 7.93 & 1.3 & 7.4 & 20 & 295 & 394 & 448 & 506 & $06 \quad 542$ & 574 \\
\hline & & & & & & 300 & 405 & 467 & 535 & 578 & 617 \\
\hline 7077700 & Bayou Deview at Morton, Ark. & 421 & 2.3 & 70.4 & 43 & 3,210 & 4,270 & 4,920 & 5,670 & 6,200 & 6,700 \\
\hline & & & & & & 3,330 & 4,550 & 5,400 & 6,330 & 6,990 & $7,63 n$ \\
\hline 7077860 & Boat Gunwale Slaah tributary near & 10.0 & 1.9 & 7.2 & 22 & 340 & 471 & 545 & 625 & 677 & 723 \\
\hline & Holly Grove, Ark. & & & & & 353 & 498 & 589 & 686 & 751 & 810 \\
\hline 7077920 & Big Creek at Goodwin, Ark. & 31.1 & 0.9 & 17.4 & 24 & 563 & 758 & 869 & 992 & 1,070 & 1,140 \\
\hline & & & & & & 579 & 785 & 913 & 1,050 & 1,140 & $1,22 n$ \\
\hline 7077940 & Spring Creek near Aubrey, Ark. & 38.0 & 1.5 & 11.0 & 20 & $\begin{array}{l}1,360 \\
1,330\end{array}$ & $\begin{array}{l}1,840 \\
1,800\end{array}$ & $\begin{array}{l}2,110 \\
2,080\end{array}$ & $\begin{array}{l}2,400 \\
2,400\end{array}$ & $\begin{array}{l}2,590 \\
2,610\end{array}$ & $\begin{array}{l}2,760 \\
2,810\end{array}$ \\
\hline 7077950 & Big Creek at Poplar Grove, Ark. & 448 & 0.7 & 67.3 & 14 & 3,010 & 4,700 & 5,760 & 7,010 & 7,880 & 8,690 \\
\hline & & & & & & 3,160 & 4,830 & 5,910 & 7,160 & 8,030 & $8,83 n$ \\
\hline 7078000 & LaGrue Bayou near Stuttgart, Ark. & 175 & 0.8 & 44.8 & 19 & $\begin{array}{l}2,400 \\
2,360\end{array}$ & $\begin{array}{l}3,990 \\
3,790\end{array}$ & $\begin{array}{l}5,070 \\
4,730\end{array}$ & $\begin{array}{l}6,440 \\
5,950\end{array}$ & $\begin{array}{l}7,440 \\
6,830\end{array}$ & $\begin{array}{l}8,420 \\
7,700\end{array}$ \\
\hline 7078170 & Little LaGrue Bayou tributary near & 1.51 & 3.3 & 4.3 & 20 & 184 & 221 & 239 & 258 & 269 & 279 \\
\hline & Dowstet Art & & & & & 177 & 215 & 236 & $26 n$ & 276 & 291 \\
\hline 7078210 & Tarleton Creek tributary at & .20 & 18.3 & 1.0 & 22 & 70 & 117 & 156 & 215 & 267 & 326 \\
\hline & & & & & & 69 & 115 & 152 & 208 & 256 & 31 \\
\hline 7263860 & Mile Branch near Tomberlin, Ark. & 2.75 & 3.5 & 3.1 & 17 & 363 & 502 & 580 & 665 & 720 & 770 \\
\hline & & & & & & 345 & 476 & 550 & 641 & 702 & 758 \\
\hline 7264000 & Bayou Meto near Lonoke, Ark. & 207 & 1.3 & 52.4 & 38 & 2,140 & 3,010 & 3,580 & 4,310 & 4,860 & 5,400 \\
\hline & & & & & & 2,180 & 3,080 & 3,690 & 450 & 5,020 & 5,580 \\
\hline 7264100 & White Oak Branch near Lonoke, & 8.41 & 5.2 & 4.4 & 24 & 864 & 1,270 & 1,520 & 1,790 & 1,970 & 2,130 \\
\hline & & & & & & 836 & 1,230 & 1,470 & 1,760 & 1,950 & 2,130 \\
\hline 7364110 & Nevins Creek tributary near & .75 & 36.6 & 1.3 & 24 & 142 & 264 & 364 & 512 & 638 & 776 \\
\hline & Pine Bluff, Ark. & & & & & 150 & 280 & 388 & 544 & 675 & 819 \\
\hline 7364120 & Bayou Bartholomew near Star & 215 & 0.6 & 81.7 & 32 & 1,720 & 2,420 & 2,840 & 3,320 & 3,650 & 3,950 \\
\hline & & & & & & & 2,420 & 2,840 & 3,310 & 3,640 & 3,940 \\
\hline 7364125 & Cane Creek at Star City, Ark. & 4.91 & 40.8 & 3.0 & 22 & 1, & & 1,860 & 2,240 & & 2,730 \\
\hline & & & & & & & 1,530 & 1,890 & 2,360 & 2,690 & 3,030 \\
\hline 7364150 & Bayou Bartholomew near & 576 & 0.5 & 167.0 & 47 & 3,180 & 4,360 & 5,060 & 5,860 & 6,390 & 6,890 \\
\hline 7364165 & $\begin{array}{l}\text { McGehee, Ark. } \\
\text { Upper Cutoff Creek near }\end{array}$ & 18.0 & 11.0 & 7.7 & 21 & $\begin{array}{r}3,180 \\
899\end{array}$ & $\begin{array}{l}4,340 \\
1,610\end{array}$ & $\begin{array}{l}5,040 \\
2,220\end{array}$ & $\begin{array}{l}3,840 \\
3,160\end{array}$ & $\begin{array}{l}6,310 \\
4,000\end{array}$ & $\begin{array}{l}6,860 \\
4,960\end{array}$ \\
\hline & Monticello, Ark. & & & & & 933 & 1,670 & 2,300 & 3,230 & 4,040 & 4,950 \\
\hline 7364190 & Bayou Bartholomew at & 1,170 & 0.4 & 269.0 & 48 & 4,750 & 5,980 & 6,700 & 7,530 & 8,110 & 8,650 \\
\hline 7364200 & Wilmot, Ark. & & & & & & 5,970 & 6,720 & & 8,160 & 8,710 \\
\hline & $\begin{array}{l}\text { Bayou Bartholomew nea } \\
\text { Jones, La. }\end{array}$ & & & & 27 & $\begin{array}{l}4,370 \\
4,400\end{array}$ & $\begin{array}{l}5,920 \\
5,910\end{array}$ & $\begin{array}{l}6,800 \\
6,800\end{array}$ & $\begin{array}{l}7,760 \\
7,780\end{array}$ & $\begin{array}{l}8,390 \\
8,420\end{array}$ & $\begin{array}{l}8,960 \\
9,000\end{array}$ \\
\hline 7364500 & Bayou Bartholomew near & 1,645 & 0.4 & 318.0 & 53 & 7,040 & 9,040 & 10,300 & 11,700 & 12,700 & 13,700 \\
\hline & & & & & & & 8,920 & 10,100 & 11,500 & 012,500 & 13,500 \\
\hline 7367658 & Cypress Creek Canal No. 19 & .94 & 3.6 & 1.6 & 24 & 155 & $20 \kappa$ & 239 & 280 & $30 \quad 310$ & 340 \\
\hline & & & & & & 152 & 203 & 237 & 281 & 312 & 344 \\
\hline 7367740 & Camp Bayou near Parkdale, Ark. & 1.86 & 4.6 & 2.6 & 22 & 230 & 296 & 335 & 379 & 409 & 436 \\
\hline & & & & & & 226 & 297 & 342 & 395 & 433 & 468 \\
\hline
\end{tabular}

a Before regulation; regulated values are shown in table 3 . 
Table 2.-Discharge, for selected recurrence intervals, at gaging stations affecting Region B

[A, dralnage area; S, slope; P, precipltation; E, elevation, YRS, years of record. Numbers on line with station name are based on station data. Numbers on line below station name are values computed by welghting the regression discharge with the station discharge.]

\begin{tabular}{|c|c|c|c|c|c|c|c|c|c|c|c|c|}
\hline \multirow{2}{*}{$\begin{array}{l}\text { Station } \\
\text { 1dentif } 1- \\
\text { cation } \\
\text { number }\end{array}$} & & & & & & & & $\begin{array}{l}\text { Ischarg } \\
\text { for reci }\end{array}$ & $\begin{array}{l}\text { e (cubic } \\
\text { urrence }\end{array}$ & $\begin{array}{l}\text { feet per } \\
\text { Interval }\end{array}$ & $\begin{array}{r}\text { r second } \\
\text { (years) }\end{array}$ & \\
\hline & Station name & A & $s$ & $\mathrm{P}$ & E & YRS & 2 & 5 & 10 & 25 & 50 & 100 \\
\hline 7047820 & Murray Creek near Jonesboro, & 1.38 & 33.2 & 48 & 320 & 24 & 503 & 802 & 1,020 & 1,300 & 1,520 & 1,750 \\
\hline & Ark. & & & & & & 465 & 728 & 926 & 1,180 & 1,420 & 1,670 \\
\hline 7047880 & Pope Creek tributary at & .08 & 200.0 & 49 & 370 & 22 & 52 & 93 & 129 & 185 & 236 & 296 \\
\hline & Birdeye, Ark. & & & & & & 48 & 84 & 115 & 157 & 199 & 248 \\
\hline 7047975 & Dog Branch at St. Paul, Ark. & 1.23 & 271.0 & 48 & 1,990 & 21 & 204 & 395 & 545 & 755 & 924 & 1,100 \\
\hline & & & & & & & 220 & 441 & 638 & 892 & 1,120 & 1,350 \\
\hline 7047990 & West Fork White River tribu- & .67 & 290.0 & 44 & 1,520 & 25 & 172 & 400 & 605 & 919 & 1,190 & 1,490 \\
\hline & tary near Greenland, Ark. & & & & & & 171 & 383 & 567 & 821 & 1,060 & 1,310 \\
\hline 7048000 & West Fork White RIver at & 83.1 & 27.5 & 45 & 1,710 & 38 & 8,740 & 16,500 & 22,900 & 32,000 & 39,600 & 47,800 \\
\hline & Greenland, Ark. & & & & & & 8,590 & 16,100 & 22,300 & 31,000 & 38,500 & 46,600 \\
\hline 7048600 & White River near & 400 & 14.4 & 45 & 1,600 & 21 & 21,900 & 38,300 & 50,500 & 66,900 & 79,700 & 92,800 \\
\hline & Fayetteville, Ark. & & & & & & 21,400 & 37,300 & 50),, 000 & 67,600 & 82,400 & 97,400 \\
\hline 7048900 & Whitener Branch $t r$ butary & 1.07 & 105.0 & 43 & 1,380 & 23 & 155 & $3 \quad 312$ & 460 & 706 & 940 & 1,220 \\
\hline & near Spring Valley, Ark. & & & & & & 165 & 344 & 521 & 774 & 1,020 & 1,290 \\
\hline 7048940 & War Eagle Creek near & 22.4 & 42.6 & 48 & 1,920 & 22 & 3,020 & 5,990 & 8,460 & 12,100 & 15,200 & 18,600 \\
\hline & Witter, Ark. & & & & & & 3,010 & 5,890 & 8,330 & 11,700 & 14,700 & 18,000 \\
\hline 7049000 & War Eagle Creek near & 262 & 8.5 & 45 & 1,590 & 25 & 12,600 & 22,400 & 29,600 & 39,100 & 46,300 & 53,600 \\
\hline & Hindsullle, Ark. & & & & & & 12,300 & 21,700 & 28,800 & 38,400 & 46,300 & 54,300 \\
\hline 7049500 & White Rlver near Rogers, & 1,020 & 5.6 & 44 & 1,500 & 13 & 24,200 & 45,000 & 60,400 & 80,900 & 96,500 & 112,000 \\
\hline & Ark. & & & & & & 24,400 & 44,700 & 60,700 & 83,200 & 101,000 & 120,000 \\
\hline 7050000 & White River at Beaver, & 1,238 & 3.8 & 44 & 1,450 & 39 & 30,200 & 49,100 & 63,500 & 83,500 & 99,700 & 117,000 \\
\hline & Ark. & & & & & & 29,700 & 48,100 & 62,500 & 83,000 & 99,800 & 118,000 \\
\hline 7050200 & Maxwell Creek at Kingston, & 2.75 & 141.0 & 45 & 1,627 & 21 & 612 & 1,310 & 1,900 & 2,800 & 3,570 & 4,410 \\
\hline & Ark. & & & & & & 596 & 1,230 & 1,760 & 2,490 & 3,160 & 3,880 \\
\hline 7050400 & Freeman Branch at & .73 & 129.0 & 43 & 1,361 & 20 & 194 & 324 & 419 & 547 & 646 & 749 \\
\hline & Berryville, Ark. & & & & & & 189 & 326 & 443 & 592 & 731 & 868 \\
\hline 7050500 & K1ngs R1ver near & 527 & 6.8 & 43 & 1,540 & 46 & 18,100 & 32,400 & 42,800 & 56,700 & 67,300 & 78,100 \\
\hline & Berryville, Ark. & & & & & & 17,900 & 31,800 & 42,200 & 56,300 & 67,600 & 79,100 \\
\hline 7054400 & Charley Creek near Omaha, & 3.41 & 112.0 & 42 & 1,040 & 21 & 1,160 & 2,000 & 2,690 & 3,710 & 4,590 & 5,560 \\
\hline & Ark. & & & & & & 1,060 & 1,770 & 2,330 & 3,130 & 3,880 & 4,710 \\
\hline 7054450 & East Sugarloaf Creek tribu- & .85 & 216.0 & 42 & 1,060 & 23 & 256 & 500 & 720 & 1,070 & 1,400 & 1,780 \\
\hline & tary near Lead Hill, Ark. & & & & & & 245 & 468 & 664 & 946 & 1,230 & 1,540 \\
\hline 7055550 & Crooked Creek tributary near & 4.36 & 56.1 & 45 & 1,278 & 24 & 616 & 1,130 & 1,570 & 2,240 & 2,830 & 3,500 \\
\hline & Dagpatch, Ark. & & & & & & 633 & 1,200 & 1,720 & 2,450 & 3,130 & 3,840 \\
\hline 7055650 & Smith Creek near Boxley, Ark & 8.35 & 137.0 & 47 & 1,905 & 21 & 1,450 & 3,330 & 5,000 & 7,550 & 9,730 & 12,100 \\
\hline & & & & & & & 1,430 & 3,130 & 4,560 & 6,560 & 8,370 & 10,300 \\
\hline 7055800 & Dry Branch near Vendor, Ark. & 6.15 & 233.0 & 45 & 1,511 & 21 & 1,100 & 2,290 & 3,300 & 4,830 & 6,140 & 7,580 \\
\hline & & & & & & & 1,080 & 2,290 & 3,120 & 4,410 & 5,600 & 6,880 \\
\hline 7056000 & Buffalo Rlver near St. Joe, & 829 & 10.4 & 45 & 1,490 & 46 & 36,000 & 66,700 & 90,000 & 122,000 & 147,000 & 173,000 \\
\hline & Ark. & & & & & & 35,400 & 64,700 & 87,000 & 118,000 & 143,000 & 169,000 \\
\hline 7057000 & Buffalo Rlver near Rush, & 1,096 & 7.1 & 44 & 1380 & 45 & 39,600 & 73,800 & 101,000 & 139,000 & 171,000 & 205,000 \\
\hline & Ark. & & & & & & 38,700 & 70,900 & 95,900 & 131,000 & 161,000 & 193,000 \\
\hline 7057300 & Dodd Creek tributary near & .76 & 118.0 & 43 & 850 & 24 & 261 & 425 & 540 & 687 & 798 & 909 \\
\hline & Mountaln Home, Ark. & & & & & & 248 & 3407 & 530 & 685 & 828 & 969 \\
\hline 7059000 & North Fork River near & 1,612 & 6.4 & 40 & 1300 & 15 & 24,800 & 42,100 & 53,900 & 68,700 & 79,400 & 89,900 \\
\hline & Henderson, Ark. & & & & & & 26,100 & 47,100 & 65,100 & 92,200 & 112,000 & 133,000 \\
\hline 7060600 & Band M111 Creek near & 1.25 & 85.3 & 44 & 737 & 24 & 238 & 428 & 595 & 861 & 1,100 & 1,390 \\
\hline & Brockwell, Ark. & & & & & & 242 & 450 & 646 & 923 & 1,190 & 1,480 \\
\hline 7060670 & Hughes Creek near Mountain & 3.20 & 209.0 & 47 & 850 & 21 & 857 & 1,310 & 1,650 & 2,100 & 2,460 & 2,830 \\
\hline & V1ew, Ark. & & & & & & 819 & 1,280 & 1,670 & 2,190 & 2,660 & 3,130 \\
\hline 7060710 & North Sylamore Creek near & 58.1 & 15.4 & 45 & 650 & 19 & 4,180 & 10,800 & 16,900 & 26,600 & 34,900 & 44,200 \\
\hline & Fifty Six, Ark. & & & & & & 4,190 & 10,000 & 14,900 & 22,100 & 28,400 & 35,500 \\
\hline 7060830 & Wolf Bayou near Drasco, & .27 & 97.8 & 49 & 1050 & 21 & 58 & 123 & 176 & 251 & 312 & 375 \\
\hline & Ark. & & & & & & 64 & 137 & 203 & 285 & 360 & 434 \\
\hline 7061100 & Gibbs Creek at Sulphur & 3.90 & 46.2 & 47 & 460 & 23 & 840 & 1,490 & 1,990 & 2,680 & 3,230 & 3,800 \\
\hline & Rock, Ark. & & & & & & 816 & 1,450 & 1,970 & 2,650 & 3,260 & 3,890 \\
\hline 7068000 & Current RIver at Donlphan, & 2,038 & 4.7 & 44 & 1000 & 66 & 26,000 & 48,300 & 65,800 & 90,200 & 110,000 & 131,000 \\
\hline & & & & & & & 26,800 & 50,300 & 69,800 & 97,500 & 120,000 & 143,000 \\
\hline 7068870 & Fourche River tributary & .19 & 161.0 & 46 & 455 & 21 & 140 & 202 & 245 & 302 & 346 & 392 \\
\hline & at Middlebrook, Ark. & & & & & & 127 & 181 & 222 & 275 & 326 & 380 \\
\hline 7068890 & Fourche River above & 229 & 10.8 & 46 & 510 & 15 & 15,600 & 28,800 & 38,800 & 52,400 & 63,000 & 73,800 \\
\hline & ahontas, Ark. & & & & & & 14,600 & 26,000 & 34,500 & 46,100 & & 66,300 \\
\hline 7069250 & Brush Creek near Mammoth & .48 & 156.0 & 44 & 685 & 24 & 296 & 525 & 705 & 963 & 1,170 & 1,400 \\
\hline & Spring, Ark. & & & & & & 271 & 462 & 604 & 795 & 969 & 1,160 \\
\hline 7069290 & Miller Creek near Salem, & 2.28 & 77.5 & 44 & 787 & 21 & 544 & 985 & 1,330 & 1,830 & 2,240 & 2,680 \\
\hline & & & & & & & 525 & 949 & & & & \\
\hline 7069500 & Spring River at Imboden, & 1,183 & 8.4 & 44 & 740 & 48 & 26,500 & 51,100 & 72,000 & 104,000 & 132,000 & 163,000 \\
\hline & & & & & & & & 52,200 & 74,100 & 107,000 & 136,000 & 166,000 \\
\hline 7071500 & Eleven Point Rlver near & 793 & 10.1 & 44 & 1000 & 63 & 9200 & 20,200 & 29,100 & 41,800 & 51,900 & 62,500 \\
\hline & Bardley, Mo. & & & & & & 10,200 & 23,000 & 34,400 & 51,000 & 64,200 & 77,400 \\
\hline 7072000 & Eleven Point River near & 1,134 & 10.2 & 43 & 850 & 53 & 12,000 & 23,200 & 33,600 & 50,600 & 66,500 & 85,600 \\
\hline & & & & & & & 13,500 & 27,700 & 42,200 & 65,100 & 85,300 & 107,000 \\
\hline 7072200 & Hubble Creek near & 1.33 & 50.6 & 47 & 470 & 24 & 616 & 840 & 980 & 1,150 & 1,270 & 1,380 \\
\hline & & & & & & & 570 & 780 & 938 & 1,140 & & 1,510 \\
\hline 7073000 & Strawberry River near & 217 & 6.1 & 44 & 740 & 41 & 9,130 & 14,800 & 19,000 & 24,600 & 29,000 & 33,600 \\
\hline & EvenIng Shade, Ark. & & & & & & & 14,600 & & & 29,900 & 35,000 \\
\hline 7073500 & P1ney Fork at Evening & 99.2 & 7.6 & 45 & 670 & 46 & 4,770 & 9,000 & 12,600 & 18,100 & 22,800 & 28,200 \\
\hline & Shade, Ark. & & & & & & 4,770 & 8,940 & 12,500 & 17,700 & 22,200 & 27,300 \\
\hline 7074000 & Strawberry River near & 473 & 6.0 & 45 & 680 & 48 & 14,800 & 27,800 & 38,900 & 55,800 & 70,700 & 87,600 \\
\hline & Poughkeepsie, Ark. & & & & & & 14,800 & 27,500 & 38,300 & 54,400 & 68,500 & 84,200 \\
\hline 7074200 & Dry Branch tributary near & 1.22 & 61.5 & 45 & 649 & 22 & 586 & 1,020 & 1,300 & 1,640 & 1,880 & 2,110 \\
\hline & S1dney, Ark. & & & & & & 538 & 902 & 1,140 & 1,420 & 1,670 & 1,920 \\
\hline
\end{tabular}


Table 2.-Discharge, for selected recurrence intervals, at gaging stations affecting Region B-Continued

\begin{tabular}{|c|c|c|c|c|c|c|c|c|c|c|c|c|}
\hline \multirow{2}{*}{$\begin{array}{l}\text { Station } \\
\text { Identifi- } \\
\text { cation } \\
\text { number }\end{array}$} & \multirow[b]{2}{*}{ Station name } & \multirow[b]{2}{*}{ A } & \multirow[b]{2}{*}{$\mathrm{s}$} & \multirow[b]{2}{*}{$P$} & \multirow[b]{2}{*}{ E } & \multirow[b]{2}{*}{ YRS } & \multicolumn{6}{|c|}{$\begin{array}{l}\text { D1scharge (cuble feet per second), } \\
\text { for recurrence interval (years) }\end{array}$} \\
\hline & & & & & & & 2 & 5 & 10 & 25 & 50 & 100 \\
\hline 7074250 & $\begin{array}{l}\text { Reeds Creek near Strawberry, } \\
\text { Ark. }\end{array}$ & 34.9 & 17.1 & 46 & 440 & 21 & $\begin{array}{l}2,980 \\
2,950\end{array}$ & $\begin{array}{l}5,770 \\
5,640\end{array}$ & $\begin{array}{l}8,180 \\
7,980\end{array}$ & $\begin{array}{l}11,900 \\
11,226\end{array}$ & $\begin{array}{l}15,100 \\
14,400\end{array}$ & $\begin{array}{l}18,800 \\
17,700\end{array}$ \\
\hline 7074900 & Trace Creek tributary near & .26 & 609.0 & 44 & 1,240 & 24 & 90 & 163 & 216 & 285 & 338 & 391 \\
\hline & Marshall, Ark. & & & & & & 88 & 161 & 218 & 292 & 355 & 419 \\
\hline 7074950 & Tick Creek near Leslie, & 1.58 & 116.0 & 45 & 1,519 & 23 & 307 & 652 & 937 & 1,350 & 1,680 & 2,040 \\
\hline 7075000 & $\begin{array}{l}\text { Middle Fork Little Red River } \\
\text { at Sh1rley, Ark. }\end{array}$ & 302 & 13.9 & 45 & 1,160 & 46 & $\begin{array}{l}22,600 \\
22,000\end{array}$ & $\begin{array}{l}43,700 \\
41,500\end{array}$ & $\begin{array}{l}61,700 \\
57,600\end{array}$ & $\begin{array}{l}89,1001 \\
80,746 !\end{array}$ & $\begin{array}{l}113,000 \\
102,000\end{array}$ & $\begin{array}{l}140,000 \\
126,000\end{array}$ \\
\hline 7075300 & $\begin{array}{l}\text { South Fork Little Red River } \\
\text { at Clinton, Ark. }\end{array}$ & 148 & 21.1 & 50 & 1,150 & 23 & $\begin{array}{l}10,900 \\
11,000\end{array}$ & $\begin{array}{l}21,900 \\
21,800\end{array}$ & $\begin{array}{l}30,900 \\
30,800\end{array}$ & $\begin{array}{l}43,900 \\
42,544\end{array}$ & $\begin{array}{l}54,600 \\
54,000\end{array}$ & $\begin{array}{l}66,200 \\
65,300\end{array}$ \\
\hline 7075500 & $\begin{array}{l}\text { South Fork tittle Red River } \\
\text { near Clinton, Ark. }\end{array}$ & 316 & 16.6 & 50 & 1,150 & 23 & $\begin{array}{l}22,200 \\
21,700\end{array}$ & $\begin{array}{l}34,800 \\
34,800\end{array}$ & $\begin{array}{l}43,200 \\
44,900\end{array}$ & $\begin{array}{l}53,800 \\
58,800\end{array}$ & $\begin{array}{l}61,400 \\
70,100\end{array}$ & $\begin{array}{l}69,000 \\
81,300\end{array}$ \\
\hline 7075600 & $\begin{array}{l}\text { Choctaw Creek tributary } \\
\text { near Choctaw, Ark. }\end{array}$ & 1.36 & 103.0 & 50 & 683 & 21 & $\begin{array}{l}239 \\
256\end{array}$ & $\begin{array}{l}448 \\
499\end{array}$ & $\begin{array}{l}633 \\
732\end{array}$ & $\begin{array}{r}927 \\
1,050\end{array}$ & $\begin{array}{l}1,200 \\
1,330\end{array}$ & $\begin{array}{l}1,510 \\
1,670\end{array}$ \\
\hline 7075800 & $\begin{array}{l}\text { D11l Branch tributary near } \\
\text { Ida, Ark. }\end{array}$ & .26 & 198.0 & 50 & 828 & 21 & $\begin{array}{l}58 \\
64\end{array}$ & $\begin{array}{l}124 \\
138\end{array}$ & $\begin{array}{l}180 \\
205\end{array}$ & $\begin{array}{l}264 \\
292\end{array}$ & $\begin{array}{l}335 \\
369\end{array}$ & $\begin{array}{l}412 \\
453\end{array}$ \\
\hline $707600 n^{a}$ & $\begin{array}{l}\text { Litile Red River near } \\
\text { Heber Springs, Ark. }\end{array}$ & 1,153 & 10.0 & 48 & 1,040 & 34 & $\begin{array}{l}54,500 \\
53,000\end{array}$ & $\begin{array}{l}74,400 \\
73,800\end{array}$ & $\begin{array}{l}86,400 \\
89,300\end{array}$ & $\begin{array}{ll}100,000 & 1 \\
111,000 & 1\end{array}$ & $\begin{array}{l}110,000 \\
123,215\end{array}$ & $\begin{array}{l}11,9000 \\
144,000\end{array}$ \\
\hline 7076630 & $\begin{array}{l}\text { Key Branch near Searcy, } \\
\text { Ark. }\end{array}$ & .66 & 84.8 & 50 & 332 & 22 & $\begin{array}{l}286 \\
264\end{array}$ & $\begin{array}{l}393 \\
367\end{array}$ & $\begin{array}{l}458 \\
446\end{array}$ & $\begin{array}{l}534 \\
546\end{array}$ & $\begin{array}{l}587 \\
636\end{array}$ & $\begin{array}{l}637 \\
735\end{array}$ \\
\hline 7076820 & $\begin{array}{l}\text { Gum Springs Creek near } \\
\text { H1gginson, Ark. }\end{array}$ & 5.00 & 32.4 & 50 & 305 & 21 & $\begin{array}{l}796 \\
775\end{array}$ & $\begin{array}{l}1,140 \\
1,180\end{array}$ & $\begin{array}{l}1,370 \\
1,550\end{array}$ & $\begin{array}{l}1,650 \\
2,050\end{array}$ & $\begin{array}{l}1,850 \\
2,439\end{array}$ & $\begin{array}{l}2,040 \\
2,920\end{array}$ \\
\hline 7076850 & $\begin{array}{l}\text { Cypress Bayou near Beebe, } \\
\text { Ark. }\end{array}$ & 166 & 2.6 & 50 & 330 & 15 & $\begin{array}{l}6,260 \\
5,760\end{array}$ & $\begin{array}{r}10,900 \\
9,540\end{array}$ & $\begin{array}{l}14,200 \\
12,100\end{array}$ & $\begin{array}{l}18,600 \\
15,700\end{array}$ & $\begin{array}{l}21,800 \\
17,975\end{array}$ & $\begin{array}{l}25,100 \\
21,600\end{array}$ \\
\hline 7076870 & $\begin{array}{l}\text { Pigeon Roost creek at } \\
\text { Butlerville, Ark. }\end{array}$ & 23.0 & 6.9 & 50 & 259 & 24 & $\begin{array}{l}1,980 \\
1,850\end{array}$ & $\begin{array}{l}3,650 \\
3,260\end{array}$ & $\begin{array}{l}5,010 \\
4,340\end{array}$ & $\begin{array}{l}7,010 \\
5,880\end{array}$ & $\begin{array}{l}8,700 \\
7,156\end{array}$ & $\begin{array}{r}10,500 \\
8,750\end{array}$ \\
\hline 7077100 & $\begin{array}{l}\text { Big Creek near Boydsville, } \\
\text { Ark. }\end{array}$ & 12.8 & 20.5 & 47 & 423 & 20 & $\begin{array}{l}2,960 \\
2,700\end{array}$ & $\begin{array}{l}4,300 \\
3,870\end{array}$ & $\begin{array}{l}5,100 \\
4,660\end{array}$ & $\begin{array}{l}6,010 \\
5,690\end{array}$ & $\begin{array}{l}6,630 \\
6,479\end{array}$ & $\begin{array}{l}7,190 \\
7,530\end{array}$ \\
\hline 7077200 & Big Creek tributary near & 1.58 & 40.5 & 47 & 451 & 23 & 376 & 555 & 673 & 820 & 928 & 1,030 \\
\hline 7077340 & $\begin{array}{l}\text { Boydsville, Ark. } \\
\text { Sugar Creek tributary near }\end{array}$ & .68 & 91.3 & 48 & 458 & 22 & $\begin{array}{l}369 \\
286\end{array}$ & $\begin{array}{l}575 \\
468\end{array}$ & $\begin{array}{l}752 \\
594\end{array}$ & $\begin{array}{l}979 \\
753\end{array}$ & $\begin{array}{r}1,166 \\
871\end{array}$ & $\begin{array}{r}1,380 \\
987\end{array}$ \\
\hline & Walcote, Ark. & & & & & & 269 & 438 & 564 & 720 & 853 & 1,000 \\
\hline 7188900 & $\begin{array}{l}\text { Butler Creek tributary near } \\
\text { Gravette, Ark. }\end{array}$ & .96 & 109.0 & 43 & 1,165 & 21 & $\begin{array}{l}104 \\
120\end{array}$ & $\begin{array}{l}297 \\
326\end{array}$ & $\begin{array}{l}482 \\
522\end{array}$ & $\begin{array}{l}772 \\
791\end{array}$ & $\begin{array}{l}1,020 \\
1,022\end{array}$ & $\begin{array}{l}1,290 \\
1,290\end{array}$ \\
\hline 7191220 & $\begin{array}{l}\text { Spavinaw Creek near } \\
\text { Sycamore, Okla. }\end{array}$ & 133 & 20.0 & 43 & 1,204 & 25 & $\begin{array}{l}3,010 \\
3,740\end{array}$ & $\begin{array}{r}8,880 \\
10,400\end{array}$ & $\begin{array}{l}15,300 \\
17,600\end{array}$ & $\begin{array}{l}26,700 \\
28,700\end{array}$ & $\begin{array}{l}38,000 \\
38,168\end{array}$ & $\begin{array}{l}51,800 \\
51,400\end{array}$ \\
\hline 7194890 & $\begin{array}{l}\text { Osage Creek at Cave } \\
\text { Springs, Ark. }\end{array}$ & 40.4 & 19.5 & 43 & 1,300 & 21 & $\begin{array}{l}1,230 \\
1,550\end{array}$ & $\begin{array}{l}3,180 \\
3,950\end{array}$ & $\begin{array}{l}5,090 \\
6,360\end{array}$ & $\begin{array}{l}8,260 \\
9,910\end{array}$ & $\begin{array}{l}11,200 \\
12,710\end{array}$ & $\begin{array}{l}14,600 \\
16,500\end{array}$ \\
\hline 7195000 & $\begin{array}{l}\text { 0sage Creek near Elm } \\
\text { Springs, Ark. }\end{array}$ & 130 & 16.9 & 43 & 1,270 & 30 & $\begin{array}{l}5,010 \\
5,310\end{array}$ & $\begin{array}{l}10,500 \\
11,300\end{array}$ & $\begin{array}{l}15,000 \\
16,500\end{array}$ & $\begin{array}{l}21,400 \\
23,800\end{array}$ & $\begin{array}{l}26,600 \\
29,096\end{array}$ & $\begin{array}{l}32,100 \\
36,300\end{array}$ \\
\hline 7195200 & $\begin{array}{l}\text { Brush Creek tributary near } \\
\text { Tont1town, Ark. }\end{array}$ & .37 & 107.0 & 43 & 1,279 & 21 & 65 & $\begin{array}{l}165 \\
173\end{array}$ & $\begin{array}{l}259 \\
268\end{array}$ & $\begin{array}{l}409 \\
400\end{array}$ & $\begin{array}{l}543 \\
521\end{array}$ & $\begin{array}{l}694 \\
659\end{array}$ \\
\hline 7195450 & $\begin{array}{l}\text { Ballard Creek at Summers, } \\
\text { Ark. }\end{array}$ & 14.6 & 41.0 & 45 & 1,360 & 22 & $\begin{array}{l}1,640 \\
1,680\end{array}$ & $\begin{array}{l}3,800 \\
3,770\end{array}$ & $\begin{array}{l}5,550 \\
5,490\end{array}$ & $\begin{array}{l}7,960 \\
7,750\end{array}$ & $\begin{array}{l}9,840 \\
9,491\end{array}$ & $\begin{array}{l}11,700 \\
11,600\end{array}$ \\
\hline 7195500 & $\begin{array}{l}\text { Illinols River near Watts, } \\
\text { Okla. }\end{array}$ & 635 & 8.5 & 44 & 1,386 & 29 & $\begin{array}{l}17,200 \\
17,600\end{array}$ & $\begin{array}{l}33,000 \\
33,900\end{array}$ & $\begin{array}{l}45,100 \\
47,100\end{array}$ & $\begin{array}{l}61,600 \\
65,400\end{array}$ & $\begin{array}{l}74,600 \\
77,645\end{array}$ & $\begin{array}{l}88,000 \\
95,500\end{array}$ \\
\hline 7195800 & $\begin{array}{l}\text { Flint Creek at Springtown, } \\
\text { Ark. }\end{array}$ & 14.2 & 22.7 & 43 & 1,340 & 24 & $\begin{array}{l}607 \\
738\end{array}$ & $\begin{array}{l}1,880 \\
2,130\end{array}$ & $\begin{array}{l}3,450 \\
3,710\end{array}$ & $\begin{array}{l}6,670 \\
6,420\end{array}$ & $\begin{array}{r}10,300 \\
9,159\end{array}$ & $\begin{array}{l}15,200 \\
13,000\end{array}$ \\
\hline 7196000 & $\begin{array}{l}\text { Flint Creek near Kansas, } \\
\text { okla. }\end{array}$ & 110 & 19.4 & 43 & 1,190 & 27 & $\begin{array}{l}3,400 \\
3,900\end{array}$ & $\begin{array}{r}9,990 \\
10,800\end{array}$ & $\begin{array}{l}17,300 \\
18,100\end{array}$ & $\begin{array}{l}30,800 \\
30,000\end{array}$ & $\begin{array}{l}44,500 \\
41,600\end{array}$ & $\begin{array}{l}61,800 \\
55,600\end{array}$ \\
\hline 7196900 & $\begin{array}{l}\text { Barren Fork at Dutch } \\
\text { M1lls, Ark. }\end{array}$ & 46.0 & 40.2 & 46 & 1,315 & 27 & $\begin{array}{l}6,160 \\
5,990\end{array}$ & $\begin{array}{l}13,000 \\
12,200\end{array}$ & $\begin{array}{l}18,300 \\
17,000\end{array}$ & $\begin{array}{l}25,300 \\
23,200\end{array}$ & $\begin{array}{l}30,600 \\
28,300\end{array}$ & $\begin{array}{l}35,900 \\
33,600\end{array}$ \\
\hline $7247000^{a}$ & $\begin{array}{l}\text { Poceau River at Cauthron, } \\
\text { Ark. }\end{array}$ & 203 & 9.8 & 45 & 830 & 47 & $\begin{array}{l}11,500 \\
11,300\end{array}$ & $\begin{array}{l}19,900 \\
19,500\end{array}$ & $\begin{array}{l}26,100 \\
25,700\end{array}$ & $\begin{array}{l}34,300 \\
34,000\end{array}$ & $\begin{array}{l}40,700 \\
40,800\end{array}$ & $\begin{array}{l}47,100 \\
47,600\end{array}$ \\
\hline 7249300 & $\begin{array}{l}\text { James Fork near Midland, } \\
\text { Ark. }\end{array}$ & 44.0 & 46.5 & 42 & 1,010 & 20 & $\begin{array}{l}5,280 \\
5,060\end{array}$ & $\begin{array}{l}11,100 \\
10,300\end{array}$ & $\begin{array}{l}16,200 \\
14,700\end{array}$ & $\begin{array}{l}24,100 \\
21,100\end{array}$ & $\begin{array}{l}31,000 \\
27,000\end{array}$ & $\begin{array}{l}38,800 \\
33,500\end{array}$ \\
\hline 7249400 & James Fork near Hackett, & 147 & 14.2 & 43 & 770 & 27 & 6,040 & 11,100 & 15,300 & $21,6 \cap 0$ & 27,000 & $33,10 n$ \\
\hline & Ark. & & & & & & 6,260 & 11,800 & 16,900 & 24,200 & 30,500 & 37,200 \\
\hline 7249500 & $\begin{array}{l}\text { Cove Creek near lee Creek, } \\
\text { Ark. }\end{array}$ & 35.3 & 37.0 & 46 & 1,400 & 35 & $\begin{array}{l}4,930 \\
4,830\end{array}$ & $\begin{array}{l}9,450 \\
9,130\end{array}$ & $\begin{array}{l}13,300 \\
12,800\end{array}$ & $\begin{array}{l}19,300 \\
18,200\end{array}$ & $\begin{array}{l}24,600 \\
23,100\end{array}$ & $\begin{array}{l}30,500 \\
28,500\end{array}$ \\
\hline 7249650 & $\begin{array}{l}\text { Mountaln Fork Creek near } \\
\text { Evansville, Ark. }\end{array}$ & 8.15 & 72.8 & 46 & 1,420 & 20 & $\begin{array}{l}1,300 \\
1,290\end{array}$ & $\begin{array}{l}2,470 \\
2,450\end{array}$ & $\begin{array}{l}3,370 \\
3,410\end{array}$ & $\begin{array}{l}4,600 \\
4,670\end{array}$ & $\begin{array}{l}5,570 \\
5,790\end{array}$ & $\begin{array}{l}6,570 \\
6,910\end{array}$ \\
\hline 7250000 & $\begin{array}{l}\text { Cedarville, Ark. } \\
\text { Lee Creek near Van Buren, } \\
\text { Ark. }\end{array}$ & 426 & 17.4 & 45 & 1,070 & 42 & $\begin{array}{r}41 \\
24,300 \\
24,000\end{array}$ & $\begin{array}{l}126 \\
43,200 \\
42,700\end{array}$ & $\begin{array}{l}221 \\
57,700 \\
57,500\end{array}$ & $\begin{array}{r}368 \\
77,700 \\
78,200\end{array}$ & $\begin{array}{r}520 \\
93,700 \\
95,500\end{array}$ & $\begin{array}{r}703 \\
111,000 \\
114,000\end{array}$ \\
\hline 7252000 & $\begin{array}{l}\text { Mulberry River near } \\
\text { Mulberry, Ark. }\end{array}$ & 373 & 18.1 & 48 & 1,430 & 46 & $\begin{array}{l}18,700 \\
18,900\end{array}$ & $\begin{array}{l}34,400 \\
35,000\end{array}$ & $\begin{array}{l}45,600 \\
47,400\end{array}$ & $\begin{array}{l}60,100 \\
64,000\end{array}$ & 70,700 & $\begin{array}{l}81,200 \\
89,800\end{array}$ \\
\hline 7252200 & $\begin{array}{l}\text { North Fork Wh1te Oak Creek } \\
\text { tributary near Watalula, } \\
\text { Ark. }\end{array}$ & .46 & 318.0 & 46 & 920 & 24 & $\begin{array}{l}151 \\
148\end{array}$ & $\begin{array}{l}257 \\
256\end{array}$ & $\begin{array}{l}341 \\
349\end{array}$ & $\begin{array}{l}462 \\
472\end{array}$ & $\begin{array}{r}563 \\
587\end{array}$ & $\begin{array}{l}673 \\
706\end{array}$ \\
\hline 7252500 & $\begin{array}{l}\text { S1xmile Creek subwatershed } \\
\text { No. } 6 \text { near Chismville, Ark. }\end{array}$ & 4.23 & 55.8 & 41 & 720 & 16 & $\begin{array}{l}850 \\
8 \cap 1\end{array}$ & $\begin{array}{l}1,480 \\
1,400\end{array}$ & $\begin{array}{l}1,910 \\
1,870\end{array}$ & $\begin{array}{l}2,470 \\
2,490\end{array}$ & $\begin{array}{l}2,890 \\
3,060\end{array}$ & $\begin{array}{l}3,290 \\
3,610\end{array}$ \\
\hline 7254000 & $\begin{array}{l}\text { Sixmile Creek subwatershed } \\
\text { No. } 5 \text { near Chismville, Ark. }\end{array}$ & 2.76 & 60.3 & 40 & 640 & 18 & $\begin{array}{l}374 \\
381\end{array}$ & $\begin{array}{l}786 \\
802\end{array}$ & $\begin{array}{l}1,110 \\
1,160\end{array}$ & $\begin{array}{l}1,570 \\
1,640\end{array}$ & $\begin{array}{l}1,930 \\
2,070\end{array}$ & $\begin{array}{l}2,300 \\
2,510\end{array}$ \\
\hline 7254500 & Sixmile Creek subwatershed & 5.81 & 19.8 & 41 & 720 & 16 & 870 & 1,450 & 1,860 & 2,400 & 2,820 & 3,240 \\
\hline 7255100 & $\begin{array}{l}\text { No. } 2 \text { near Caulksville, Ark. } \\
\text { Sixmile Creek subwatershed }\end{array}$ & 4.49 & 7.8 & 40 & 460 & 15 & $\begin{array}{l}827 \\
787\end{array}$ & $\begin{array}{l}1,400 \\
1,530\end{array}$ & $\begin{array}{l}1,870 \\
2,080\end{array}$ & $\begin{array}{l}2,490 \\
2,800\end{array}$ & $\begin{array}{l}3,060 \\
3,340\end{array}$ & $\begin{array}{l}3,620 \\
3,880\end{array}$ \\
\hline & 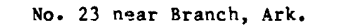 & & & & & & 689 & 1,240 & & 2,060 & 2,460 & 2,890 \\
\hline 7256000 & $\begin{array}{l}\text { Hurricane Creek near } \\
\text { Caulksville, Ark. }\end{array}$ & 53.0 & 23.8 & 40 & 510 & 16 & 3,130 & 5,050 & $\begin{array}{r}6,360 \\
8,220\end{array}$ & 8,040 & $\begin{array}{r}9,280 \\
9\end{array}$ & 10,500 \\
\hline 7256500 & $\begin{array}{l}\text { Spadra Creek at Clarksville, } \\
\text { Ark. }\end{array}$ & 61.1 & 49.3 & 49 & 870 & 34 & $\begin{array}{l}3,260 \\
5,170 \\
5,320\end{array}$ & $\begin{array}{l}5,820 \\
10,400 \\
10,800\end{array}$ & $\begin{array}{r}8,220 \\
14,600 \\
15,400\end{array}$ & $\begin{array}{l}11,500 \\
20,500 \\
21,700\end{array}$ & $\begin{array}{l}14,500 \\
25,400 \\
27,200\end{array}$ & $\begin{array}{l}17,300 \\
30,500 \\
32,700\end{array}$ \\
\hline 7257000 & $\begin{array}{l}\text { Big P1ney Creek near Dover, } \\
\text { Ark. }\end{array}$ & 274 & 17.0 & 49 & 1,410 & 34 & $\begin{array}{l}19,400 \\
19,100\end{array}$ & $\begin{array}{l}37,000 \\
35,900\end{array}$ & $\begin{array}{l}51,500 \\
49,600\end{array}$ & $\begin{array}{l}72,800 \\
69,300\end{array}$ & $\begin{array}{l}90,800 \\
86,300\end{array}$ & $\begin{array}{l}111,000 \\
105,000\end{array}$ \\
\hline
\end{tabular}


Table 2.-Discharge, for selected recurrence 1ntervals, at gaging stations affecting Region B-Cont1nued

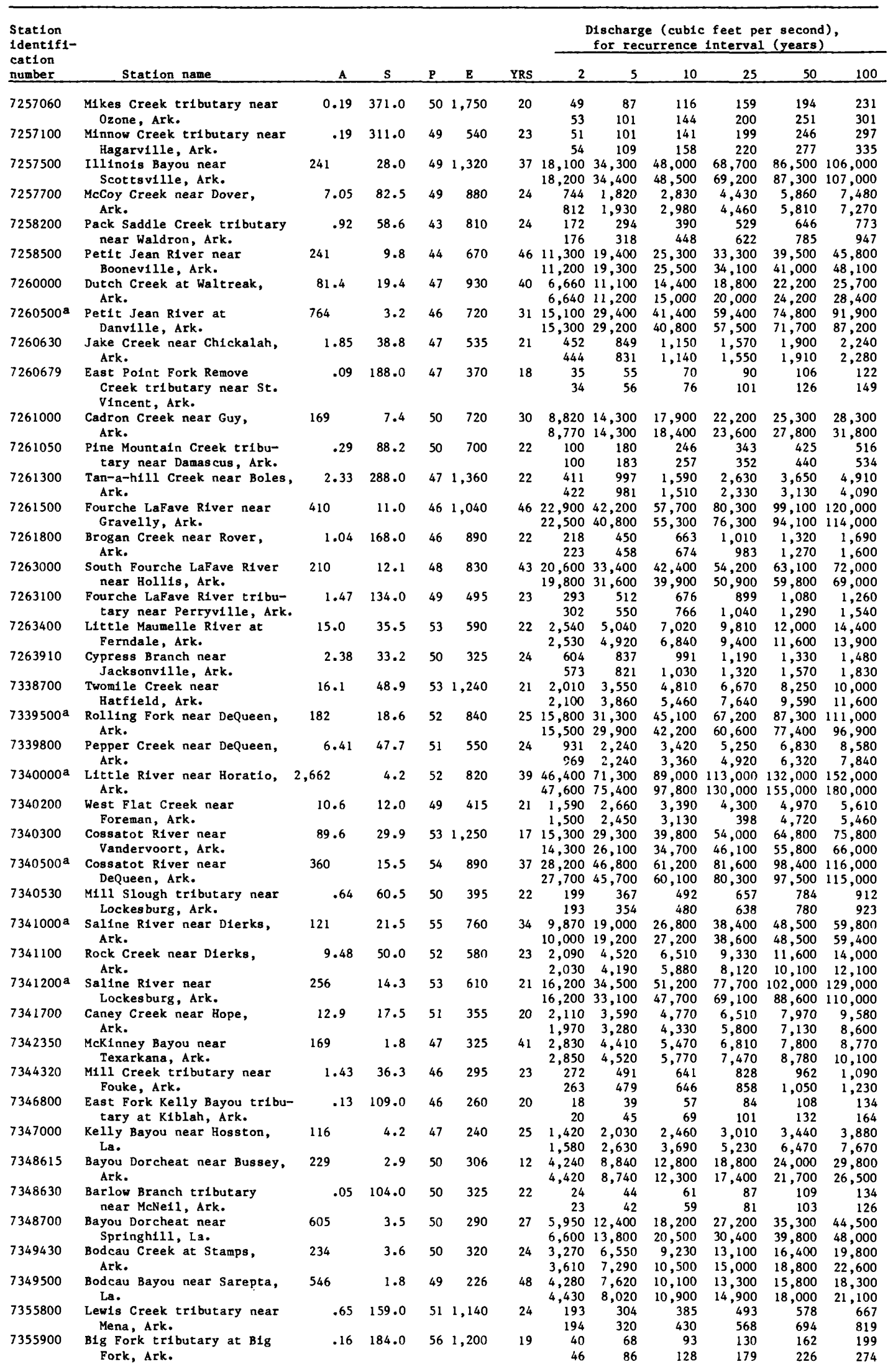


Tabla 2.-Discharge, for selected recurrence intervals, at gaging stations affecting Region B-Continued

\begin{tabular}{|c|c|c|c|c|c|c|c|c|c|c|c|c|}
\hline \multirow{2}{*}{$\begin{array}{l}\text { Station } \\
\text { identifi- } \\
\text { cation } \\
\text { number } \\
\end{array}$} & \multirow[b]{2}{*}{ Station name } & \multirow[b]{2}{*}{ A } & \multirow[b]{2}{*}{$\mathbf{s}$} & \multirow[b]{2}{*}{$P$} & & \multirow[b]{2}{*}{ YRS } & \multicolumn{6}{|c|}{$\begin{array}{l}\text { Discharge (cublc feet per second), } \\
\text { for recurrence interva1 (years) }\end{array}$} \\
\hline & & & & & & & 2 & 2 & 10 & 25 & 0 & 10 \\
\hline 7356000 & Ouachita River near Mount & 414 & 7.8 & 52 & 1,160 & 43 & 22,200 & & & 67,600 & 81,500 & \\
\hline 7356500 & $\begin{array}{l}\text { Ida, Ark. } \\
\text { South Fork Ouachita River } \\
\text { at Mount Ida, Ark. }\end{array}$ & 64.0 & 15.4 & 53 & 830 & 29 & $\begin{array}{r}21,900 \\
6,760 \\
6,640\end{array}$ & $\begin{array}{ll}0 & 37,200 \\
0 & 11,600 \\
0 & 11,300\end{array}$ & $\begin{array}{l}48,900 \\
15,200 \\
15,000\end{array}$ & $\begin{array}{l}65,300 \\
19,900 \\
19,700\end{array}$ & $\begin{array}{l}78,900 \\
23,500 \\
23,600\end{array}$ & $\begin{array}{l}93,500 \\
27,200 \\
27,700\end{array}$ \\
\hline 7356700 & Barnes Branch near Mount & 1.85 & 82.2 & 53 & 760 & 22 & 428 & $8 \quad 862$ & 1,230 & 1,790 & 2,270 & 2,810 \\
\hline $7357501^{a}$ & $\begin{array}{l}\text { Ida, Ark. } \\
\text { Ouachita River at Blakely } \\
\text { Mountain Dam, near Hot } \\
\text { Springs, Ark. }\end{array}$ & 1,100 & 5.2 & 52 & 880 & 16 & $\begin{array}{r}435 \\
42,100 \\
40,200\end{array}$ & $\begin{array}{lr}5 & 867 \\
0 & 67,300 \\
0 & 63,500\end{array}$ & $\begin{array}{r}1,240 \\
85,900 \\
81,400\end{array}$ & $\begin{array}{r}1,740 \\
111,000 \\
107,000\end{array}$ & $\begin{array}{r}2,200 \\
131,000 \\
127,000\end{array}$ & $\begin{array}{l}2,700 \\
152,00 \\
149,001\end{array}$ \\
\hline 7357700 & $\begin{array}{l}\text { Glazypeau Creek at Mountain } \\
\text { valley, Ark. }\end{array}$ & 3.84 & 72.1 & 56 & 810 & 24 & $\begin{array}{l}629 \\
668\end{array}$ & $\begin{array}{ll}9 & 1,230 \\
8 & 1,320\end{array}$ & $\begin{array}{l}1,720 \\
1,900\end{array}$ & $\begin{array}{l}2,420 \\
2,660\end{array}$ & $\begin{array}{l}2,980 \\
3,310\end{array}$ & $\begin{array}{l}3,590 \\
3,980\end{array}$ \\
\hline $7359500^{a}$ & $\begin{array}{l}\text { Ouachita River near } \\
\text { Malvern, Ark. }\end{array}$ & 1,585 & 4.4 & 56 & 810 & 30 & $\begin{array}{l}54,200 \\
53,100\end{array}$ & 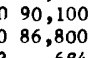 & $\begin{array}{l}115,000 \\
110,000\end{array}$ & $\begin{array}{l}145,000 \\
141,000\end{array}$ & $\begin{array}{l}168,000 \\
165,000\end{array}$ & $\begin{array}{l}190,000 \\
189,000\end{array}$ \\
\hline 7359520 & $\begin{array}{l}\text { Jackson Creek near } \\
\text { Malvern, Ark. }\end{array}$ & 3.00 & 72.1 & 55 & 530 & 20 & $\begin{array}{l}283 \\
357\end{array}$ & $\begin{array}{ll}3 & 684 \\
7 & 853\end{array}$ & $\begin{array}{l}1,080 \\
1,350\end{array}$ & $\begin{array}{l}1,770 \\
2,060\end{array}$ & $\begin{array}{l}2,420 \\
2,720\end{array}$ & $\begin{array}{l}3,210 \\
3,450\end{array}$ \\
\hline 7359700 & $\begin{array}{l}\text { Caddo River at Glenwood, } \\
\text { Ark. }\end{array}$ & 192 & 18.7 & 55 & 970 & 36 & $\begin{array}{l}21,100 \\
20,500\end{array}$ & $\begin{array}{ll}38,000 \\
0 & 36,300\end{array}$ & $\begin{array}{l}50,900 \\
48,100\end{array}$ & $\begin{array}{l}68,600 \\
64,300\end{array}$ & $\begin{array}{l}82,700 \\
77,700\end{array}$ & $\begin{array}{l}97,400 \\
91,900\end{array}$ \\
\hline 7359750 & $\begin{array}{l}\text { Little Sugarloaf Creek } \\
\text { near Bonnerdale, Ark. }\end{array}$ & 2.34 & 86.7 & 58 & 740 & 22 & $\begin{array}{l}807 \\
781\end{array}$ & $\begin{array}{ll}7 & 1,650 \\
1 & 1,530\end{array}$ & $\begin{array}{l}2,340 \\
2,120\end{array}$ & $\begin{array}{l}3,310 \\
2,880\end{array}$ & $\begin{array}{l}4,100 \\
3,550\end{array}$ & $\begin{array}{l}4,940 \\
4,270\end{array}$ \\
\hline 7359800 & $\begin{array}{l}\text { Caddo River near Alplne, } \\
\text { Ark. }\end{array}$ & 312 & 12.3 & 56 & 870 & 28 & $\begin{array}{l}25,900 \\
25,100\end{array}$ & $\begin{array}{ll}0 & 39,500 \\
0 & 38,200\end{array}$ & $\begin{array}{l}48,600 \\
47,900\end{array}$ & $\begin{array}{l}60,000 \\
60,700\end{array}$ & $\begin{array}{l}68,400 \\
71,100\end{array}$ & $\begin{array}{l}76,700 \\
81,500\end{array}$ \\
\hline 7360150 & $\begin{array}{l}\text { Pearson Creek tributary } \\
\text { near Dalark, Ark. }\end{array}$ & .40 & 52.1 & 2 & 340 & 21 & $\begin{array}{l}75 \\
79\end{array}$ & 169 & $\begin{array}{l}266 \\
276\end{array}$ & $\begin{array}{l}440 \\
424\end{array}$ & $\begin{array}{r}615 \\
572\end{array}$ & $\begin{array}{l}837 \\
750\end{array}$ \\
\hline 7360800 & $\begin{array}{l}\text { Muddy Fork Creek near } \\
\text { Murfreesboro, Ark. }\end{array}$ & 121 & 19.2 & 53 & 560 & 41 & $\begin{array}{l}10,900 \\
10,900\end{array}$ & $\begin{array}{ll}0 & 18,700 \\
0 & 18,700\end{array}$ & $\begin{array}{l}24,800 \\
25,100\end{array}$ & $\begin{array}{l}33,400 \\
33,400\end{array}$ & $\begin{array}{l}40,400 \\
41,500\end{array}$ & $\begin{array}{l}48,000 \\
49,400\end{array}$ \\
\hline 7361020 & Prairie Creek tributary & .16 & 217.0 & 54 & 635 & 22 & 69 & $9 \quad 138$ & 198 & 291 & 375 & 471 \\
\hline 7361180 & $\begin{array}{l}\text { near Rirby, Ark. } \\
\text { South Fork Ozan Creek near }\end{array}$ & 17.7 & 16.1 & 52 & 400 & 22 & $\begin{array}{r}69 \\
4,200\end{array}$ & $\begin{array}{r}136 \\
5,860\end{array}$ & $\begin{array}{r}195 \\
6,880\end{array}$ & $\begin{array}{r}273 \\
8,090\end{array}$ & $\begin{array}{r}348 \\
8,940\end{array}$ & $\begin{array}{r}431 \\
9,740\end{array}$ \\
\hline 7361200 & $\begin{array}{l}\text { Ozan, Ark. } \\
\text { Ozan Creek near McCaskill } \\
\text { Ark. }\end{array}$ & 148 & 9.3 & 52 & 420 & 41 & $\begin{array}{l}3,830 \\
7,330 \\
7,360\end{array}$ & $\begin{array}{lr}0 & 5,230 \\
0 & 12,700 \\
0 & 12,800\end{array}$ & $\begin{array}{r}6,170 \\
16,800 \\
17,200\end{array}$ & $\begin{array}{r}7,410 \\
22,500 \\
23,300\end{array}$ & $\begin{array}{r}8,510 \\
27,100 \\
28,300\end{array}$ & $\begin{array}{r}9,630 \\
32,100 \\
33,600\end{array}$ \\
\hline 7361500 & $\begin{array}{l}\text { Antoine River at Antoine, } \\
\text { Ark. }\end{array}$ & 178 & 8.4 & 52 & 520 & 35 & $\begin{array}{l}12,000 \\
11,800\end{array}$ & $\begin{array}{ll}0 & 18,800 \\
0 & 18,400\end{array}$ & $\begin{array}{l}23,600 \\
23,400\end{array}$ & $\begin{array}{l}30,100 \\
30,300\end{array}$ & $\begin{array}{l}35,100 \\
35,900\end{array}$ & $\begin{array}{l}40,300 \\
41,700\end{array}$ \\
\hline 7361680 & $\begin{array}{l}\text { Middle Caney Creek tribu- } \\
\text { tary near Rosston, Ark. }\end{array}$ & 1.48 & 47.9 & 51 & 357 & 24 & $\begin{array}{l}225 \\
237\end{array}$ & $\begin{array}{l}5 \\
7\end{array} 463$ & $\begin{array}{l}663 \\
714\end{array}$ & $\begin{array}{r}958 \\
1,020\end{array}$ & $\begin{array}{l}1,210 \\
1,300\end{array}$ & $\begin{array}{l}1,480 \\
1,590\end{array}$ \\
\hline 7361780 & $\begin{array}{l}\text { Bradshaw Creek near } \\
\text { Hollywood, Ark. }\end{array}$ & 3.46 & 21.9 & 52 & 320 & 20 & $\begin{array}{l}473 \\
473\end{array}$ & $\begin{array}{ll}3 & 730 \\
3 & 777\end{array}$ & $\begin{array}{r}917 \\
1,050\end{array}$ & $\begin{array}{l}1,170 \\
1,420\end{array}$ & $\begin{array}{l}1,370 \\
1,750\end{array}$ & $\begin{array}{l}1,580 \\
2,080\end{array}$ \\
\hline 7361800 & $\begin{array}{l}\text { Terre No1re Creek east of } \\
\text { Gurdon, Ark. }\end{array}$ & 250 & 7.4 & 52 & 296 & 41 & $\begin{array}{l}16,900 \\
16,100\end{array}$ & $\begin{array}{ll}0 & 24,000 \\
0 & 22,600\end{array}$ & $\begin{array}{l}28,800 \\
27,200\end{array}$ & $\begin{array}{l}34,900 \\
33,400\end{array}$ & $\begin{array}{l}39,500 \\
38,500\end{array}$ & $\begin{array}{l}44,000 \\
43,700\end{array}$ \\
\hline 7362050 & $\begin{array}{l}\text { Ross Creek near Camden, } \\
\text { Ark. }\end{array}$ & 10.4 & 17.5 & 50 & 202 & 21 & $\begin{array}{r}386 \\
434\end{array}$ & $\begin{array}{rr}6 & 903 \\
4 & 1,020\end{array}$ & $\begin{array}{l}1,380 \\
1,600\end{array}$ & $\begin{array}{l}2,140 \\
2,430\end{array}$ & $\begin{array}{l}2,810 \\
3,180\end{array}$ & $\begin{array}{l}3,580 \\
3,990\end{array}$ \\
\hline 7362100 & $\begin{array}{l}\text { Smackover Creek near } \\
\text { Smackover, Ark. }\end{array}$ & 385 & 4.0 & 50 & 230 & 46 & $\begin{array}{l}5,860 \\
5,940\end{array}$ & $\begin{array}{ll}0 & 11,800 \\
0 & 11,900\end{array}$ & $\begin{array}{l}17,000 \\
17,100\end{array}$ & $\begin{array}{l}24,900 \\
24,800\end{array}$ & $\begin{array}{l}31,800 \\
31,500\end{array}$ & $\begin{array}{l}39,600 \\
39,000\end{array}$ \\
\hline 7362330 & $\begin{array}{l}\text { Dunn Creek near Hampton, } \\
\text { Ark. }\end{array}$ & 13.6 & 11.2 & 50 & 187 & 23 & $\begin{array}{l}743 \\
731\end{array}$ & $\begin{array}{ll}3 & 1,740 \\
1 & 1,640\end{array}$ & $\begin{array}{l}2,680 \\
2,450\end{array}$ & $\begin{array}{l}4,210 \\
3,670\end{array}$ & $\begin{array}{l}5,620 \\
4,800\end{array}$ & $\begin{array}{l}7,260 \\
6,130\end{array}$ \\
\hline 7362450 & $\begin{array}{l}\text { Cooks Creek near Fordyce, } \\
\text { Ark. }\end{array}$ & 4.99 & 20.0 & 51 & 315 & 20 & $\begin{array}{l}665 \\
652\end{array}$ & $\begin{array}{l}1,290 \\
1,250\end{array}$ & $\begin{array}{l}1,780 \\
1,730\end{array}$ & $\begin{array}{r}2,440 \\
2,360\end{array}$ & $\begin{array}{l}2,960 \\
2,910\end{array}$ & $\begin{array}{l}3,490 \\
3,480\end{array}$ \\
\hline 7362500 & $\begin{array}{l}\text { Moro Creek near Fordyce, } \\
\text { Ark. }\end{array}$ & 240 & 5.6 & 51 & 270 & 34 & $\begin{array}{r}4,520 \\
4,710\end{array}$ & $\begin{array}{ll}0 & 9,090 \\
0 & 9,480\end{array}$ & $\begin{array}{l}12,700 \\
13,400\end{array}$ & $\begin{array}{l}17,700 \\
19,000\end{array}$ & $\begin{array}{l}21,700 \\
23,500\end{array}$ & $\begin{array}{l}25,800 \\
28,100\end{array}$ \\
\hline 7363000 & $\begin{array}{l}\text { Saline Rivar at Benton, } \\
\text { Ark. }\end{array}$ & 550 & 12.4 & 54 & 650 & 47 & $\begin{array}{l}29,600 \\
29,500\end{array}$ & $\begin{array}{ll}0 & 49,400 \\
0 & 49,500\end{array}$ & $\begin{array}{l}63,500 \\
64,500\end{array}$ & $\begin{array}{l}81,700 \\
84,600\end{array}$ & $\begin{array}{r}95,400 \\
100,000\end{array}$ & $\begin{array}{l}109,000 \\
116,000\end{array}$ \\
\hline 7363050 & $\begin{array}{l}\text { Holly Creek tributary near } \\
\text { Benton, Ark. }\end{array}$ & 1.44 & 47.0 & 53 & 345 & 23 & $\begin{array}{l}184 \\
201\end{array}$ & $\begin{array}{l}4 \\
1 \quad 406 \\
\end{array}$ & $\begin{array}{l}634 \\
694\end{array}$ & $\begin{array}{l}1,040 \\
1,070\end{array}$ & $\begin{array}{l}1,460 \\
1,450\end{array}$ & $\begin{array}{l}1,980 \\
1,890\end{array}$ \\
\hline 7363200 & $\begin{array}{l}\text { Saline River near Sheridan, } \\
\text { Ark. }\end{array}$ & 1,129 & 4.5 & 54 & 460 & 47 & $\begin{array}{l}25,300 \\
25,700\end{array}$ & $\begin{array}{ll}0 & 40,800 \\
0 & 42,100\end{array}$ & & $\begin{array}{l}65,600 \\
71,700\end{array}$ & $\begin{array}{l}76,000 \\
84,600\end{array}$ & $\begin{array}{l}86,500 \\
97,500\end{array}$ \\
\hline 7363300 & $\begin{array}{l}\text { Hurricane Creek near } \\
\text { Sheridan, Ark. }\end{array}$ & 204 & 6.9 & 53 & 375 & 24 & $\begin{array}{l}7,760 \\
7,820\end{array}$ & $\begin{array}{ll}0 & 15,700 \\
0 & 15,400\end{array}$ & $\begin{array}{l}21,900 \\
21,200\end{array}$ & $\begin{array}{l}30,500 \\
29,200\end{array}$ & $\begin{array}{l}37,200 \\
35,700\end{array}$ & $\begin{array}{l}44,000 \\
42,400\end{array}$ \\
\hline 7363330 & $\begin{array}{l}\text { West Fork 81g Creek at } \\
\text { Sheridan, Ark. }\end{array}$ & 4.86 & 22.2 & 53 & 290 & 22 & $\begin{array}{l}446 \\
469\end{array}$ & $\begin{array}{r}6 \\
9 \\
9 \\
1,000\end{array}$ & $\begin{array}{l}1,390 \\
1,480\end{array}$ & $\begin{array}{l}2,040 \\
2,130\end{array}$ & $\begin{array}{l}2,580 \\
2,710\end{array}$ & $\begin{array}{l}3,160 \\
3,310\end{array}$ \\
\hline 7363430 & $\begin{array}{l}\text { East Fork Derrieusseaux } \\
\text { Creek near Pine Bluff, } \\
\text { Ark. }\end{array}$ & .64 & 64.5 & 51 & 350 & 21 & $\begin{array}{l}118 \\
123\end{array}$ & $\begin{array}{l}260 \\
269\end{array}$ & $\begin{array}{l}393 \\
405\end{array}$ & $\begin{array}{l}609 \\
597\end{array}$ & $\begin{array}{l}807 \\
779\end{array}$ & $\begin{array}{r}1,040 \\
984\end{array}$ \\
\hline 7363450 & $\begin{array}{l}\text { Varnell Creek near Rison, } \\
\text { Ark. }\end{array}$ & .28 & 63.3 & 51 & 243 & 21 & $\begin{array}{r}47 \\
49\end{array}$ & $\begin{array}{ll}7 & 111 \\
9 & 114\end{array}$ & $\begin{array}{l}168 \\
172\end{array}$ & $\begin{array}{l}252 \\
250\end{array}$ & $\begin{array}{l}323 \\
321\end{array}$ & $\begin{array}{l}400 \\
398\end{array}$ \\
\hline 7363500 & $\begin{array}{l}\text { Saline River near Rye, } \\
\text { Ark. }\end{array}$ & 2,102 & 2.4 & 52 & 360 & 47 & $\begin{array}{l}23,700 \\
24,300\end{array}$ & $\begin{array}{ll}0 & 41,800 \\
0 & 43,000\end{array}$ & $\begin{array}{l}55,000 \\
57,400\end{array}$ & $\begin{array}{l}72,700 \\
77,400\end{array}$ & $\begin{array}{l}86,300 \\
92,700\end{array}$ & $\begin{array}{l}100,000 \\
108,000\end{array}$ \\
\hline 7364030 & $\begin{array}{l}\text { L'Algle Creek tributary near } \\
\text { Hermitage, Ark. }\end{array}$ & .36 & 48.3 & 52 & 170 & 22 & $\begin{array}{l}36 \\
39\end{array}$ & $\begin{array}{ll}6 & 77 \\
9 & 87\end{array}$ & $\begin{array}{l}109 \\
132\end{array}$ & $\begin{array}{l}151 \\
189\end{array}$ & $\begin{array}{l}183 \\
240\end{array}$ & $\begin{array}{l}214 \\
291\end{array}$ \\
\hline 7364070 & $\begin{array}{l}\text { Bear Creek near Strong, } \\
\text { Ark. }\end{array}$ & 5.62 & 15.2 & 32 & 165 & 21 & $\begin{array}{l}337 \\
341\end{array}$ & $\begin{array}{l}541 \\
589\end{array}$ & $\begin{array}{l}680 \\
810\end{array}$ & $\begin{array}{r}855 \\
1,110\end{array}$ & $\begin{array}{r}985 \\
1,380\end{array}$ & $\begin{array}{l}1,110 \\
1,640\end{array}$ \\
\hline 364260 & $\begin{array}{l}\text { Hanks Creek near Hamburg, } \\
\text { Ark. }\end{array}$ & 20.9 & 6.5 & 54 & 170 & 22 & $\begin{array}{l}686 \\
697\end{array}$ & $\begin{array}{l}1,270 \\
7,310\end{array}$ & $\begin{array}{l}1,700 \\
1,810\end{array}$ & $\begin{array}{l}2,260 \\
2,490\end{array}$ & & $\begin{array}{l}3,120 \\
3,630\end{array}$ \\
\hline 7364300 & $\begin{array}{l}\text { Chemin-a-Haut Bayou near } \\
\text { Beekman, La. }\end{array}$ & 271 & 3.3 & $\pi$ & 142 & 24 & $\begin{array}{l}4,860 \\
4,690\end{array}$ & $\begin{array}{r}10,400 \\
0,590\end{array}$ & $\begin{array}{l}15,000 \\
13,400\end{array}$ & $\begin{array}{l}21,800 \\
19,000\end{array}$ & $\begin{array}{l}27,500 \\
23,700\end{array}$ & $\begin{array}{l}33,500 \\
28,900\end{array}$ \\
\hline 7364550 & $\begin{array}{l}\text { Caney Creek tributary near } \\
\text { E1 Dorado, Ark. }\end{array}$ & .13 & 222.0 & $\rightarrow 7$ & 235 & 23 & $\begin{array}{l}72 \\
65\end{array}$ & $\begin{array}{l}132 \\
115\end{array}$ & $\begin{array}{l}186 \\
158\end{array}$ & $\begin{array}{l}274 \\
221\end{array}$ & $\begin{array}{l}354 \\
283\end{array}$ & $\begin{array}{l}450 \\
357\end{array}$ \\
\hline 7364700 & $\begin{array}{l}\text { Bayou de Loutre near } \\
\text { Laran, La. }\end{array}$ & 141 & 3.6 & 49 & 203 & 22 & $\begin{array}{l}2,490 \\
2,510\end{array}$ & $\begin{array}{l}5,580 \\
5,410\end{array}$ & $\begin{array}{l}8,770 \\
8,180\end{array}$ & $\begin{array}{l}14,500 \\
12,700\end{array}$ & $\begin{array}{l}20,400 \\
17,200\end{array}$ & $\begin{array}{l}28,000 \\
22,800\end{array}$ \\
\hline 7365800 & $\begin{array}{l}\text { Cornile Bayou near Three } \\
\text { Creeks, Ark. }\end{array}$ & 180 & 5.1 & 48 & 250 & 29 & $\begin{array}{l}4,030 \\
4,050\end{array}$ & $\begin{array}{l}9,790 \\
9,390\end{array}$ & $\begin{array}{l}15,700 \\
14,500\end{array}$ & $\begin{array}{l}26,300 \\
22,900\end{array}$ & & $\begin{array}{l}50,200 \\
41,500\end{array}$ \\
\hline 7365900 & $\begin{array}{l}\text { Three Creeks near Three } \\
\text { Creeks, Ark. }\end{array}$ & 50.3 & 6.2 & 46 & 245 & 23 & $\begin{array}{l}2,110 \\
2,040\end{array}$ & $\begin{array}{l}4,790 \\
4,400\end{array}$ & $\begin{array}{l}7,430 \\
6,520\end{array}$ & $\begin{array}{r}11,900 \\
9,840\end{array}$ & & $\begin{array}{l}21,600 \\
17,000\end{array}$ \\
\hline 7366000 & $\begin{array}{l}\text { Corney Bayou near Lillle, } \\
\text { La. }\end{array}$ & 462 & 3.5 & 48 & 185 & 43 & $\begin{array}{l}5,930 \\
5,930\end{array}$ & $\begin{array}{ll}0 & 11,500 \\
0 & 11,500\end{array}$ & $\begin{array}{l}16,500 \\
16,400\end{array}$ & $\begin{array}{l}24,300 \\
23,900\end{array}$ & $\begin{array}{l}31,500 \\
30,800\end{array}$ & $\begin{array}{l}39,700 \\
38,500\end{array}$ \\
\hline 7366200 & $\begin{array}{l}\text { Little Corney Bayou near } \\
\text { Lillie, La. }\end{array}$ & 208 & 3.7 & 48 & 201 & 29 & $\begin{array}{l}3,210 \\
3,240 \\
\end{array}$ & $\begin{array}{r}0,870 \\
0 \quad 6,790 \\
\end{array}$ & $\begin{array}{r}10,100 \\
9,870 \\
\end{array}$ & $\begin{array}{l}15,200 \\
14,500 \\
\end{array}$ & $\begin{array}{l}19,700 \\
18,600 \\
\end{array}$ & $\begin{array}{l}24,800 \\
23,200 \\
\end{array}$ \\
\hline
\end{tabular}

a Before regulation; regulated values are shown in table 3. 
Table 3.-D1scharge, for selected recurrence 1ntervals, on large streams and regulated streams

[A, drainage area; YRS, years of record. Numbers on line with station name are based on station data.]

\begin{tabular}{|c|c|c|c|c|c|c|c|c|c|}
\hline \multirow{2}{*}{$\begin{array}{l}\text { Station } \\
\text { Ident1f1- } \\
\text { cation } \\
\text { number } \\
\end{array}$} & \multirow[b]{2}{*}{ Station name } & \multirow[b]{2}{*}{$\mathbf{A}$} & \multirow[b]{2}{*}{ YRS } & \multicolumn{6}{|c|}{$\begin{array}{l}\text { Discharge (cubic feet per second), } \\
\text { for recurrence interval (years) }\end{array}$} \\
\hline & & & & 2 & 5 & 10 & 25 & 50 & 100 \\
\hline $7032000^{a}$ & $\begin{array}{l}\text { Mississippl River at } \\
\text { Memphis, Tenn. }\end{array}$ & 932,800 & 52 & 1,170 & 1,410 & 1,540 & 1,680 & 1,770 & 1,840 \\
\hline 7040100 & $\begin{array}{l}\text { St. Francis River at } \\
\text { St. Francls, Ark. }\end{array}$ & 1,772 & 39 & 10,600 & 15,300 & 18,600 & 22,800 & 26,000 & 29,300 \\
\hline 7040450 & $\begin{array}{l}\text { St. Francis River at } \\
\text { Lake City, Ark. }\end{array}$ & 2,374 & 39 & 14,100 & 20,500 & 24,900 & 30,700 & 35,100 & 39,700 \\
\hline 7046600 & $\begin{array}{l}\text { R1ght hand chute of Little } \\
\text { River at Rivervale, Ark. }\end{array}$ & 2,106 & 36 & 14,900 & 24,500 & 30,900 & 38,800 & 44,600 & 50,100 \\
\hline 7047000 & $\begin{array}{l}\text { St. Francis River floodway } \\
\text { near Marked Tree, Ark. }\end{array}$ & 0 & 36 & 19,200 & 32,000 & 40,500 & 51,000 & 58,500 & 65,700 \\
\hline 7047500 & $\begin{array}{l}\text { St. Francis River at } \\
\text { Marked Tree, Ark. }\end{array}$ & 0 & 39 & 3,530 & 4,320 & 4,820 & 5,420 & 5,850 & 6,270 \\
\hline $7047501^{b}$ & $\begin{array}{l}\text { St. Francis River and St. } \\
\text { Francis River floodway } \\
\text { near Marked Tree, Ark. }\end{array}$ & 5,148 & 36 & 22,000 & 35,400 & 44,300 & 55,100 & 62,800 & 70,200 \\
\hline 7047950 & $\begin{array}{l}\text { L'Anguille River at } \\
\text { Palestine, Ark. }\end{array}$ & 786 & 32 & 8,570 & 12,700 & 15,100 & 18,000 & 20,000 & 21,800 \\
\hline 7047800 & $\begin{array}{l}\text { St. Franc1s River at } \\
\text { Parkin, Ark. }\end{array}$ & 0 & 49 & 10,000 & 13,500 & 15,700 & 18,400 & 20,400 & 22,200 \\
\hline 7047900 & $\begin{array}{l}\text { St. Francis Bay at } \\
\text { Riverfront, Ark. }\end{array}$ & 0 & 46 & 21,100 & 33,000 & 40,900 & 50,500 & 57,400 & 64,100 \\
\hline $7047902^{c}$ & $\begin{array}{l}\text { St. Francis River at Parkin } \\
\text { and St. Francies Bay at } \\
\text { Riverfront combined }\end{array}$ & 6,475 & 42 & 26,700 & 39,500 & 47,700 & 57,600 & 64,700 & 71,500 \\
\hline $7047970^{a}$ & $\begin{array}{l}\text { M1ssiss1pp1 R1ver at } \\
\text { Helena, Ark. }\end{array}$ & 941,700 & 54 & 1,200 & 1,450 & 1,580 & 1,730 & 1,830 & 1,920 \\
\hline 7049691 & $\begin{array}{l}\text { White River at Beaver Dam } \\
\text { near Eureka Springs, Ark. }\end{array}$ & 1,192 & 19 & 6,320 & 9,720 & 12,400 & 16,100 & 19,200 & 22,600 \\
\hline 7055000 & $\begin{array}{l}\text { White River near Filppin, } \\
\text { Ark. }\end{array}$ & 6,067 & 33 & 25,200 & 29,400 & 31,300 & 33,100 & 34,200 & 35,000 \\
\hline 7060000 & $\begin{array}{l}\text { North Fork River at Norfork } \\
\text { Dam, near Norfork, Ark. }\end{array}$ & 1,806 & 40 & 5,540 & 8,310 & 10,300 & 13,100 & 15,400 & 17,700 \\
\hline 7060500 & $\begin{array}{l}\text { White River at Calico Rock, } \\
\text { Ark. }\end{array}$ & 9,978 & 42 & 62,900 & 115,000 & 158,000 & 224,000 & 282,000 & 348,000 \\
\hline 7061000 & $\begin{array}{l}\text { White River at Batesville, } \\
\text { Ark. }\end{array}$ & 11,062 & 14 & 64,900 & 114,000 & 153,000 & 211,000 & 260,000 & 314,000 \\
\hline 7064000 & $\begin{array}{l}\text { Black River near Corning, } \\
\text { Ark. }\end{array}$ & 1,749 & 36 & 11,800 & 20,000 & 25,900 & 33,700 & 39,600 & 45,500 \\
\hline 7069000 & $\begin{array}{l}\text { Black River at Pocahontas, } \\
\text { Ark. }\end{array}$ & 4,845 & 48 & 23,400 & 39,200 & 51,500 & 69,400 & 84,200 & 100,000 \\
\hline 7072500 & $\begin{array}{l}\text { Black River at Black Rock, } \\
\text { Ark. }\end{array}$ & 7,369 & 36 & 44,700 & 76,200 & 101,000 & 136,000 & 164,000 & 195,000 \\
\hline $\begin{array}{l}7074500 \\
7076000\end{array}$ & $\begin{array}{l}\text { White River at Newport, Ark. } \\
\text { Little Red River near } \\
\text { Heber Springs, Ark. }\end{array}$ & $\begin{array}{r}19,860 \\
1,146\end{array}$ & $\begin{array}{l}41 \\
23\end{array}$ & $\begin{array}{r}82,900 \\
8,400\end{array}$ & $\begin{array}{r}143,000 \\
8,960\end{array}$ & $\begin{array}{r}191,000 \\
9,290\end{array}$ & $\begin{array}{r}262,000 \\
9,670\end{array}$ & $\begin{array}{r}323,000 \\
9,930\end{array}$ & $\begin{array}{r}389,000 \\
10,200\end{array}$ \\
\hline 7077000 & $\begin{array}{l}\text { White River at DeValls } \\
\text { Bluff, Ark. }\end{array}$ & 23,483 & 41 & 875,00 & 125,000 & 149,000 & 177,000 & 198,000 & 217,000 \\
\hline 7077800 & $\begin{array}{l}\text { White River at Clarendon, } \\
\text { Ark. }\end{array}$ & 25,555 & 28 & 81,600 & 118,000 & 143,000 & 174,000 & 197,000 & 221,000 \\
\hline 7247000 & $\begin{array}{l}\text { Poteau River at Cauthron } \\
\text { Ark. }\end{array}$ & 203 & 12 & 8,510 & 12,000 & 14,400 & 17,400 & 19,600 & 21,800 \\
\hline 7250550 & $\begin{array}{l}\text { Arkansas River at Dam No. } \\
13 \text { near Van Buren, Ark. }\end{array}$ & 150,547 & 15 & 144,000 & 189,000 & 218,000 & 251,000 & 275,000 & 298,000 \\
\hline $7250550^{d}$ & $\begin{array}{l}\text { Arkansas River at Dan No. } \\
13 \text { near Van Buren, Ark. }\end{array}$ & 150,547 & 45 & 155,000 & 218,000 & 264,000 & 324,000 & 372,000 & 420,000 \\
\hline 7251000 & $\begin{array}{l}\text { Frog Bayou near } \\
\text { Mountalnburg, Ark. }\end{array}$ & 74.2 & 25 & 4,530 & 8,620 & 11,500 & 15,200 & 17,800 & 20,400 \\
\hline $\begin{array}{l}7251500 \\
7253000\end{array}$ & $\begin{array}{l}\text { Frog Bayou at Rudy, Ark. } \\
\text { Sixmile Creek at } \\
\text { Chismille, Ark. }\end{array}$ & 216 & $\begin{array}{l}36 \\
16\end{array}$ & $\begin{array}{r}11,100 \\
1,090\end{array}$ & $\begin{array}{r}20,400 \\
2,130\end{array}$ & $\begin{array}{r}26,900 \\
2,900\end{array}$ & $\begin{array}{r}35,200 \\
3,910\end{array}$ & $\begin{array}{r}41,300 \\
4,680\end{array}$ & $\begin{array}{r}47,200 \\
5,440\end{array}$ \\
\hline 7253500 & $\begin{array}{l}\text { S1xmile Creek near } \\
\text { Branch, Ark. }\end{array}$ & 36.7 & 16 & 2,150 & 3,800 & 4,980 & 6,520 & 7,660 & 8,810 \\
\hline 7255000 & $\begin{array}{l}\text { S1xmile Creek at } \\
\text { Caulksville, Ark. }\end{array}$ & 104 & 16 & 4,940 & 7,830 & 9,810 & 12,300 & 14,200 & 16,100 \\
\hline 7255500 & $\begin{array}{l}\text { Hurricane Creek near } \\
\text { Branch, Ark. }\end{array}$ & 17.2 & 16 & 748 & 1,440 & 1,970 & 2,700 & 3,270 & 3,850 \\
\hline 7258000 & $\begin{array}{c}\text { Arkansas River at } \\
\text { Dardane } 11 \mathrm{e} \text {, Ark. }\end{array}$ & 153,670 & 45 & 201,000 & 320,000 & 405,000 & 518,000 & 605,000 & 696,000 \\
\hline 7259500 & $\begin{array}{l}\text { Petit Jean River near } \\
\text { Waveland, Ark. }\end{array}$ & 516 & 37 & 3,500 & 5,260 & 6,510 & 8,200 & 9,520 & 10,900 \\
\hline 7260500 & $\begin{array}{l}\text { Petit Jean River at } \\
\text { Danville, Ark. }\end{array}$ & 764 & 37 & 8,650 & 15,900 & 21,900 & 30,700 & 38,300 & 46,700 \\
\hline 7262500 & $\begin{array}{l}\text { Fourche LaFave River near } \\
\text { Nimrod, Ark. }\end{array}$ & 684 & 42 & 5,750 & 7,450 & 8,660 & 10,300 & 11,600 & 12,900 \\
\hline 7263450 & $\begin{array}{l}\text { Arkansas River at Murray } \\
\text { Dam at Little Rock, Ark. }\end{array}$ & 158,030 & 45 & 203,000 & 306,000 & 373,000 & 458,000 & 521,000 & 582,000 \\
\hline 7264500 & $\begin{array}{l}\text { Bayou Meto near Stuttgart, } \\
\text { Ark. }\end{array}$ & 574 & 45 & 1,930 & 2,790 & 3,410 & 4,250 & 4,900 & 5,600 \\
\hline 7265000 & $\begin{array}{l}\text { Crooked Creek near } \\
\text { Humphrey, Ark. }\end{array}$ & 79.2 & 40 & 1,380 & 1,930 & 2,230 & 2,560 & 2,780 & 2,960 \\
\hline
\end{tabular}


Table 3.-D1scharge, for selected recurrence intervals, on large streams and regulated streams-Continued

\begin{tabular}{|c|c|c|c|c|c|c|c|c|c|}
\hline \multirow{2}{*}{$\begin{array}{l}\text { Station } \\
\text { identifi- } \\
\text { cation } \\
\text { number }\end{array}$} & \multirow[b]{2}{*}{ Station name } & \multirow[b]{2}{*}{$\mathbf{A}$} & \multirow[b]{2}{*}{ YRS } & \multicolumn{6}{|c|}{$\begin{array}{l}\text { nischarge (cubic feet per second), } \\
\text { for recurrence interval (years) }\end{array}$} \\
\hline & & & & 2 & 5 & 10 & 25 & 50 & 100 \\
\hline 7265001 & $\begin{array}{l}\text { Bayou Meto and Crooked } \\
\text { Creek near Stuttgart, } \\
\text { Ark. }\end{array}$ & 653 & 45 & 3,330 & 4,750 & 5,640 & 6,700 & 7,460 & 8,190 \\
\hline $7265450^{a}$ & $\begin{array}{l}\text { Mississippi River near } \\
\text { Arkansas City, Ark. }\end{array}$ & 130,600 & 54 & 1,320 & 1,620 & 1,800 & 2,010 & 2,150 & 2,290 \\
\hline 7337000 & Red River at Index, Ark. & 48,030 & 42 & 76,000 & 109,000 & 130,000 & 156,000 & 174,000 & 192,000 \\
\hline 7339500 & $\begin{array}{l}\text { Rolling Fork near DeQueen, } \\
\text { Ark. }\end{array}$ & 182 & 11 & 3,460 & 5,440 & 6,830 & 8,640 & 10,000 & 11,400 \\
\hline 7340000 & $\begin{array}{l}\text { Little River near Horatio, } \\
\text { Ark. }\end{array}$ & 2,674 & 16 & 27,700 & 41,900 & 51,500 & 63,700 & 72,800 & 81,800 \\
\hline 7340500 & $\begin{array}{l}\text { Cossatot River near } \\
\text { DeQueen, Ark. }\end{array}$ & 361 & 10 & 8,020 & 14,000 & 18,600 & 24,800 & 29,800 & 35,000 \\
\hline 7341000 & $\begin{array}{l}\text { Saline River near Dierks } \\
\text { Ark. }\end{array}$ & 124 & 12 & 2,690 & 5,450 & 7,880 & 11,700 & 15,100 & 19,000 \\
\hline 7341200 & $\begin{array}{l}\text { Saline River near } \\
\text { Lockesburg, Ark. }\end{array}$ & 260 & 11 & 10,000 & 19,300 & 27,400 & 40,200 & 51,800 & 65,200 \\
\hline 7341301 & $\begin{array}{l}\text { Little River at Millwood } \\
\text { Dam near Ashdown, Ark. }\end{array}$ & 4,119 & 18 & 33,500 & 50,100 & 61,300 & 75,600 & 86,200 & 96,900 \\
\hline $\begin{array}{l}7341500 \\
7348500\end{array}$ & $\begin{array}{l}\text { Red River at Fulton, Ark. } \\
\text { Red River at Shreveport, }\end{array}$ & $\begin{array}{l}52,380 \\
60,613\end{array}$ & $\begin{array}{l}31 \\
37\end{array}$ & $\begin{array}{r}90,100 \\
114,000\end{array}$ & $\begin{array}{l}131,000 \\
164,000\end{array}$ & $\begin{array}{l}159,000 \\
196,000\end{array}$ & $\begin{array}{l}195,000 \\
237,000\end{array}$ & $\begin{array}{l}223,000 \\
267,000\end{array}$ & $\begin{array}{l}251,000 \\
297,000\end{array}$ \\
\hline 7357501 & $\begin{array}{l}\text { Ouachita River at Blakely } \\
\text { Mountain Dam near Hot } \\
\text { Springs, Ark. }\end{array}$ & 1,105 & 31 & 6,590 & 8,610 & 9,730 & 11,000 & 11,800 & 12,500 \\
\hline 7359500 & $\begin{array}{l}\text { Ouachica River near } \\
\text { Malvern, Ark. }\end{array}$ & 1,585 & 32 & 28,200 & 51,200 & 71,300 & 103,000 & 131,000 & 164,000 \\
\hline 7359910 & $\begin{array}{l}\text { Caddo River at DeGray regu- } \\
\text { lating dam near } \\
\text { Arkadelphia, Ark. }\end{array}$ & & & & & & & & \\
\hline 7360000 & $\begin{array}{l}\text { Ouachita River at } \\
\text { Arkadelphia, Ark. }\end{array}$ & 2,311 & 33 & 39,300 & 68,000 & 90,000 & 121,000 & 146,000 & 172,000 \\
\hline 7360501 & $\begin{array}{l}\text { Little Missouri River at } \\
\text { Narrows Dam near } \\
\text { Murfressboro, Ark. }\end{array}$ & 237 & 34 & 2,600 & 3,740 & 4,530 & 5,560 & 6,360 & 7,170 \\
\hline 7361000 & $\begin{array}{l}\text { Little Missouri River near } \\
\text { Murfreesboro, Ark. }\end{array}$ & 380 & 28 & 12,600 & 20,000 & 25,100 & 31,500 & 36,200 & 40,800 \\
\hline 7361600 & $\begin{array}{l}\text { Little Missouri River near } \\
\text { Boughton, Ark. }\end{array}$ & 1,068 & 32 & 26,800 & 41,800 & 51,600 & 63,600 & 72,100 & 80,400 \\
\hline 7362000 & $\begin{array}{l}\text { Ouachita River at Camden, } \\
\text { Ark. }\end{array}$ & 5,357 & 32 & 58,300 & 101,000 & 134,000 & 178,000 & 213,000 & 250,000 \\
\hline 7364000 & $\begin{array}{l}\text { Saline River near Warren, } \\
\text { Ark. }\end{array}$ & 2,476 & 13 & 19,400 & 34,800 & 46,600 & 62,900 & 75,900 & 89,600 \\
\hline 7367661 & $\begin{array}{l}\text { Boeuf River near Lake } \\
\text { Village, Ark. }\end{array}$ & $355^{e}$ & 35 & 4,530 & 6,940 & 8,590 & 10,700 & 12,300 & 13,900 \\
\hline 7367670 & $\begin{array}{l}\text { Wards Bayou tributary at } \\
\text { Montrose, Ark. }\end{array}$ & 3.24 & 23 & 262 & 383 & 464 & 567 & 643 & 719 \\
\hline $\begin{array}{l}7367680 \\
7367700\end{array}$ & $\begin{array}{l}\text { Boeuf River near Eudora, Ark. } \\
\text { Boeuf River near Ark.-La. } \\
\text { State line }\end{array}$ & $\begin{array}{l}640^{e} \\
785^{e}\end{array}$ & $\begin{array}{l}42 \\
35\end{array}$ & $\begin{array}{r}9,260 \\
11,700\end{array}$ & $\begin{array}{l}12,700 \\
15,300\end{array}$ & $\begin{array}{l}15,000 \\
17,400\end{array}$ & $\begin{array}{l}17,700 \\
19,800\end{array}$ & $\begin{array}{l}19,700 \\
21,400\end{array}$ & $\begin{array}{l}21,600 \\
22,800\end{array}$ \\
\hline $\begin{array}{l}7369680 \\
7369700\end{array}$ & $\begin{array}{l}\text { Bayou Macon at Eudora, Ark. } \\
\text { Bayou Macon near Rilbourne, } \\
\text { Ark. }\end{array}$ & $\begin{array}{l}485^{e} \\
504^{e}\end{array}$ & $\begin{array}{l}44 \\
11\end{array}$ & $\begin{array}{l}2,840 \\
2,800\end{array}$ & $\begin{array}{l}3,770 \\
4,120\end{array}$ & $\begin{array}{l}4,330 \\
4,930\end{array}$ & $\begin{array}{l}4,980 \\
5,880\end{array}$ & $\begin{array}{l}5,420 \\
6,530\end{array}$ & $\begin{array}{l}5,830 \\
7,140\end{array}$ \\
\hline
\end{tabular}

a Discharge in thousands of cublc feet per second.

b St. Francis floodway near Marked Tree (07047000) and St. Francis River at Marked Tree (07047500) combined.

c St. Francis River at Parkin (07047800) and St. Prancis Bay at Riverfront (07047900) combined.

d Adjusted to 45-year period based on data at stations 07258000 and 07263450 .

e Interchange of flow at high stages. 
Basin and Climatic Characteristics

The following are defined for use in this report:

1. Drainage area, A.--The contributing drainage area of the basin, in $\mathrm{mi}^{2}$.

2. Channel slope, S.--The slope, in feet per mile $(\mathrm{ft} / \mathrm{mi})$, computed between two points along the main channel--one point at 10 percent of the channel length, and the other point at 85 percent of the channel length. Both points are measured from the gaged site (or point of intersection when computing discharges for ungaged sites).

3. Main channel length, L.--The channel length, in miles, between the gaged site and the basin divide.

4. Mean basin elevation, E.--The average ground elevation, in $\mathrm{ft}$ above sea level, of several equal-area subdivisions of the basin. The drainage basin was overlain with an appropriate sized grid to provide a minimum of 25 subdivisions.

5. Mean annual precipitation, P.--The mean annual precipitation for the basin, in in., during the period 1951-81 (see fig. 3) (Freiwald, 1985).

\section{Regression Analysis}

After the discharge-frequency relations were defined and basin and climatic parameters determined for gaging stations used in the analysis, the peak discharge for each recurrence interval was related to the basin and climatic parameters using the linear multiple-regression techniques. The equation has the form:

$$
\mathrm{Y}=\mathrm{aA}^{\mathrm{bl}} \mathrm{S}^{\mathrm{b} 2} \mathrm{p}^{\mathrm{b} 3}---
$$

where,

$\mathrm{Y}$ is the peak-discharge characteristic,

$A, S$, and $P$ are basin and climatic characteristics, and

$a, b 1, b 2$, and b3 are constants and coefficients obtained by regression analysis.

Initially all basin and climati: parameters were used in each regression. The final regression used only those that were statistically significant at the 5-percent level. Only stations with drainage basins less than 3,000 $\mathrm{mi}^{2}$ having predominantly natural flow and at least 10 years of record were used in the regression analysis. On regulated streams, the data collected prior to regulation were used if that period, was of sufficient length.

Initially, data for all stations in the State were used in one regression analysis and the residuals were plotted on a State map. The residual is the actual discharge from station data divided by the computed discharge using the regression equation. The residual from the regression analysis was plotted on a State map. The distribution of residuals showed that the state should be divided into two regions to improve the accuracy of the regression equations 
because residuals were generally positive in one region and negative in the other. These regions are the same as those defined by Patterson (1971) in the previous flood-frequency report. Region A (fig. 4) includes most of the Mississippi Alluvial Plain in Arkansas, with the exception of Crowleys Ridge. Region $B$ includes the rest of the State. Equations were developed separately for Regions $A$ and for $B$.

Each basin characteristic was checked by residual analysis to see if it was biased. Biased values did occur for slopes greater than $30 \mathrm{ft} / \mathrm{mi}$ and for mean basin elevations greater than $500 \mathrm{ft}$. To remove this bias, all slopes greater than $30 \mathrm{ft} / \mathrm{mi}$ were used as $30 \mathrm{ft} / \mathrm{mi}$ and all elevations higher than $500 \mathrm{ft}$ were used as $500 \mathrm{ft}$.

\section{Peak Discharge Equations for Region A}

The regression analysis indicated that, drainage area, channel slope, and channel length are significant characteristics for estimating flood magnitudes in Region $A$. The following equations were developed for rural streams by the multiple regression technique using data from 33 gaging stations in Region A (fig. 4) in Arkansas. Data for these stations are listed in table 1.

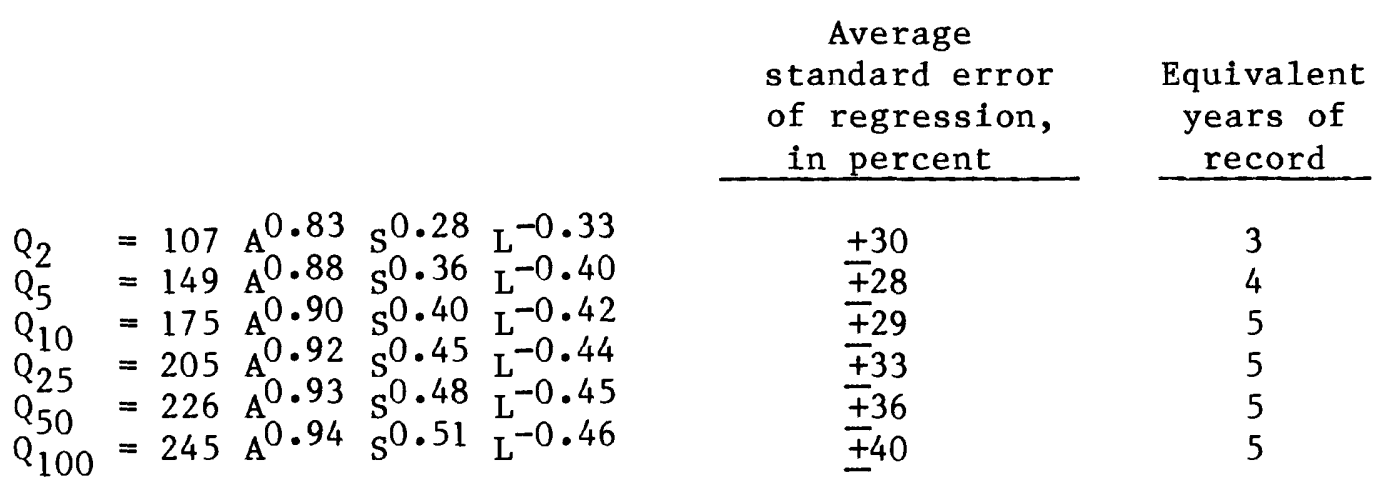

where,

$Q_{x}=$ the estimated discharge, in $\mathrm{ft}^{3} / \mathrm{s}$ for the indicated recurrence interval $\mathrm{x}$,

$\mathrm{A}=$ drainage area, in $\mathrm{mi}^{2}$,

$\mathrm{S}=$ channel slope, in $\mathrm{ft} / \mathrm{mi}$. If $\mathrm{S}$ is greater than 30 , use 30 for $\mathrm{S}$, and

$\mathrm{L}=$ channel length, in miles.

Channel length, $L$, is used in the equations as an index to shape and has a negative exponent. Two basins of equal area will have a lower discharge in the longer basin because of the longer travel time.

Landers (1985) developed equations for the alluvial plain of the lower Mississippi River which includes Region A in Arkansas. Computed discharges using Lander's equations compare favorably with computed discharges using equations shown above. 
Peak Discharge Equations for Region B

The regression analysis indicated that drainage area, channel slope, annual precipitation, and mean basin elevation are significant parameters for estimating flood magnitudes in Region B. The following equations were developed using data from 167 gaging stations and are recommended for estimating flood magnitudes for rural streams in Region B (fig. 4). Data for these stations are listed in table 2 .

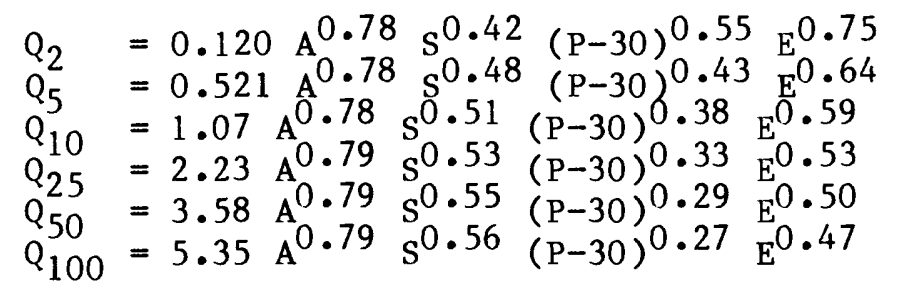

\begin{tabular}{lc}
$\begin{array}{l}\text { Average } \\
\text { standard } \\
\text { error of } \\
\text { regression, }\end{array}$ & $\begin{array}{l}\text { Equivalent } \\
\text { years of } \\
\text { in percent }\end{array}$ \\
\cline { 1 - 2 } record \\
\cline { 1 - 2 }+42 & 4 \\
\pm 34 & 7 \\
\pm 33 & 10 \\
\pm 33 & 13 \\
\pm 35 & 14 \\
\pm 38 & 14
\end{tabular}

where,

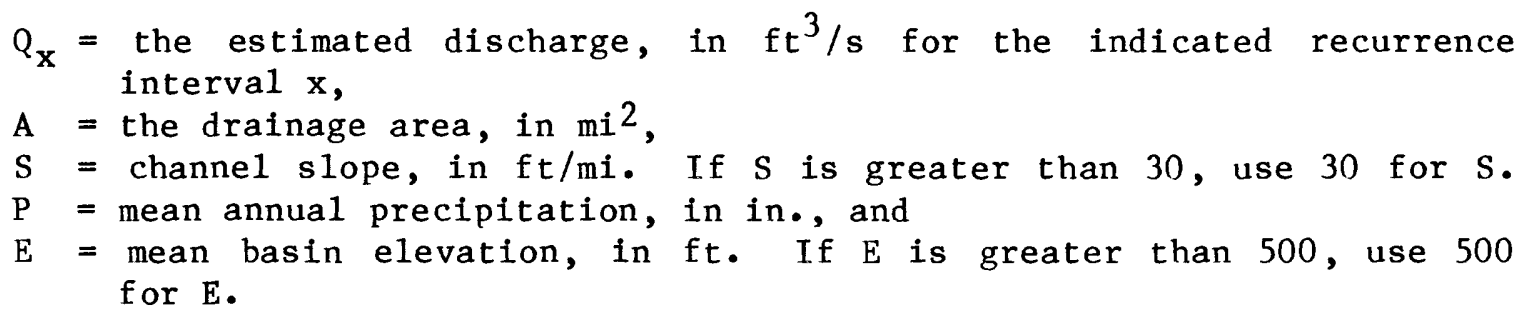

\section{Accuracy of Estimating Equations}

Accuracy of estimating equations is computed from the difference between station data and the regression equations. The accuracy, in percent, referred to as standard error, is the range of error to be expected about two-thirds of the time. The actual error associated with the use of the equations to estimate flood magnitudes at a given ungaged site is unknown. The error is assumed to equal the standard error of the regression equation.

The accuracy of estimating equations can also be evaluated by the equivalent years of record. The equivalent years of record are an estimate of the number of actual years of streamflow record required at a site to achieve an accuracy equivalent to the regional flood estimate from regression equations (Hardison, 1969). It is used in this report to weight the station log-Pearson Type III data with the regression flood-frequency estimate. This weighting procedure is intended to produce a flood-frequency relation at each site that is more realistic of the long-term relation than the station data or the regression data alone. This procedure for weighting the data is described in a later section of this report. 
Sensitivity analyses were performed to measure the sensitivity of the regression equation to measurement errors of the regression parameters. All parameters were assumed to be constant except the one being tested for sensitivity; that parameter was assumed to contain an error ranging from +50 percent to -50 percent. For example, assume that drainage area, A, contains an error of +30 percent in Region $A$. Then the effect on computed 2-year peak discharge would be 24 percent. The sensitivity of the estimating equations for the 2- and 100-year flood magnitudes to error in basin and climatic parameters is shown below.

Percent error in computed discharge for Region A

\begin{tabular}{crrrrrr}
$\begin{array}{c}\text { Percent error } \\
\text { in independent } \\
\text { variable }\end{array}$ & \multicolumn{2}{c}{$\begin{array}{c}\text { 2-year discharge } \\
\text { independent variable }\end{array}$} & \multicolumn{3}{c}{$\begin{array}{c}\text { 100-year discharge } \\
\text { independent variable }\end{array}$} \\
\cline { 2 - 7 } & A & S & L & & & A \\
\hline 50 & 40 & 12 & -13 & 46 & 23 & -18 \\
30 & 24 & 8 & -8 & 28 & 14 & -12 \\
10 & 8 & 3 & -3 & 9 & 5 & -4 \\
-10 & -8 & -3 & 4 & -9 & -5 & 5 \\
-30 & -26 & -10 & 12 & -28 & -17 & 18 \\
-50 & -44 & -18 & 26 & -48 & -30 & 39 \\
\hline
\end{tabular}

Percent error in computed discharge for Region B

\begin{tabular}{|c|c|c|c|c|c|c|c|c|}
\hline \multirow{2}{*}{$\begin{array}{l}\text { Percent error } \\
\text { in independent } \\
\text { variable } \\
\end{array}$} & \multicolumn{4}{|c|}{$\begin{array}{c}\text { 2-year discharge } \\
\text { independent variable }\end{array}$} & \multicolumn{4}{|c|}{$\begin{array}{l}\text { 100-year discharge } \\
\text { independent variable }\end{array}$} \\
\hline & $\bar{A}$ & $\mathrm{~S}$ & $\mathrm{P}$ & $\mathrm{E}$ & $\overline{\mathrm{A}}$ & $\mathrm{S}$ & $\mathrm{P}$ & $\mathrm{E}$ \\
\hline 50 & 37 & 19 & 25 & 36 & 38 & 25 & 12 & 21 \\
\hline 30 & 22 & 12 & 16 & 22 & 23 & 16 & 7 & 13 \\
\hline 10 & 8 & 4 & 5 & 7 & 8 & 5 & 3 & 5 \\
\hline-10 & -8 & -4 & -6 & -8 & -8 & -6 & -3 & -5 \\
\hline-30 & -24 & -14 & -18 & -23 & -25 & -18 & -9 & -15 \\
\hline-50 & -41 & -25 & -32 & -41 & -42 & -32 & -17 & -28 \\
\hline
\end{tabular}

It is very important to accurately measure the parameters that are used in the regression equation. The percent error tables for Regions $A$ and $B$ show that the equations are most sensitive to errors in drainage area, $A$.

\section{Limitations}

The following limitations should be observed when using the regression equations.

1. They should be used for rural areas of Arkansas and should not be used in urban areas unless the effects of urbanization are insignificant. 
2. They should not be used where dams, flood-detention structures, and other manmade works have a significant effect on peak discharges. Under such conditions, stream-system studies beyond the scope of this report involving reservoir and open-channel routing may be required to evaluate flood frequency. $\mathrm{mi}^{2}$.

3. They should be used on streams with drainage areas less than 3,000

\section{FLOOD MAGNITUDE AND FREQUENCY AT OR NEAR GAGED SITES ON STREAMS DRAINING LESS THAN 3,000 SQUARE MILES}

Flood frequency at gaged sites less than $3,000 \mathrm{mi}^{2}$ can be determined by a combined use of the regression equations and the gaging station frequency curve. The recommended procedure is to compute the discharge for the desired recurrence interval as a weighted average of the station value and the regression value. The weighted average is based on the length of record of the station data and equivalent years of record for the regression value as determined in a previous section. The weighted values are considered the best estimates for design purposes at a site. The equation used to compute the weighted average is

$$
Q x(w)=\frac{Q x(s)(N)+Q x(r)(E Q)}{N+E Q}
$$

where,

$$
\begin{aligned}
& \mathrm{Qx}(\mathrm{w})=\text { the weighted discharge for recurrence interval } \mathrm{x}, \\
& \mathrm{Qx}(\mathrm{s})=\text { the station discharge for recurrence interval } \mathrm{x}, \\
& \mathrm{Qx}(\mathrm{r})=\text { the regression discharge for recurrence interval } \mathrm{x}, \\
& \mathrm{N}=\text { the number of years of station data used to compute } \mathrm{Qx}(\mathrm{s}) \text {, and } \\
& \mathrm{EQ} \quad=\text { the equivalent years of record for } \mathrm{Qx}(\mathrm{r}) \text { shown previously. }
\end{aligned}
$$

\begin{tabular}{|c|c|c|c|c|c|c|c|}
\hline $\begin{array}{l}\text { Recurrence } \\
\text { interva1 } x, \\
\text { in years }\end{array}$ & $\begin{array}{l}\mathrm{Qx}(\mathrm{s}), \\
\text { in } \mathrm{ft} 3 / \mathrm{s} \\
\end{array}$ & in & $\begin{array}{l}\mathrm{N}, \\
\text { years }\end{array}$ & $\begin{array}{c}\mathrm{Qx}(\mathrm{r}), \\
\text { in } \mathrm{ft} \mathrm{t}^{3} / \mathrm{s} \\
\end{array}$ & in & $\begin{array}{l}\mathrm{E}, \\
\text { years }\end{array}$ & $\begin{array}{r}Q x(w), 1 \\
\text { in } f t^{3} / s \\
\end{array}$ \\
\hline 2 & 9,130 & & 41 & 7,670 & & 4 & 9,000 \\
\hline 5 & 14,800 & & 41 & 13,700 & & 7 & 14,600 \\
\hline 10 & 19,000 & & 41 & 19,000 & & 10 & 19,000 \\
\hline 25 & 24,600 & & 41 & 26,100 & & 13 & 25,000 \\
\hline 50 & 29,000 & & 41 & 32,500 & & 14 & 29,900 \\
\hline 100 & 33,600 & & 41 & 38,900 & & 14 & 35,000 \\
\hline
\end{tabular}

An example of the computations is illustrated in the following table by using station 07073000 , Strawberry River near Evening Shade.

$1 \mathrm{Qx}(\mathrm{w})$ values are shown in tables 1 and 2 for all gaging stations used to define the regression equations. 
Flood frequency at sites less than $3,000 \mathrm{mi}^{2}$ that are relatively near a gaging station and on the same stream can be calculated by a combined use of the regression equations and the nearby station data. The following procedure is suggested for use if the site has a drainage area within 50 percent of the drainage area of the gaging station. The weighted value, $Q x(w)$, and the regression value, $Q x(r)$, for the gaged site should be computed as described in the preceding paragraphs.

The ratio,

$$
R=\frac{Q x(w)}{Q x(r)}
$$

is then calculated for the gaged site. This ratio represents the correction needed to adjust the regression value, $Q x(r)$, to the weighted value, $Q x(w)$, at the gaged site. The calculations for determining the correction factor, $R^{\prime}$, for an ungaged site that is near a gaged site on the same stream have been reduced to the equation

$$
R^{\prime}=R-\frac{\Delta A(R-1.00)}{0.5 A g}
$$

where,

$$
\begin{aligned}
& \mathrm{R}^{\prime}=\text { correction factor that is multiplied by the regression value, } \\
& \text { Qx }(\mathrm{r}) \text {, for the ungaged site, } \\
& \Delta \mathrm{A}=\text { the difference between the drainage areas of the gaged and } \\
& \text { ungaged sites, and } \\
& \mathrm{Ag}=\text { drainage area of the gaged site. }
\end{aligned}
$$

If the drainage area of the ungaged site is 50 percent more than or less than the gaged site, that is, $\triangle \mathrm{A} / \mathrm{Ag}$ is greater than 0.5 , equation 16 should not be used and the results of the regression equations should be used without adjustment.

The site for which flood-frequency calculations are desired may sometimes be between two gaged sites on the same stream. The 50-percent rule should be applied to determine which gage, if any, should be used to make the adjustment. If the ungaged site is within 50 percent of both gaged sites, correction factors should be computed using each gaged site. If both correction factors are greater than unity, the larger should be used. If both correction factors are less than unity, the smaller should be used. If one is greater than unity and one is less than unity, an average of both correction factors should be used.

The following example illustrates the calculations for determining a 100-year flood for an ungaged site that is between two gaging stations on the same stream. For this example the gaged sites are station 07073000, Strawberry River near Evening Shade, Arkansas, and station 07074000 , Strawberry River near Poughkeepsie, Arkansas; and the ungaged site is where the drainage area equals $300 \mathrm{mi}^{2}$. Following are data and calculations needed for the gaged and ungaged sites, which are used to compute $Q_{100}$ at the ungaged 
site. The drainage area of the ungaged site $\left(300 \mathrm{mi}^{2}\right)$ is within 50 percent of the drainage areas at both gaged sites. Therefore, the station data for both gaged sites are used in the computations.

(1) Gaged site, 07073000, Strawberry River near Evening Shade:

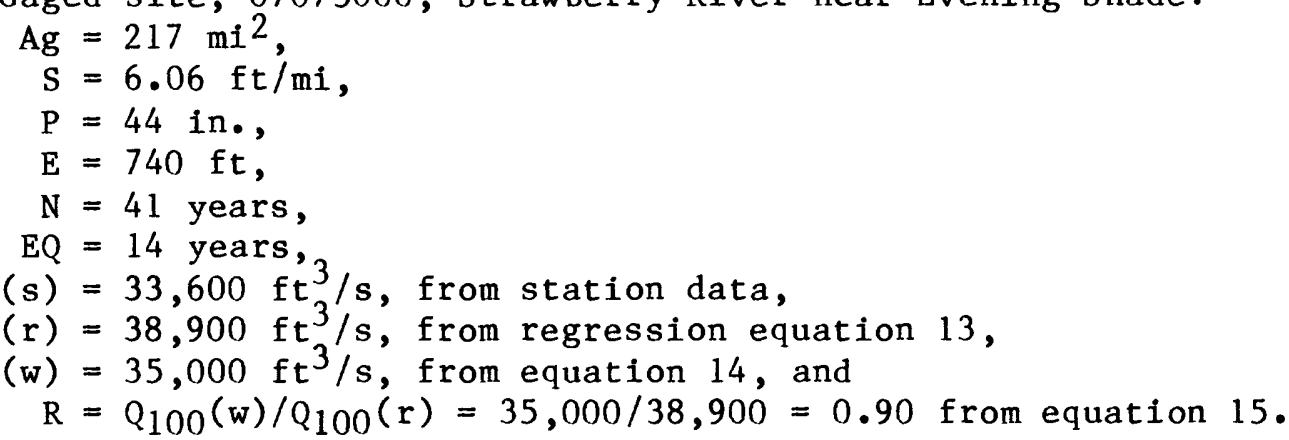

(2) Gaged site, 07074000 , Strawberry River near Poughkeepsie:

$\mathrm{Ag}=473 \mathrm{mi}^{2}$,

$\mathrm{S}=5.96 \mathrm{ft} / \mathrm{mi}$,

$\mathrm{P}=45$ in.,

$\mathrm{E}=680 \mathrm{ft}$,

$\mathrm{N}=48$ years,

$\mathrm{EQ}=14$ years

$Q_{100}(\mathrm{~s})=87,600 \mathrm{ft}^{3} / \mathrm{s}$, from station data,

$\mathrm{Q}_{100}(\mathrm{r})=72,800 \mathrm{ft}^{3} / \mathrm{s}$, from regression equation 13 ,

$\mathrm{Q}_{100}(\mathrm{w})=84,200 \mathrm{ft}^{3} / \mathrm{s}$, from equation 14 , $R=Q_{100}(w) / Q_{100}(r)=84,200 / 72,800=1.16$ from equation 15 .

(3) Ungaged site:

$$
\begin{aligned}
\mathrm{A} & =300 \mathrm{mi}^{2}, \\
\mathrm{~S} & =6.00 \mathrm{ft} / \mathrm{mi}, \\
\mathrm{P} & =45 \mathrm{in} ., \\
\mathrm{E} & =710 \mathrm{ft}, \\
\mathrm{Q}_{100}(\mathrm{r}) & =51,000 \mathrm{ft}^{3} / \mathrm{s}, \text { from regression equation } 13 \\
\Delta \mathrm{A} & =300-217=83 \mathrm{mi} \text { using Evening Shade } \\
\mathrm{R}^{\prime} & =0.90-\frac{83}{0.5 * 217} \quad(0.90-1.00)=0.98 \text { using Evening Shade } \\
\Delta \mathrm{A} & =473-300=173 \mathrm{mi}^{2} \text { using Poughkeepsie } \\
\mathrm{R}^{\prime} & =1.16-\frac{173}{0.5 * 473} \quad(1.16-1.00)=1.04 \text { using Poughkeepsie. }
\end{aligned}
$$

Since $R^{\prime}$ using Evening Shade is less than unity and $R^{\prime}$ using Poughkeepsie is greater than unity, the average is used.

$$
\begin{aligned}
R^{\prime} & =\left(0.98+1 . Q^{4}\right) / 2=1.01 \\
Q_{100} & =Q_{100}(r)(R)=51,000(1.01)=51,500 \mathrm{ft}^{3} / \mathrm{s} .
\end{aligned}
$$

This is considered the best estimate for the 100-year peak discharge at the ungaged site on Strawberry River. 
FLOOD MAGNITUDE AND FREQUENCY AT UNGAGED SITES ON STREAMS DRAINING LESS THAN 3,000 SQUARE MILES USING THE HYDRAULIC RADIUS

An alternate procedure for computing flood frequency which uses the hydraulic radius as one of the basin characteristics is described in this section. The hydraulic radius in conjunction with the channel slope probably is a better index to the velocity of the flood wave than channel slope alone. Hydraulic radius has not been used in flood-frequency reports in the past because it is difficult to determine at ungaged sites. In order to determine the hydraulic radius at an ungaged site, a cross section of the stream valley and a stage-discharge relation must be known. This part of the report describes a way to estimate a cross section from topographic maps and to compute a stage-discharge relation for that section. The hydraulic radius and the corresponding discharge can be determined at several stages to develop a hydraulic radius-discharge relation. The hydraulic radius can then be determined for any discharge for use in equations 17-28.

Using this procedure a preliminary discharge for any selected recurrence interval can be determined using equations 2-13. This preliminary discharge is used to determine the hydraulic radius at the ungaged site. The hydraulic radius and other basin and climatic characteristics are used in equations 17-28 to determine the final discharge. Calculations of the hydraulic radius are not recomputed using the results of equations 17-28.

The peak discharge equations for Region A using the hydraulic radius procedure are shown below. The hydraulic radius and $\mathrm{N}$ data for these stations are 1 isted in table 4.

Average

standard error of regression, in percent

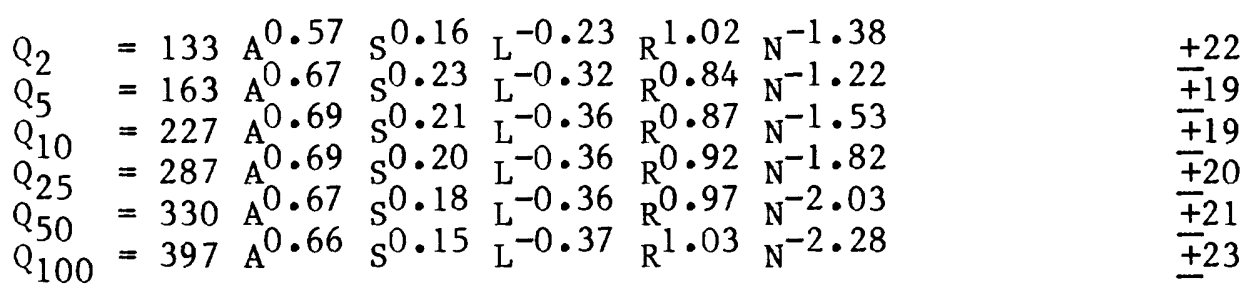

The peak discharge equations for Region $B$ using the hydraulic radius procedure are shown below. The hydraulic radius and $\mathrm{N}$ data for these stations are listed in table 5 .

$$
\begin{array}{lllllllll}
\mathrm{Q}_{2}= & =5.24 & \mathrm{~A}^{0.45} & \mathrm{~S}^{0.23} & (\mathrm{P}-30)^{0.20} & \mathrm{E}^{0.36} & \mathrm{R}^{1.21} & \mathrm{~N}^{-1.12} & \pm 30 \\
\mathrm{Q}_{5}=7.20 & \mathrm{~A}^{0.51} & \mathrm{~S}^{0.29} & (\mathrm{P}-30)^{0.24} & \mathrm{E}^{0.32} & \mathrm{R}^{0.99} & \mathrm{~N}^{-0.90} & \pm 28 \\
\mathrm{Q}_{10}=10.9 & \mathrm{~A}^{0.53} & \mathrm{~S}^{0.32} & (\mathrm{P}-30)^{0.24} & \mathrm{E}^{0.28} & \mathrm{R}^{0.92} & \mathrm{~N}^{-0.84} & \pm 27 \\
\mathrm{Q}_{25}=20.7 & \mathrm{~A}^{0.54} & \mathrm{~S}^{0.34} & (\mathrm{P}-30)^{0.21} & \mathrm{E}^{0.20} & \mathrm{R}^{0.93} & \mathrm{~N}^{-0.83} & \pm 28 \\
\mathrm{Q}_{50}=34.5 & \mathrm{~A}^{0.53} & \mathrm{~S}^{0.34} & (\mathrm{P}-30)^{0.19} & \mathrm{E}^{0.14} & \mathrm{R}^{0.97} & \mathrm{~N}^{-0.86} & \pm 28 \\
\mathrm{Q}_{100}=53.3 & \mathrm{~A}^{0.52} & \mathrm{~S}^{0.34} & (\mathrm{P}-30)^{0.17} & \mathrm{E}^{0.09} & \mathrm{R}^{1.01} & \mathrm{~N}^{-0.89} & \pm+20
\end{array}
$$




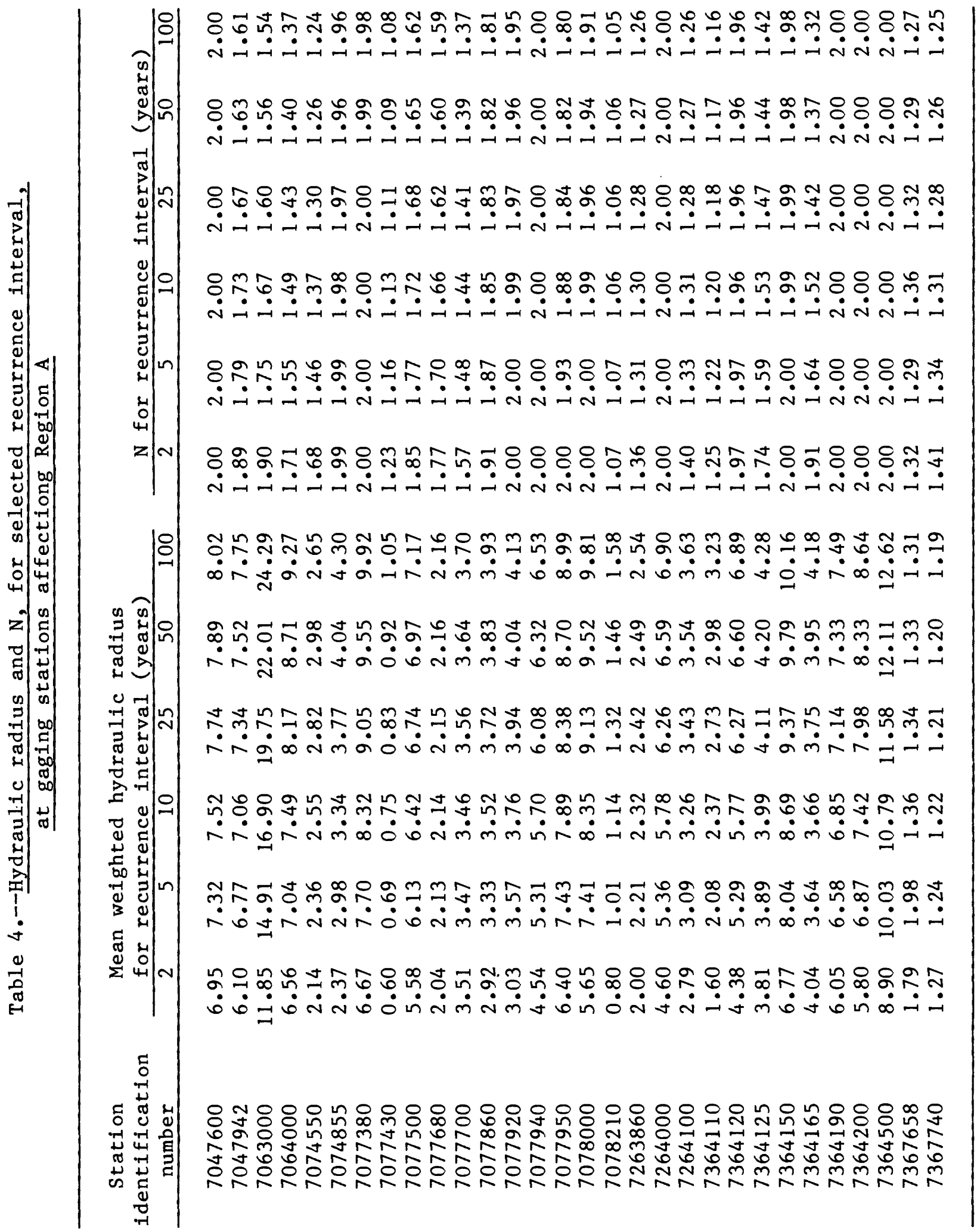


Table 5.-Hydraulic radius and $N$, for selected recurrence interval, at gaging stations affecting Region B

\begin{tabular}{|c|c|c|c|c|c|c|c|c|c|c|c|c|}
\hline \multirow{2}{*}{$\begin{array}{c}\text { Station } \\
\text { identification } \\
\text { number }\end{array}$} & \multicolumn{6}{|c|}{$\begin{array}{l}\text { Mean weighted hydraulic radius for } \\
\text { recurrence Interval (years) }\end{array}$} & $\mathrm{N}$ & for $r$ & urrenc & Inter & al (ye & rs) \\
\hline & 2 & 5 & 10 & 25 & 50 & 100 & 2 & 5 & 10 & 25 & 50 & 100 \\
\hline 7047820 & 2.22 & 2.13 & 2.12 & 2.21 & 2.27 & 2.33 & 1.65 & 1.45 & 1.38 & 1.31 & 1.27 & 1.24 \\
\hline 7047880 & 1.11 & 1.45 & 1.71 & 2.06 & 2.37 & 2.72 & 1.57 & 1.41 & 1.33 & 1.27 & 1.23 & 1.20 \\
\hline 7047975 & 2.13 & 2.55 & 2.83 & 3.20 & 3.47 & 3.75 & 1.86 & 1.67 & 1.59 & 1.50 & 1.45 & 1.42 \\
\hline 7047990 & 1.71 & 2.56 & 3.17 & 3.93 & 4.48 & 5.01 & 1.53 & 1.36 & 1.30 & 1.25 & 1.22 & 1.20 \\
\hline 7048000 & 5.81 & 7.61 & 9.08 & 10.72 & 11.82 & 12.83 & 2.00 & 2.00 & 1.99 & 1.98 & 1.96 & 1.94 \\
\hline 7048600 & 10.47 & 12.08 & 12.83 & 13.64 & 14.19 & 14.71 & 1.91 & 1.83 & 1.78 & 1.73 & 1.70 & 1.68 \\
\hline 7048900 & 1.92 & 2.47 & 2.84 & 3.33 & 3.79 & 4.31 & 1.56 & 1.45 & 1.39 & 1.33 & 1.29 & 1.26 \\
\hline 7048940 & 6.37 & 8.39 & 9.59 & 10.98 & 11.97 & 12.92 & 1.70 & 1.62 & 1.58 & 1.53 & 1.50 & 1.47 \\
\hline 7049000 & 10.65 & 14.18 & 16.25 & 18.60 & 20.16 & 21.60 & 1.96 & 1.93 & 1.92 & 1.90 & 1.89 & 1.88 \\
\hline 7049500 & 13.39 & 18.37 & 21.23 & 24.41 & 26.53 & 28.44 & 1.98 & 1.95 & 1.94 & 1.92 & 1.91 & 1.90 \\
\hline 7050000 & 10.46 & 12.92 & 14.41 & 16.15 & 17.37 & 18.54 & 2.00 & 2.00 & 2.00 & 2.00 & 2.00 & 2.00 \\
\hline 7050200 & 3.30 & 4.26 & 4.98 & 5.94 & 6.65 & 7.34 & 1.82 & 1.62 & 1.54 & 1.46 & 1.41 & 1.38 \\
\hline 7050400 & 1.84 & 2.12 & 2.31 & 2.55 & 2.72 & 2.88 & 1.64 & 1.50 & 1.44 & 1.39 & 1.36 & 1.33 \\
\hline 7050500 & 8.19 & 11.36 & 13.29 & 15.57 & 17.16 & 18.64 & 2.00 & 2.00 & 2.00 & 2.00 & 2.00 & 1.99 \\
\hline 7054400 & 3.81 & 4.49 & 5.10 & 5.95 & 6.62 & 7.29 & 1.70 & 1.53 & 1.46 & 1.39 & 1.35 & 1.32 \\
\hline 7054450 & 1.80 & 2.28 & 2.60 & 3.01 & 3.33 & 3.70 & 1.38 & I. 30 & 1.26 & 1.22 & 1.19 & 1.17 \\
\hline 7055550 & 3.43 & 3.72 & 3.95 & 4.28 & 4.56 & 4.85 & 1.90 & 1.71 & 1.61 & 1.51 & 1.45 & 1.40 \\
\hline 7055650 & 3.29 & 4.51 & 5.24 & 6.10 & 6.75 & 7.37 & 2.00 & 2.00 & 2.00 & 2.00 & 2.00 & 2.00 \\
\hline 7055800 & 4.50 & 6.05 & 6.94 & 7.99 & 8.74 & 9.49 & 1.90 & 1.84 & 1.80 & 1.75 & 1.71 & 1.67 \\
\hline 7056000 & 14.45 & 19.57 & 22.49 & 25.98 & 28.32 & 30.65 & 2.00 & 1.98 & 1.95 & 1.93 & 1.91 & 1.90 \\
\hline 7057000 & 13.93 & 17.46 & 19.34 & 21.54 & 23.14 & 24.62 & 2.00 & 2.00 & 2.00 & 2.00 & 2.00 & 2.00 \\
\hline 7057300 & 1.76 & 2.13 & 2.34 & 2.57 & 2.72 & 2.86 & 1.24 & 1.20 & 1.18 & 1.17 & 1.16 & 1.15 \\
\hline 7060600 & 2.24 & 2.39 & 2.58 & 2.88 & 3.12 & 3.39 & 1.89 & 1.68 & 1.57 & 1.47 & 1.41 & 1.36 \\
\hline 7060670 & 3.98 & 4.59 & 5.00 & 5.48 & 5.83 & 6.16 & 1.54 & 1.44 & 1.40 & 1.35 & 1.33 & 1.30 \\
\hline 7060710 & 4.84 & 7.71 & 9.72 & 12.40 & 14.39 & 16.36 & 2.00 & 2.00 & 2.00 & 2.00 & 2.00 & 2.00 \\
\hline 7060830 & 1.44 & 1.82 & 2.02 & 2.25 & 2.43 & 2.60 & 1.99 & 1.87 & 1.78 & 1.69 & 1.63 & 1.58 \\
\hline 7061100 & 3.86 & 4.45 & 4.56 & 4.60 & 4.68 & 4.83 & 1.79 & 1.63 & 1.58 & 1.51 & 1.45 & 1,41 \\
\hline 7068000 & 10.42 & 14.62 & 16.59 & 18.44 & 19.76 & 21.08 & 2.00 & 1.99 & 1.96 & 1.90 & 1.87 & 1.84 \\
\hline 7068870 & 1.36 & 1.57 & 1.69 & 1.84 & 1.95 & 2.05 & 1.30 & 1.24 & 1.21 & 1.19 & 1.17 & 1.16 \\
\hline 7068890 & 12.80 & 12.17 & 12.42 & 13.07 & 13.66 & 14.31 & 1.89 & 1.63 & 1.54 & 1.46 & 1.41 & 1.38 \\
\hline 7069250 & 2.00 & 2.50 & 2.83 & 3.24 & 3.56 & 3.88 & 1.41 & 1.30 & 1.25 & 1.21 & 1.18 & 1.16 \\
\hline 7069290 & 2.48 & 3.08 & 3.53 & 4.11 & 4.53 & 4.95 & 1.55 & 1.40 & 1.34 & 1.29 & 1.26 & 1.24 \\
\hline 7069500 & 12.16 & 16.92 & $19.4 \mathrm{I}$ & 22.08 & 23.88 & 25.43 & 2.00 & 1.97 & 1.93 & 1.87 & 1.83 & 1.79 \\
\hline 7071500 & 8.59 & 12.44 & 13.92 & 15.71 & 17.04 & 18.37 & 2.00 & 1.95 & 1.88 & 1.81 & 1.77 & 1.73 \\
\hline 7072000 & 10.03 & 14.26 & 16.37 & 18.25 & 19.30 & 20.53 & 1.99 & 1.98 & 1.93 & 1.85 & 1.77 & 1.71 \\
\hline 7072200 & 2.15 & 2.45 & 2.61 & 2.78 & 2.90 & 3.00 & 1.13 & 1.10 & I. .09 & 1.08 & 1.08 & 1.07 \\
\hline 7073000 & 8.39 & 10.86 & 12.42 & $14 \cdot 14$ & 15.27 & 16.31 & 2.00 & 2.00 & 2.00 & 1.99 & 1.98 & 1.97 \\
\hline 7073500 & 5.79 & 8.15 & 9.73 & 11.75 & 13.26 & 14.86 & 2.00 & 2.00 & 2.00 & 2.00 & 2.00 & 2.00 \\
\hline 7074000 & 9.95 & 13.24 & 15.75 & 18.61 & 20.49 & 22.36 & 2.00 & 2.00 & 1.99 & 1.96 & 1.94 & 1.91 \\
\hline 7074200 & 2.96 & 2.67 & 2.74 & 2.90 & 3.02 & 3.13 & 1.49 & 1.38 & 1.32 & 1.27 & 1.25 & 1.22 \\
\hline 7074250 & 6.11 & 7.55 & 8.08 & 8.56 & 8.88 & 9.22 & 1.76 & 1.67 & 1.61 & 1.53 & 1.47 & 1.42 \\
\hline 7074900 & 1.29 & 1.55 & 1.73 & 1.94 & 2.09 & 2.23 & 1.62 & 1.43 & 1.36 & 1.30 & 1.27 & 1.24 \\
\hline 7074950 & 2.04 & 2.68 & 3.20 & 3.86 & 4.30 & 4.69 & 1.69 & 1.44 & 1.36 & 1.29 & 1.26 & 1.23 \\
\hline 7075000 & 11.54 & 15.77 & 17.99 & 20.34 & 21.86 & 23.20 & 2.00 & 1.96 & 1.92 & 1.86 & 1.81 & 1.76 \\
\hline 7075300 & 14.21 & 16.98 & 18.41 & 19.95 & 20.96 & 21.92 & 1.89 & 1.83 & 1.80 & 1.77 & 1.74 & 1.72 \\
\hline 7075500 & 11.62 & 14.18 & 15.66 & 17.34 & 18.45 & 19.50 & 1.93 & 1.88 & 1.86 & 1.84 & 1.82 & 1.81 \\
\hline 7075600 & 2.32 & 2.76 & 3.08 & 3.53 & 3.91 & 4.36 & 1.82 & 1.65 & 1.57 & 1.48 & 1.42 & 1.37 \\
\hline 7075800 & 1.19 & 1.40 & 1.60 & 1.88 & 2.09 & 2.30 & 1.90 & 1.62 & 1.49 & 1.39 & 1.33 & 1.29 \\
\hline 7076000 & 16.46 & 18.78 & 19.67 & 20.61 & 21.26 & 21.81 & 2.00 & 2.00 & 2.00 & 2.00 & 2.00 & 2.00 \\
\hline 7076630 & 2.00 & 2.28 & 2.43 & 2.59 & 2.69 & 2.79 & 1.26 & 1.23 & 1.21 & 1.20 & 1.19 & 1.18 \\
\hline 7076820 & 3.03 & 3.01 & 3.00 & 3.00 & 3.00 & 3.00 & 1.85 & 1.72 & 1.66 & 1.60 & 1.57 & 1.54 \\
\hline 7076850 & 6.23 & 6.50 & 6.72 & 7.04 & 7.28 & 7.51 & 1.69 & 1.54 & 1.47 & 1.41 & 1.38 & 1.36 \\
\hline 7076870 & 3.36 & 3.94 & 4.34 & 4.76 & 4.90 & 5.06 & 1.41 & 1.31 & 1.27 & 1.23 & 1.22 & 1.20 \\
\hline 7077100 & 5.36 & 6.54 & 7.16 & 7.78 & 8.15 & 8.36 & 2.00 & 2.00 & 2.00 & 2.00 & 1.99 & 1.98 \\
\hline 7077200 & 2.10 & 2.59 & 2.87 & 3.19 & 3.41 & 3.61 & 2.00 & 2.00 & 2.00 & 2.00 & 2.00 & 2.00 \\
\hline 7077340 & 1.78 & 1.95 & 2.08 & 2.29 & 2.43 & 2.55 & 1.61 & 1.43 & 1.36 & 1.30 & 1.27 & 1.25 \\
\hline 7188900 & 0.66 & 1.12 & 1.42 & 1.79 & 2.04 & 2.05 & 2.00 & 2.00 & 2.00 & 2.00 & 2.00 & 2.00 \\
\hline 7191220 & 5.90 & 9.25 & 11.39 & 13.95 & 15.76 & 17.43 & 1.78 & 1.67 & 1.60 & 1.52 & 1.47 & 1.43 \\
\hline 7194890 & 3.44 & 5.72 & 7.30 & 9.31 & 10.81 & 12.33 & 2.00 & 2.00 & 2.00 & 2.00 & 2.00 & 2.00 \\
\hline 7195000 & 7.23 & 10.43 & 12.03 & 13.60 & 14.54 & 15.35 & 1.99 & 1.96 & 1.93 & 1.87 & 1.83 & 1.80 \\
\hline 7195200 & 1.34 & 1.77 & 2.08 & 2.49 & 2.80 & 3.10 & 1.77 & 1.53 & 1.43 & 1.34 & 1.30 & 1.26 \\
\hline 7195450 & 3.91 & 6.13 & 7.46 & 8.74 & 9.47 & 10.07 & 2.00 & 2.00 & 1.99 & 1.97 & 1.94 & 1.91 \\
\hline 7195500 & 10.67 & 14.59 & 16.84 & 19.41 & 21.12 & 22.69 & 2.00 & 2.00 & 2.00 & 2.00 & $2.0 \mathrm{~J}$ & 2.00 \\
\hline 7195800 & 2.29 & 4.27 & 5.90 & 8.26 & 9.76 & 11.10 & 2.00 & 2.00 & 2.00 & 1.99 & 1.94 & 1.87 \\
\hline 7196000 & 5.56 & 9.79 & 12.96 & 16.48 & 18.34 & 19.57 & 2.00 & 2.00 & 1.99 & 1.94 & 1.88 & 1.80 \\
\hline 7196900 & 9.04 & 11.85 & 12.99 & 14.06 & 14.72 & 15.30 & 1.99 & 1.89 & 1.82 & 1.75 & 1.70 & 1.66 \\
\hline 7247000 & 9.27 & 12.02 & 13.33 & 14.60 & 15.39 & 16.05 & 2.00 & 1.97 & 1.93 & 1.89 & 1.86 & 1.83 \\
\hline 7249300 & 6.19 & 9.20 & 11.16 & 13.08 & 14.16 & 15.03 & 2.00 & 2.00 & 1.99 & 1.95 & 1.91 & 1.86 \\
\hline 7249400 & 7.11 & 9.50 & 11.01 & 12.84 & 14.16 & 15.75 & 2.00 & 2.00 & 2.00 & 2.00 & 2.00 & 2.00 \\
\hline 7249500 & 6.24 & 8.81 & 10.49 & 12.40 & 13.66 & 14.78 & 2.00 & 2.00 & 1.99 & 1.97 & 1.94 & 1.91 \\
\hline 7249650 & 3.79 & 5.27 & 6.15 & 7.12 & 7.62 & 8.02 & 2.00 & 2.00 & 2.00 & 1.99 & 1.97 & 1.94 \\
\hline 7249950 & 1.08 & 1.55 & 2.02 & 2.72 & 3.30 & 3.92 & 1.94 & 1.61 & 1.44 & 1.31 & 1.25 & 1.20 \\
\hline 7250000 & 12.37 & 16.08 & 18.20 & 20.54 & 22.10 & 23.57 & 1.96 & & 1.89 & & 1.83 & 1.80 \\
\hline 7252000 & 9.23 & 12.88 & 14.99 & 17.35 & 18.89 & 20.31 & 1.99 & 1.98 & 1.98 & 1.97 & 1.97 & 1.97 \\
\hline 7252200 & 1.66 & 1.92 & 2.14 & 2.43 & 2.65 & 2.87 & 1.78 & 1.59 & 1.50 & 1.42 & 1.37 & 1.33 \\
\hline 7252500 & 2.42 & 3.08 & 3.44 & 3.84 & 4.11 & 4.34 & 1.25 & 1.20 & 1.19 & 1.17 & 1.16 & 1.15 \\
\hline 7254000 & 2.71 & 3.53 & 3.99 & 4.52 & 4.88 & 5.21 & 1.64 & 1.53 & 1.47 & 1.42 & 1.39 & 1.36 \\
\hline 7254500 & 3.56 & 3.91 & 4.12 & 4.38 & 4.56 & 4.74 & 1.78 & 1.63 & 1.57 & 1.51 & 1.47 & 1.44 \\
\hline 7255100 & 2.87 & 3.78 & 4.29 & 4.84 & 5.20 & 5.53 & 1.32 & 1.26 & 1.23 & 1.20 & 1.19 & 1.18 \\
\hline 7256000 & 5.99 & 7.54 & 8.30 & 9.07 & 9.55 & 9.96 & 2.00 & 1.97 & 1.95 & 1.92 & 1.90 & 1.88 \\
\hline
\end{tabular}


Table 5. -Hydraulic radius and $N_{2}$ for selected recurrence interval, at gaging stations affecting Region B-Continued

\begin{tabular}{|c|c|c|c|c|c|c|c|c|c|c|c|c|}
\hline $\begin{array}{c}\text { Station } \\
\text { identification }\end{array}$ & & $\begin{array}{l}\text { ean we } \\
\text { recu }\end{array}$ & $\begin{array}{l}\text { shted } h \\
\text { cence } 1\end{array}$ & raul1 & $\begin{array}{l}\text { radiu } \\
\text { (years }\end{array}$ & for & $\mathbf{N}$ & for re & arrenc & Inter & al (ye & rs) \\
\hline & 2 & 5 & 10 & 25 & 50 & 100 & 2 & 5 & 10 & 25 & 50 & 100 \\
\hline 7256500 & 6.31 & 8.90 & 10.07 & 11.14 & 11.78 & 12.32 & 2.00 & 1.97 & 1.93 & 1.86 & 1.82 & 1.78 \\
\hline 7257000 & 9.09 & 12.77 & 15.13 & 18.01 & 20.07 & 22.02 & 1.95 & 1.93 & 1.92 & 1.90 & 1.89 & 1.88 \\
\hline 7257060 & 1.26 & 1.56 & 1.74 & 1.97 & 2.13 & 2.29 & 1.59 & 1.50 & 1.45 & 1.40 & 1.37 & 1.34 \\
\hline 7257100 & 1.09 & 1.23 & 1.34 & 1.58 & 1.77 & 1.95 & 1.86 & 1.56 & 1.44 & 1.35 & 1.30 & 1.26 \\
\hline 7257500 & 8.66 & 11.84 & 13.84 & 16.24 & 17.93 & 19.53 & 1.97 & 1.93 & 1.91 & 1.87 & 1.85 & 1.83 \\
\hline 7257700 & 3.74 & 4.54 & 5.00 & 5.63 & 6.27 & 6.96 & 1.94 & 1.72 & 1.60 & 1.49 & 1.42 & 1.37 \\
\hline 7258200 & 1.64 & 1.97 & 2.18 & 2.43 & 2.61 & 2.79 & 1.40 & 1.33 & 1.30 & 1.27 & 1.25 & 1.23 \\
\hline 7258500 & 8.34 & 10.88 & 12.10 & 13.33 & 14.08 & 14.73 & 2.00 & 1.97 & 1.94 & 1.90 & 1.87 & 1.84 \\
\hline 7260000 & 6.39 & 8.13 & 9.04 & 9.99 & 10.59 & 11.13 & 1.99 & 1.96 & 1.93 & 1.89 & 1.87 & 1.84 \\
\hline 7260630 & 2.35 & 2.67 & 2.94 & 3.27 & 3.51 & 3.74 & 1.59 & 1.40 & 1.33 & 1.27 & 1.24 & 1.22 \\
\hline 7260679 & 0.61 & 0.74 & 0.81 & 0.90 & 0.96 & 1.02 & 1.11 & 1.09 & 1.09 & 1.08 & 1.08 & 1.07 \\
\hline 7261000 & 7.09 & 9.23 & 10.42 & 11.72 & 12.59 & 13.38 & 2.00 & 2.00 & 2.00 & 2.00 & 2.00 & 2.00 \\
\hline 7261050 & 1.47 & 1.85 & 2.12 & 2.46 & 2.70 & 2.95 & 1.51 & 1.36 & 1.29 & 1.24 & 1.21 & 1.18 \\
\hline 7261300 & 2.73 & 3.59 & 4.26 & 5.22 & 5.99 & 6.84 & 1.78 & 1.56 & 1.46 & 1.36 & 1.31 & 1.26 \\
\hline 7261500 & 11.92 & 15.81 & 16.91 & 17.59 & 17.84 & 18.24 & 2.00 & 1.96 & 1.89 & 1.79 & 1.72 & 1.66 \\
\hline 7261800 & 2.12 & 2.57 & 2.90 & 3.36 & 3.71 & 4.09 & 1.72 & 1.53 & 1.45 & 1.36 & 1.32 & 1.28 \\
\hline 7263000 & 11.26 & 14.13 & 15.80 & 17.73 & 19.04 & 20.25 & 1.95 & 1.91 & 1.89 & 1.87 & 1.86 & 1.85 \\
\hline 7263100 & 2.04 & 2.33 & 2.53 & 2.77 & 2.95 & 3.11 & 1.59 & 1.45 & 1.39 & 1.34 & 1.31 & 1.28 \\
\hline 7263400 & 4.06 & 5.71 & 6.69 & 7.83 & 8.69 & 9.54 & 2.00 & 2.00 & 2.00 & 2.00 & 2.00 & 1.99 \\
\hline 7263910 & 2.33 & 2.60 & 2.76 & 2.94 & 3.06 & 3.18 & 1.30 & 1.26 & 1.25 & 1.23 & 1.22 & 1.21 \\
\hline 7338700 & 4.78 & 5.90 & 6.59 & 7.42 & 8.02 & 8.61 & 1.66 & 1.57 & 1.53 & 1.48 & 1.45 & 1.42 \\
\hline 7339500 & 8.90 & 11.91 & 14.01 & 15.36 & 15.88 & 16.22 & 2.00 & 2.00 & 1.97 & 1.88 & 1.81 & 1.73 \\
\hline 7339800 & 3.97 & 4.66 & 5.06 & 5.62 & 6.04 & 6.63 & 1.63 & 1.48 & 1.39 & 1.31 & 1.27 & 1.23 \\
\hline 7340000 & 16.98 & 17.44 & 17.43 & 17.68 & 18.02 & 18.46 & 1.99 & 1.85 & 1.76 & 1.68 & 1.63 & 1.59 \\
\hline 7340200 & 3.56 & 3.69 & 3.80 & 3.97 & 4.09 & 4.21 & 1.56 & 1.41 & 1.36 & 1.31 & 1.29 & 1.27 \\
\hline 7340300 & 11.14 & 15.28 & 17.60 & 20.17 & 21.85 & 23.37 & 1.93 & 1.90 & 1.88 & 1.86 & 1.84 & 1.83 \\
\hline 7340500 & 10.78 & 12.75 & 13.32 & 13.74 & 14.17 & 14.67 & 1.99 & 1.91 & 1.84 & 1.75 & 1.69 & 1.65 \\
\hline 7340530 & 2.12 & 2.79 & 2.98 & 3.07 & 3.19 & 3.33 & 1.34 & 1.29 & 1.28 & 1.26 & 1.24 & 1.23 \\
\hline 7341000 & 7.86 & 10.84 & 12.85 & 15.39 & 16.66 & 17.04 & 1.93 & 1.89 & 1.86 & 1.83 & 1.79 & 1.73 \\
\hline 7341100 & 3.88 & 4.67 & 5.20 & 5.90 & 6.53 & 7.18 & 1.47 & 1.32 & 1.27 & 1.22 & 1.20 & 1.18 \\
\hline 7341200 & 7.49 & 8.12 & 9.08 & 10.67 & 12.02 & 13.40 & 1.54 & 1.36 & 1.28 & 1.23 & 1.20 & 1.18 \\
\hline 7341700 & 3.95 & 4.45 & 4.82 & 5.29 & 5.62 & 5.94 & 1.48 & 1.36 & 1.31 & 1.27 & 1.24 & 1.22 \\
\hline 7342350 & 5.46 & 4.83 & 4.52 & 4.37 & 4.35 & 4.34 & 1.90 & 1.68 & 1.58 & 1.50 & 1.46 & 1.42 \\
\hline 7344320 & 2.38 & 2.44 & 2.55 & 2.71 & 2.83 & 2.95 & 1.83 & 1.58 & 1.48 & 1.41 & 1.37 & 1.34 \\
\hline 7346800 & 0.81 & 1.03 & 1.20 & 1.41 & 1.57 & 1.72 & 1.64 & 1.44 & 1.36 & 1.29 & 1.25 & 1.22 \\
\hline 7347000 & 4.08 & 4.87 & 5.34 & 5.88 & 6.27 & 6.63 & 2.00 & 2.00 & 2.00 & 2.00 & 2.00 & 2.00 \\
\hline 7348630 & 0.66 & 0.82 & 0.92 & 1.05 & 1.15 & 1.24 & 1.26 & 1.20 & 1.18 & 1.15 & 1.14 & 1.12 \\
\hline 7348700 & 7.82 & 10.11 & 10.49 & 11.26 & 12.03 & 12.91 & 2.00 & 1.90 & 1.77 & 1.66 & 1.59 & 1.54 \\
\hline 7349430 & 2.99 & 3.85 & 4.37 & 4.98 & 5.41 & 5.80 & 1.03 & 1.02 & 1.02 & 1.02 & 1.01 & 1.01 \\
\hline 7349500 & 6.58 & 9.00 & 10.44 & 11.67 & 12.33 & 12.83 & 2.00 & 2.00 & 2.00 & 1.97 & 1.94 & 1.91 \\
\hline 7355800 & 1.60 & 1.94 & 2.15 & 2.40 & 2.58 & 2.76 & 1.41 & 1.32 & 1.29 & 1.25 & 1.23 & 1.22 \\
\hline 7355900 & 1.03 & 1.24 & 1.39 & 1.58 & 1.72 & 1.86 & 1.51 & 1.43 & 1.39 & 1.34 & 1.31 & 1.29 \\
\hline 7356000 & 12.33 & 16.04 & 18.25 & 20.84 & 22.60 & 24.22 & 1.95 & 1.92 & 1.90 & 1.88 & 1.86 & 1.84 \\
\hline 7356500 & 5.25 & 6.66 & 7.67 & 8.91 & 9.77 & 10.60 & 2.00 & 2.00 & 2.00 & 2.00 & 2.00 & 2.00 \\
\hline 7356700 & 2.91 & 3.74 & 4.24 & 4.87 & 5.32 & 5.77 & 1.63 & 1.52 & 1.47 & 1.41 & 1.38 & 1.34 \\
\hline 7357700 & 2.68 & 3.38 & 3.77 & 4.22 & 4.53 & 4.83 & 1.43 & 1.35 & 1.31 & 1.27 & 1.25 & 1.23 \\
\hline 7359500 & 13.80 & 18.22 & 20.78 & 23.51 & 25.42 & 27.12 & 1.99 & 1.98 & 1.98 & 1.97 & 1.97 & 1.97 \\
\hline 7359520 & 2.01 & 2.14 & 2.32 & 2.65 & 2.99 & 3.40 & 1.68 & 1.42 & 1.32 & 1.24 & 1.20 & 1.16 \\
\hline 7359700 & 12.58 & 16.27 & 18.25 & 20.35 & 21.71 & 22.92 & 1.98 & 1.93 & 1.89 & 1.85 & 1.82 & 1.79 \\
\hline 7359750 & 2.92 & 3.78 & 4.44 & 5.24 & 5.80 & 6.33 & 1.43 & 1.30 & 1.25 & 1.21 & 1.18 & 1.17 \\
\hline 7359800 & 12.70 & 15.75 & 17.42 & 19.23 & 20.42 & 21.51 & 1.97 & 1.96 & 1.95 & 1.94 & 1.93 & 1.92 \\
\hline 7360150 & 0.78 & 1.23 & 1.58 & 2.09 & 2.51 & 2.96 & 2.00 & 2.00 & 2.00 & 2.00 & 2.00 & 2.00 \\
\hline 7360800 & 11.71 & 15.05 & 17.09 & 19.48 & 21.14 & 22.73 & 1.90 & 1.86 & 1.84 & 1.82 & 1.80 & 1.78 \\
\hline 7361020 & 1.15 & 1.55 & 1.82 & 2.16 & 2.41 & 2.66 & 1.40 & 1.26 & 1.21 & 1.16 & 1.14 & 1.12 \\
\hline 7361180 & 6.57 & 6.90 & 6.82 & 6.83 & 6.84 & 6.86 & 1.62 & 1.54 & 1.51 & 1.47 & 1.45 & 1.42 \\
\hline 7361200 & 8.11 & 8.84 & 9.14 & 9.46 & 9.70 & 10.00 & 1.92 & 1.77 & 1.69 & 1.61 & 1.55 & 1.51 \\
\hline 7361500 & 11.25 & 13.90 & 15.43 & 17.17 & 18.23 & 19.18 & 1.89 & 1.86 & 1.85 & 1.83 & 1.84 & 1.83 \\
\hline 7361680 & 1.68 & 2.16 & 2.45 & 2.80 & 3.05 & 3.29 & 1.27 & 1.21 & 1.18 & 1.15 & 1.14 & 1.12 \\
\hline $736 ! 780$ & 3.57 & 3.88 & 3.94 & 3.96 & 3.97 & 4.00 & 1.98 & 1.88 & 1.81 & 1.71 & 1.65 & 1.60 \\
\hline 7361800 & 9.96 & 10.46 & 10.70 & 10.95 & 11.12 & 11.27 & 1.84 & 1.74 & 1.69 & 1.64 & 1.61 & 1.58 \\
\hline 7362050 & 2.84 & 2.83 & 2.91 & 3.21 & 3.54 & 3.88 & 1.90 & 1.59 & 1.46 & 1.35 & 1.31 & 1.27 \\
\hline 7362100 & 7.55 & 9.45 & 9.91 & 10.27 & 10.50 & 10.75 & 2.00 & 1.88 & 1.78 & 1.67 & 1.60 & 1.55 \\
\hline 7362330 & 2.72 & 3.41 & 4.05 & 4.74 & 5.16 & 5.59 & 1.62 & 1.41 & 1.34 & 1.29 & 1.26 & 1.24 \\
\hline 7362450 & 3.42 & 3.60 & 3.78 & 4.02 & 4.21 & 4.40 & 1.80 & 1.58 & 1.49 & 1.41 & 1.37 & 1.33 \\
\hline 7362500 & 6.95 & 7.82 & 8.00 & 8.18 & 8.32 & 8.46 & 1.97 & 1.79 & 1.68 & 1.59 & 1.54 & 1.49 \\
\hline 7363000 & 14.38 & 18.78 & 20.73 & 22.32 & 23.13 & 23.75 & 1.99 & 1.98 & 1.96 & 1.91 & 1.88 & 1.84 \\
\hline 7363050 & 1.50 & 2.09 & 2.50 & 3.05 & 3.48 & 3.92 & 1.24 & 1.20 & 1.18 & 1.16 & 1.14 & 1.13 \\
\hline 7363200 & 11.98 & 12.45 & 12.64 & 12.86 & 13.04 & 13.29 & 1.82 & 1.68 & 1.61 & 1.54 & 1.50 & 1.47 \\
\hline 7363300 & 5.58 & 6.48 & 7.14 & 8.10 & 8.81 & 9.49 & 1.51 & 1.39 & 1.33 & 1.28 & 1.26 & 1.24 \\
\hline 7363330 & 2.15 & 2.62 & 2.93 & 3.34 & 3.64 & 3.93 & 1.47 & 1.33 & 1.28 & 1.23 & 1.21 & 1.19 \\
\hline 7363430 & 1.30 & 1.60 & 1.89 & 2.36 & 2.72 & 3.09 & 1.49 & 1.30 & 1.23 & 1.17 & 1.15 & 1.13 \\
\hline 7363450 & 1.21 & 1.66 & 1.95 & 2.29 & 2.53 & 2.76 & 1.42 & 1.30 & 1.26 & 1.21 & 1.19 & 1.17 \\
\hline 7363500 & 13.99 & 14.77 & 14.99 & 15.33 & 15.61 & 15.91 & 1.99 & 1.81 & 1.72 & 1.64 & 1.59 & 1.55 \\
\hline 7364030 & 0.74 & 0.97 & 1.09 & 1.24 & 1.34 & 1.43 & 1.32 & 1.24 & 1.21 & 1.19 & 1.17 & 1.16 \\
\hline 7364070 & 2.68 & 2.87 & 2.99 & 3.13 & 3.23 & 3.34 & 1.80 & 1.66 & 1.60 & 1.54 & 1.50 & 1.47 \\
\hline 7364260 & 2.22 & 2.74 & 3.03 & 3.28 & 3.44 & 3.59 & 1.39 & 1.33 & 1.30 & 1.28 & 1.27 & 1.25 \\
\hline 7364300 & 6.80 & 8.17 & 8.48 & 8.90 & 9.29 & 9.70 & 1.99 & 1.82 & 1.71 & 1.60 & 1.54 & 1.50 \\
\hline 7364550 & 0.86 & 1.08 & 1.23 & 1.42 & 1.57 & 1.72 & 1.12 & 1.09 & 1.08 & 1.07 & 1.06 & 1.05 \\
\hline 7364700 & 3.66 & 5.46 & 6.88 & 8.60 & 9.81 & 10.98 & 2.00 & 2.00 & 1.98 & 1.93 & 1.89 & 1.84 \\
\hline 7365800 & 4.76 & 5.15 & 5.81 & 7.05 & 8.15 & 9.35 & 1.63 & 1.40 & 1.31 & 1.24 & 1.21 & 1.18 \\
\hline 7365900 & 4.00 & 5.53 & 6.14 & 6.74 & 7.16 & 7.61 & 2.00 & 1.90 & 1.80 & 1.68 & 1.61 & 1.54 \\
\hline 7366000 & 7.51 & 9.03 & 8.90 & 9.06 & 9.41 & 9.88 & 2.00 & 1.88 & 1.74 & 1.61 & 1.53 & 1.48 \\
\hline 7366200 & 4.14 & 5.49 & 6.01 & 6.73 & 7.34 & 8.00 & 1.99 & 1.88 & 1.79 & 1.69 & 1.64 & 1.60 \\
\hline
\end{tabular}


where,

Q, A, S, L, P, E are as previously defined,

$\mathrm{R}=$ mean weighted hydraulic radius. Defined as the summation of the hydraulic radius times the discharge in each subsection divided by the total discharge.

$\mathrm{N}=$ discharge in the main channel times 2 plus the flood plain discharge, divided by the total discharge. $N$ ranges from 1 to 2 . $N=1$ if all water is on flood plain. $\mathrm{N}=2$ if all water is in main channel.

When most of the discharge is in the main channe1, the equations tend to overestimate discharge. For this reason, $N$ was used in the equations to compensate for the overestimation of discharge. The standard error of the equations was reduced about 4 percent when $\mathrm{N}$ was included.

The standard error of equations 17-28 using this procedure is about 9 percent less than the standard error of equations 2-13. The user must decide if this increase in accuracy is worth the effort of estimating a cross section and stage-discharge relation.

\section{Estimating the Cross Section}

The cross section of the channel and flood plain can be determined using the contour lines on a topographic map. The position of the cross section at the ungaged site is drawn on the map normal to flow. Care should be taken to assure that it is a typical valley cross section. The station (lateral distance from an arbitrary point above high water) where these contours cross the cross section is tabulated and plotted. Additional points for determining the cross section can be transferred from other points, either upstream or downstream to the site by adjusting for the slope and distance. All points in the cross section are important but particular care must be taken in determining elevation of bankful stage, elevation of the bottom of the channel and channel width.

The contour lines that cross the channel represent low water at the time the photographs for mapping were taken. The depth of water during low water conditions should be estimated.

The elevation of the bottom of the channel is determined by interpolating between points where the contours cross the channel and subtracting the depth from this value. The elevation of bankful stage can be determined along the channel where the contour line breaks out of the channel and begins to go into the flood plain. This point can be transferred to the site by adjusting it for slope and distance using slope of the flood plain from contour crossings of the channel and distance from the cross section. The channel width can be estimated by measuring this width at several points near the site and using an average or typical value. The entire cross section can be determined in the field by transit-stadia survey. In determining hydraulic radius for analysis in this report all cross sections were determined from topographic maps using the procedure described above. 
An example for determining the cross section from topographic maps is described. A typical cross section is drawn on the topographic map as shown on figure 5. This section does not need to be exactly at the site. It can be moved either upstream or downstream to where the cross section appears typical of the reach. The station (lateral distance) where the contour lines cross the cross section is determined and plotted on figure 5. In this example all points are measured from where the $350-\mathrm{ft}$ contour crosses the cross section.

The bottom of the channel is determined by interpolating between where adjacent contour lines cross the channel. The flood plain distance between where the contour lines cross the channel is $770 \mathrm{ft}$. The contour interval is $10 \mathrm{ft}$. Therefore, the flood plain slope is 10 divided by 770 or 0.013 $\mathrm{ft} / \mathrm{ft}$. The cross section is $525 \mathrm{ft}$ upstream from where the $320-\mathrm{ft}$ contour line crosses the channel. Multiplying distance by slope $(525 \times 0.013=6.8)$, the bottom of the channel is $326.8 \mathrm{ft}(320+6.8)$ at the cross section. This point was actually the water-surface elevation when the photographs for mapping were made. If the approximate depth of low water is known, it can be subtracted from this point. The distance along the main channel between the contour lines is $850 \mathrm{ft}$. Therefore, the main channel slope is 10 divided by 850 or $0.0118 \mathrm{ft} / \mathrm{ft}$.

Bankful stage is where the 330-ft contour breaks out of the channel and goes onto the flood plain. This is about $170 \mathrm{ft}$ downstream from the cross section. Multiplying distance by slope $(170 \times 0.013=2.2)$, bankful stage at the cross section is $332.2 \mathrm{ft}(330+2.2)$. The elevation of bankful stage and channel bottom is plotted on figure 5 and the cross section is drawn by connecting points. Judgements are made when connecting points either by a straight line or a curved line.

\section{Stage-Discharge Relation} method.

Stage-discharge relations are usually determined by the slope-conveyance

$$
Q=\frac{1.486}{n} A_{1}\left(R^{0.67}\right) s_{f}^{0.50}
$$

where,

$$
\begin{aligned}
\mathrm{Q} & =\text { discharge in } \mathrm{ft}^{3} / \mathrm{s}, \\
\mathrm{n} & =\text { Manning's roughnes coefficient, } \\
\mathrm{A}_{1} & =\text { cross sectional area of stream in } \mathrm{ft}^{2}, \\
\mathrm{R} & =\text { hydraulic radius in } \mathrm{ft} \text {, defined as area divided by the wetted } \\
& \text { perimeter, and } \\
\mathrm{S}_{\mathrm{f}} & =\text { slope of the energy gradient in } \mathrm{ft} / \mathrm{ft} .
\end{aligned}
$$


5A
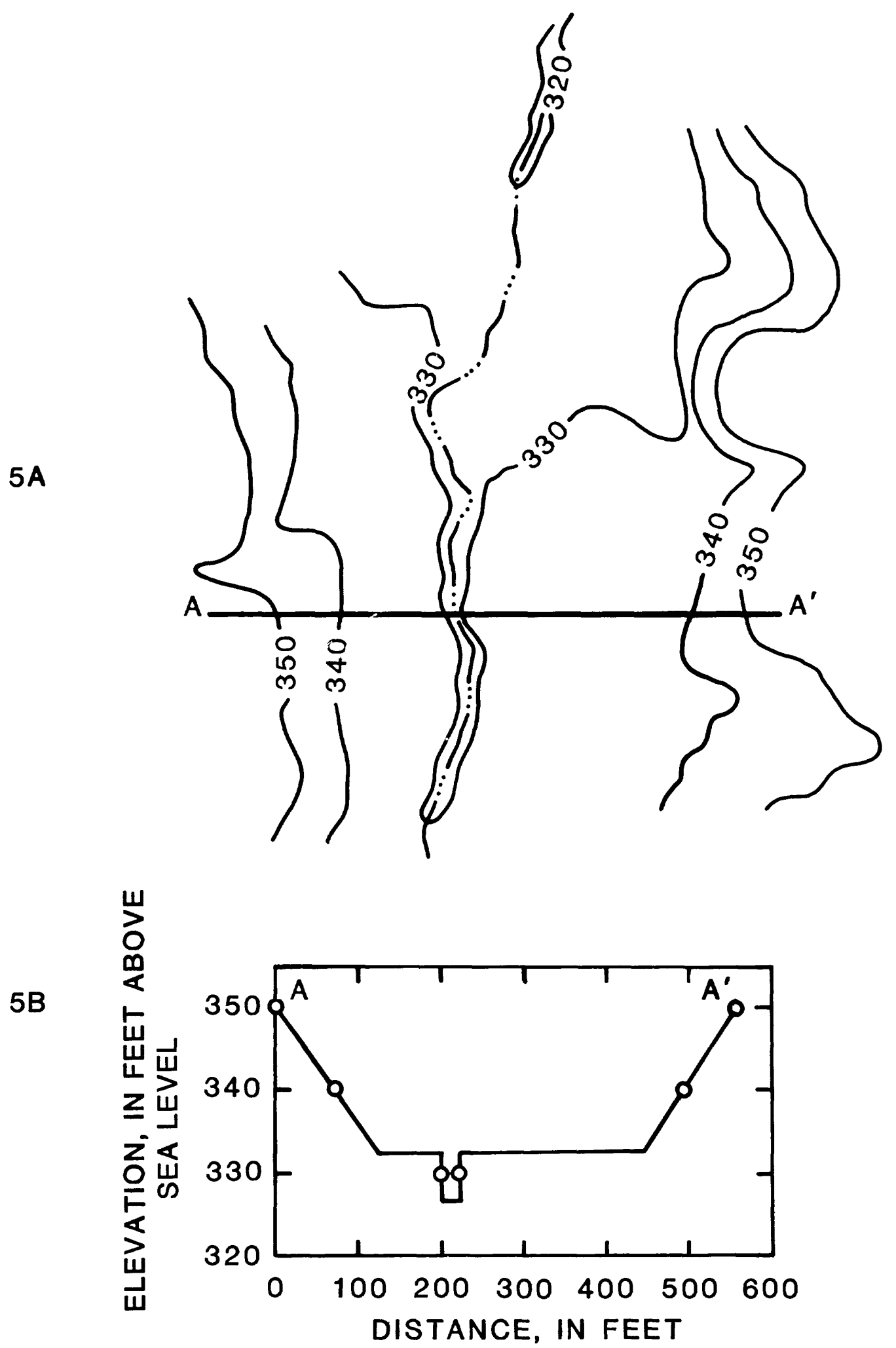

Figure 5.--Cross section of valley from topographic map. 
The slope is determined for both the main channel and for the flood plain. The elevations used in determining the slope are picked from the topographic map where the contours cross the main channel. The distance between these points is measured along the main channel when determining the main channel slope and measured along a line parallel to the flood plain flow when determining the flood plain slope. The cross section of the channel and flood plain is used in determining the discharge. The cross section is subdivided so that a discharge can be determined for the main channel and for the flood plain. The cross section on the flood plain is further subdivided if there is a major change in shape.

Manning's roughness coefficient is selected in the field and is difficult at times to determine. Engineering judgement must be used in making the selection. Manning's roughness coefficient, $n$, which is needed in equation 29 to compute discharge is determined based on the work done by Jarrett (1985) and Riggs (1976). Both Jarrett and Riggs showed that, $\mathrm{n}$, can be related to slope and to elements of the cross section. Jarrett derived the equation

where,

$$
\mathrm{n}=0.39 \mathrm{~s}_{\mathrm{f}}^{0.38} \mathrm{R}^{-0.16}
$$

$\mathrm{n}=$ Manning's coefficient

$S_{f}=$ slope of the energy line in $\mathrm{ft} / \mathrm{ft}$, and

$\mathrm{R}=$ hydraulic radius in $\mathrm{ft}$.

This equation is based on data collected for 75 storm events at 22 stations in Colorado. These are high gradient streams with an average slope of about $62 \mathrm{ft} / \mathrm{mi}$. This equation can be substituted in equation 29 which would eliminate " $n$ " and form the equation

$$
Q=3.81 A_{1} R^{0.83} \mathrm{~S}_{\mathrm{f}}{ }^{0.12}
$$

Riggs derived the equation

where,

$$
\mathrm{Q}=3.39 \mathrm{~A}_{1}^{1.30} \mathrm{~s}^{0.32}
$$

$Q=$ discharge in $\mathrm{ft}^{3} / \mathrm{s}$,

$\mathrm{A}_{1}=$ cross sectional area in $\mathrm{ft}^{2}$, and

$S=$ slope of water surface in $\mathrm{ft} / \mathrm{ft}$.

This equation is based on data collected at 30 stations throughout the United States. These are low gradient streams with a slope that ranges from 2 to $95 \mathrm{ft} / \mathrm{mi}$ with an average of 20 .

Both Jarrett and Riggs simplified the computation of discharge by not using Manning's " $n$ " in their equations. 
The slope of the 200 Arkansas streams used in the study ranges from 0.41 to $609 \mathrm{ft} / \mathrm{mi}$ with a median of 17.5. Both Jarrett's and Riggs' equations which apply to the main channel only are believed to be applicable to Arkansas streams. The slope at 123 stations is applicable to Jarrett's equation for high gradient streams. The slope at 165 stations is applicable to Rigg's equation for low gradient streams. Eighty-eight stations overlap and are applicable to both equations. Rather than use Jarrett's equation for high gradient streams and Rigg's equation for low gradient streams, an attempt was made to combine the data used in deriving both equations and derive an equation that can be used for all streams. Riggs did not use the hydraulic radius in his equation although these data were available for most of his stations. At stations where the hydraulic radius was not available, these data were estimated based on a relation between area and hydraulic radius developed using the other stations. The slope of the water surface, s, and the slope of the energy gradient, $S_{f}$, is assumed to be parallel. Data used for both Jarrett's and Riggs' equations were combined and used in a regression analysis to form a single relation for the main channel as shown below:

$$
Q=7.13 \mathrm{~A}_{1}^{0.94} \mathrm{R}^{0.80} \mathrm{~S}^{0.20}
$$

An equation for computing discharge on the flood plain was developed so that a theoretical rating curve could be developed at each site that would closely parallel the actual rating curve. The theoretical rating curve is determined by adding the discharge computed for the main channel to the discharge computed for the flood plain. The equation for computing discharge on the flood plain was developed by assuming that the exponents on $A, R$, and $S$ in equation 33 would remain the same and only the constant would change. The constant in the equation was determined for each site so that the difference between the 100-year stage and the 2-year stage was the same for both the theoretical rating curve and the actual rating curve. The median value of these constants was used in the final equation. The equation for computing discharge on the flood plain is

$$
Q=1.30 \mathrm{~A}_{1}^{0.94} \mathrm{R}^{0.80} \mathrm{~S}^{0.20}
$$

The difference in the constants in equation 33 and 34 means that the " $n$ " value on the flood plain is about five times greater than the " $n$ " in the main channel. Note that the slopes used in equations 33 and 34 are for the main channel and for the flood plain, respectively.

At several selected stages, the discharge is computed for the main channel and for the flood plain. These discharges are added to form the total discharge for that stage. The stage-discharge relation is made by plotting stage against discharge. This relation needs to be developed between the discharges of the 2-year and 100-year floods. 
An example for determining the stage-discharge relation, the hydraulic radius, and $\mathrm{N}$ is described. The cross section for the site is shown on figure 5. The cross-section properties and discharge are computed for the left flood plain (looking downstream), the main channel, and the right flood plain. The area, wetted perimeter, hydraulic radius, and discharge are computed for each subsection at selected stages. The computations are shown in table 6. Below each selected stage, the area is computed for each subsection. The wetted perimeter is the distance along the ground for each subsection. The wetted perimeter is always equal to or greater than the width. The hydraulic radius is the area divided by the wetted perimeter.

At each selected stage, the discharge is computed for the main channel using equation 33 and for each flood plain using equation 34 . The slope of the main channel is used in equation 33 and the slope of the flood plain is used in equation 34. The total discharge for each stage is the summation of the discharges for each subsection. The stage-discharge relation is shown on figure 6.

\section{Discharge-Frequency Curve}

An example of computing the discharge-frequency curve for the site shown on figure 5 is described. The basin and climatic characteristics for this site (in Region B) are:

\footnotetext{
Drainage area $=4.0 \mathrm{mi}^{2}$,

Mean annual precipitation $=50.0$ inches,

Slope of main channel $=90.0 \mathrm{ft} / \mathrm{mi}$,

Mean basin elevation $=1,490 \mathrm{ft}$,

Slope of flood plain $=0.0130 \mathrm{ft} / \mathrm{ft}$, and

Slope of main channel $=0.0119 \mathrm{ft} / \mathrm{ft}$.
}

The preliminary discharges are computed using equation 8-13. The computed discharges are shown below.

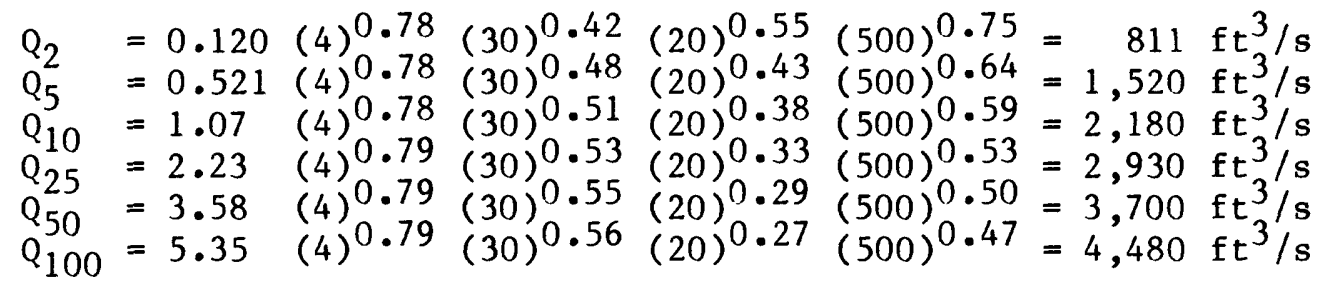

The mean weighted hydraulic radius and $\mathrm{N}$ are needed in equations 23-28 for computing the discharge for each recurrence interval. The mean weighted hydraulic radius shown in table 6 is computed as the summation of the hydraulic radius times the discharge in each subsection divided by the total discharge. The mean weighted hydraulic radius is plotted against discharge on figure 6 .

The $\mathrm{N}$ shown in table 6 is computed as the discharge in the main channel times 2 plus the flood plain discharge, divided by the total discharge. The value of $\mathrm{N}$ is plotted against discharge on figure 6 . 
Table 6.--Computation of discharge and cross-sectional properties

\begin{tabular}{|c|c|c|c|c|c|c|c|c|c|c|c|c|c|c|c|}
\hline \multicolumn{4}{|c|}{ Left flood plain } & \multicolumn{5}{|c|}{ Main channel } & \multicolumn{3}{|c|}{ Right flood pla1n } & \multirow[b]{2}{*}{$\begin{array}{l}\text { Dis- } \\
\text { charge } \\
\text { (cub1c } \\
\text { feet } \\
\text { per } \\
\text { second) } \\
\end{array}$} & \multirow[b]{2}{*}{$\begin{array}{l}\text { Total } \\
\text { dis- } \\
\text { charge } \\
\text { (cubic } \\
\text { feet per } \\
\text { second) }\end{array}$} & \multirow[b]{2}{*}{$\begin{array}{l}\text { Mean } \\
\text { welghted } \\
\text { hydraulic } \\
\text { radius } \\
\text { (feet) }\end{array}$} & \multirow[b]{2}{*}{$\mathbf{N}$} \\
\hline $\begin{array}{r}\text { Stage } \\
\text { (feet) }\end{array}$ & $\begin{array}{c}\text { Area } \\
\text { (square } \\
\text { feet) }\end{array}$ & $\begin{array}{l}\text { Wetted } \\
\text { peri- } \\
\text { meter } \\
\text { (feet) }\end{array}$ & $\begin{array}{c}\text { Hydrau- } \\
\text { Ilc } \\
\text { radius } \\
\text { (feet) }\end{array}$ & $\begin{array}{l}\text { Dis- } \\
\text { charge } \\
\text { (cubic } \\
\text { feet } \\
\text { per } \\
\text { second) } \\
\end{array}$ & $\begin{array}{c}\text { Area } \\
\text { (square } \\
\text { feet) }\end{array}$ & $\begin{array}{l}\text { Wetted } \\
\text { peri- } \\
\text { meter } \\
\text { (feet) }\end{array}$ & $\begin{array}{c}\text { Hydrau- } \\
\text { lic } \\
\text { radius } \\
\text { (feet) }\end{array}$ & $\begin{array}{l}\text { Dis- } \\
\text { charge } \\
\text { (cublc } \\
\text { feet } \\
\text { per } \\
\text { second) }\end{array}$ & $\begin{array}{c}\text { Area } \\
\text { (square } \\
\text { feet) }\end{array}$ & $\begin{array}{l}\text { Wetted } \\
\text { pert- } \\
\text { meter } \\
\text { (feet) }\end{array}$ & $\begin{array}{l}\text { Hydrau- } \\
\text { lic } \\
\text { radius } \\
\text { (feet) }\end{array}$ & & & & \\
\hline $\begin{array}{l}331 \\
332.2 \\
333 \\
334 \\
335 \\
336 \\
337 \\
338\end{array}$ & $\begin{array}{c}0 \\
58.3 \\
137.8 \\
224.6 \\
318.7 \\
420.1 \\
528.8\end{array}$ & $\begin{array}{r}70 \\
75.9 \\
83.3 \\
90.6 \\
98.0 \\
105.4 \\
112.8\end{array}$ & $\begin{array}{l}0 \\
0.77 \\
1.65 \\
2.48 \\
3.25 \\
3.99 \\
4.69\end{array}$ & $\begin{array}{r}0 \\
20 \\
83 \\
183 \\
316 \\
482 \\
682\end{array}$ & $\begin{array}{r}92.4 \\
118.8 \\
136.4 \\
158.4 \\
180.4 \\
202.4 \\
224.4 \\
246.4\end{array}$ & $\begin{array}{l}30.4 \\
32.8 \\
32.8 \\
32.8 \\
32.8 \\
32.8 \\
32.8 \\
32.8\end{array}$ & $\begin{array}{l}3.04 \\
3.62 \\
4.16 \\
4.83 \\
5.50 \\
6.17 \\
6.84 \\
7.51\end{array}$ & $\begin{array}{r}503 \\
732 \\
932 \\
1,209 \\
1,516 \\
1,852 \\
2,215 \\
2,607\end{array}$ & $\begin{array}{c}0 \\
178.1 \\
406.7 \\
642.0 \\
883.9 \\
1,132 \\
1,388\end{array}$ & $\begin{array}{l}220 \\
225.4 \\
232.1 \\
238.8 \\
245.5 \\
252.2 \\
258.9\end{array}$ & $\begin{array}{l}0 \\
0.79 \\
1.75 \\
2.69 \\
3.60 \\
4.49 \\
5.36\end{array}$ & $\begin{array}{r}0 \\
59 \\
242 \\
524 \\
894 \\
1,346 \\
1,879\end{array}$ & $\begin{array}{r}503 \\
732 \\
1,011 \\
1,534 \\
2,223 \\
3,062 \\
4,043 \\
5,168\end{array}$ & $\begin{array}{l}3.04 \\
3.62 \\
3.89 \\
4.17 \\
4.59 \\
5.12 \\
5.72 \\
6.36\end{array}$ & $\begin{array}{l}2.00 \\
2.00 \\
1.92 \\
1.79 \\
1.68 \\
1.60 \\
1.55 \\
1.50\end{array}$ \\
\hline
\end{tabular}




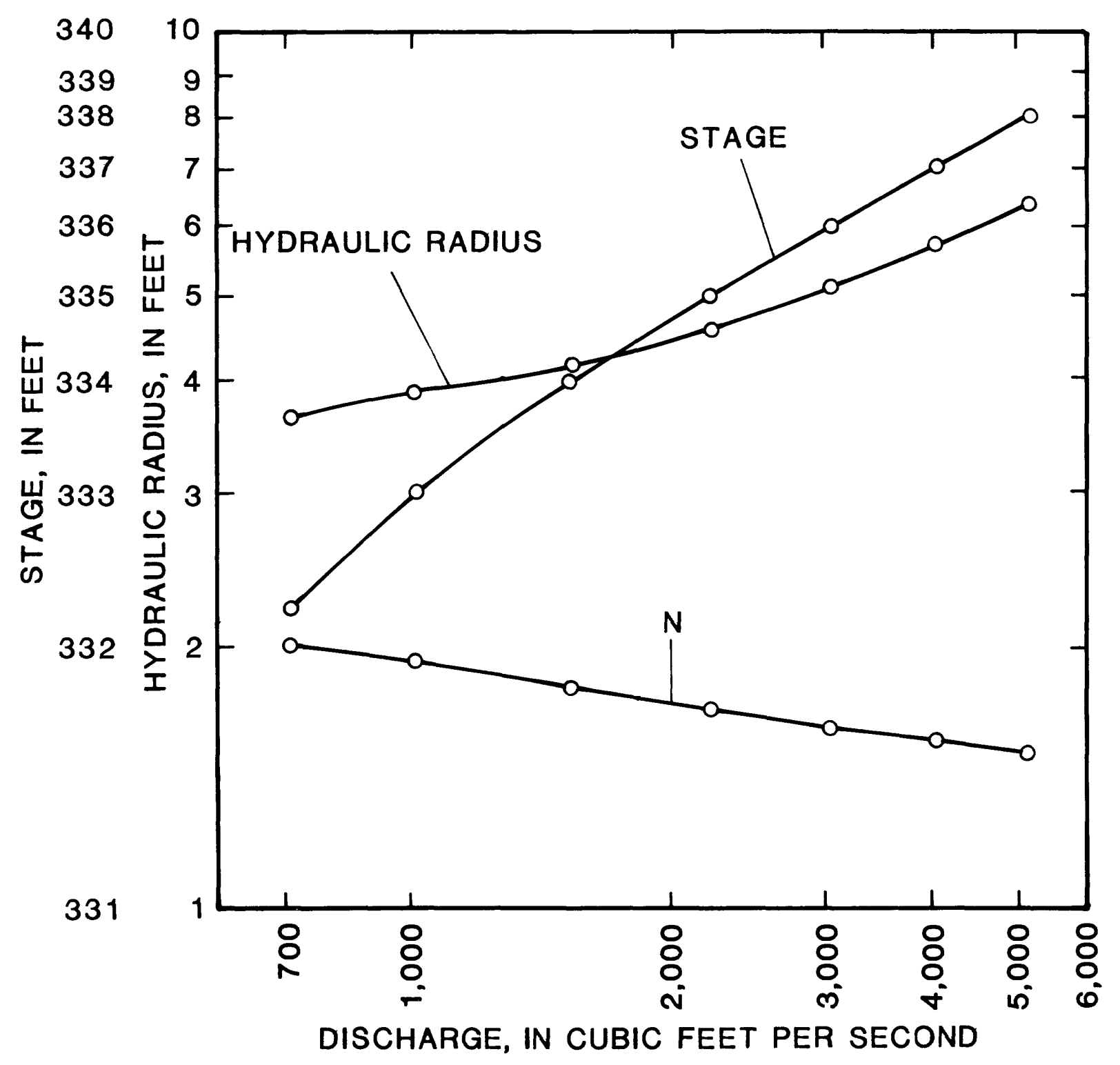

Figure 6.--Stage-discharge, hydraulic radius-discharge, and $\mathrm{N}$-discharge relation. 
The preliminary discharge for each recurrence interval computed using equations 8-13 are entered in figure 6 to determine the mean weighted hydraulic radius and $\mathrm{N}$. The mean weighted hydrualic radius and $\mathrm{N}$ for each recurrence interval are shown below.

\begin{tabular}{lccc}
$\begin{array}{l}\text { Recurrence } \\
\text { interval, } \\
\text { in years }\end{array}$ & $\begin{array}{l}\text { Preliminary } \\
\text { discharge, } \\
\text { cubic feet } \\
\text { per second }\end{array}$ & $\begin{array}{c}\text { Mean weighted } \\
\text { hydraulic radius, } \\
\text { in feet }\end{array}$ & $\mathrm{N}$ \\
\hline & & & \\
2 & 811 & 4.72 & 1.97 \\
5 & 1,520 & 4.55 & 1.79 \\
10 & 2,180 & 5.05 & 1.69 \\
50 & 2,930 & 5.54 & 1.61 \\
100 & 3,700 & 5.99 & 1.57 \\
\hline
\end{tabular}

The discharge using the alternate procedure is computed using equations 23-28 and are shown below.

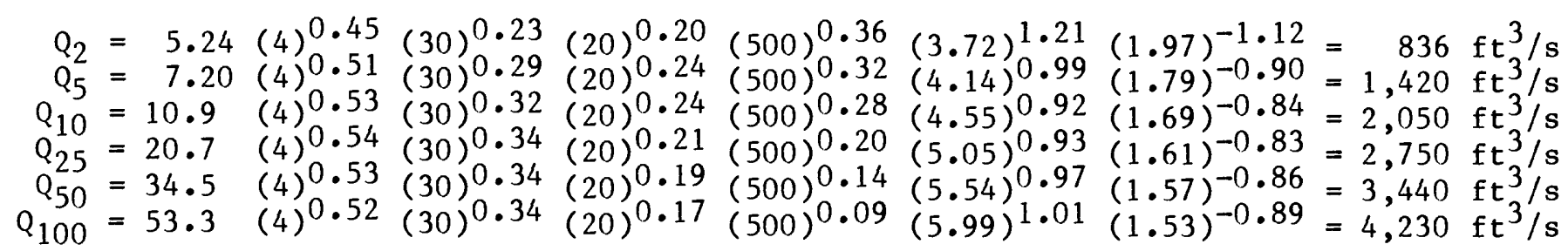

The values of the mean weighted hydraulic radius and $\mathrm{N}$ are not recomputed based on the results of equations $23-28$.

The computed discharges using the alternate equations differ slightly from the discharges computed using equations 8-13. The maximum difference is 7 percent. There is no way to know which set of equations give the best answer for any particular site. The average standard error of equations 23-28 is about 9 percent less than the standard error of equations 8-13.

FLOOD MAGNITUDE AND FREQUENCY ON LARGE RIVERS

Large rivers have floodflow characteristics differing from those of smaller tributary streams and were treated individually. The peak discharges for recurrence intervals of $2,5,10,25,50$, and 100 years for all gaging stations on large streams and regulated streams are listed in table 3 . The peak discharge for regulated streams reflects the pattern of regulation. If the pattern of regulation changes, the discharges will change. 
The Mississippi River is one of the longest rivers in the world. Its drainage basin extends from New York to Montana and includes about 40 percent of the conterminous United States. A flood-frequency study of such a river is outside the scope of this report. Flood-frequency relations at three gaging stations are shown in table 3 and can be used as a guide to estimate discharges at nearby places along the river. Data for the three gaging stations are plotted on figure 7 .

\section{St. Francis River}

Regulation began April 1, 1941 by Wappapello Reservoir. The storage capacity of Wappape1lo Reservoir is 625,000 acre-ft. Downstream from the gaging station at Lake City (07040450) the flow separates into several channels. The flood-frequency curves for St. Francis River, shown in figure 8 , include the effect of regulation and is the combined discharge of all channe1s.

\section{Black River}

The regulation by Clearwater Reservoir (storage capacity 413,700 acre$\mathrm{ft}$ ) has only a slight effect on flood peaks of Black River. Data collected prior to regulation at the gaging station near Corning (07064000) was used in developing the Region A frequency curves. The flood-frequency curves for Black River are shown on figure 9.

\section{Arkansas River}

The flow of Arkansas River is regulated by many locks, dams, and reservoirs. The flood-frequency curves shown on figure 10 include the effect of regulation. The flood-frequency curves for station 07250550 which is based on 15 years of record were adjusted to the 45-year period at the two other stations.

\section{White River}

The flow of White River is regulated by Beaver Lake, Table Rock Lake, Bu11 Shoals Lake, Norfork Lake, and Greers Ferry Lake. Upstream regulation begins at Beaver Lake near Eureka Springs where the drainage area is 1,192 $\mathrm{mi}^{2}$. The frequency curves on figure 11 show the effects of regulation. Upstream from Beaver lake, the discharge frequency should be computed using the regression equations and the gaging station data shown in table 2. 


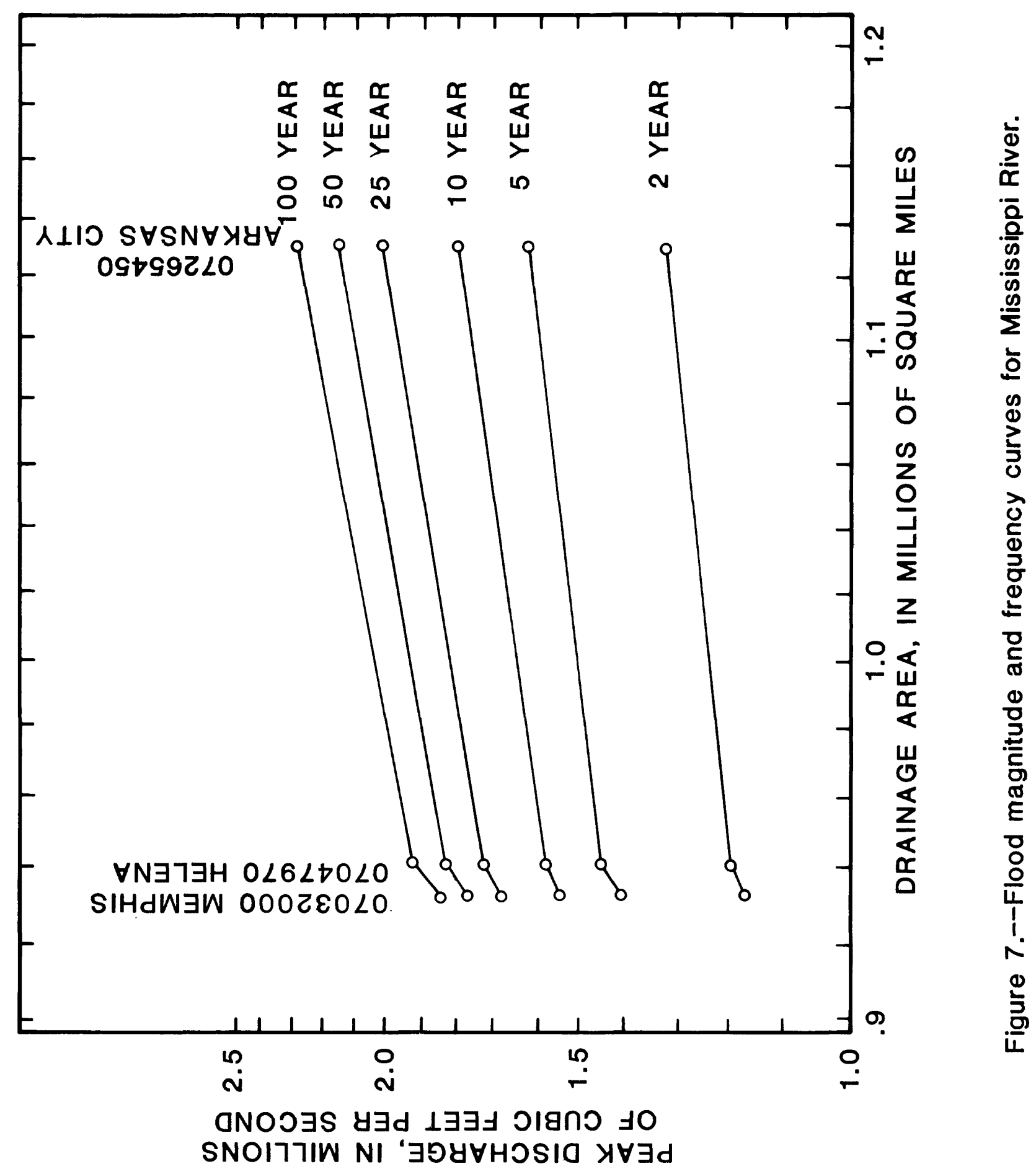




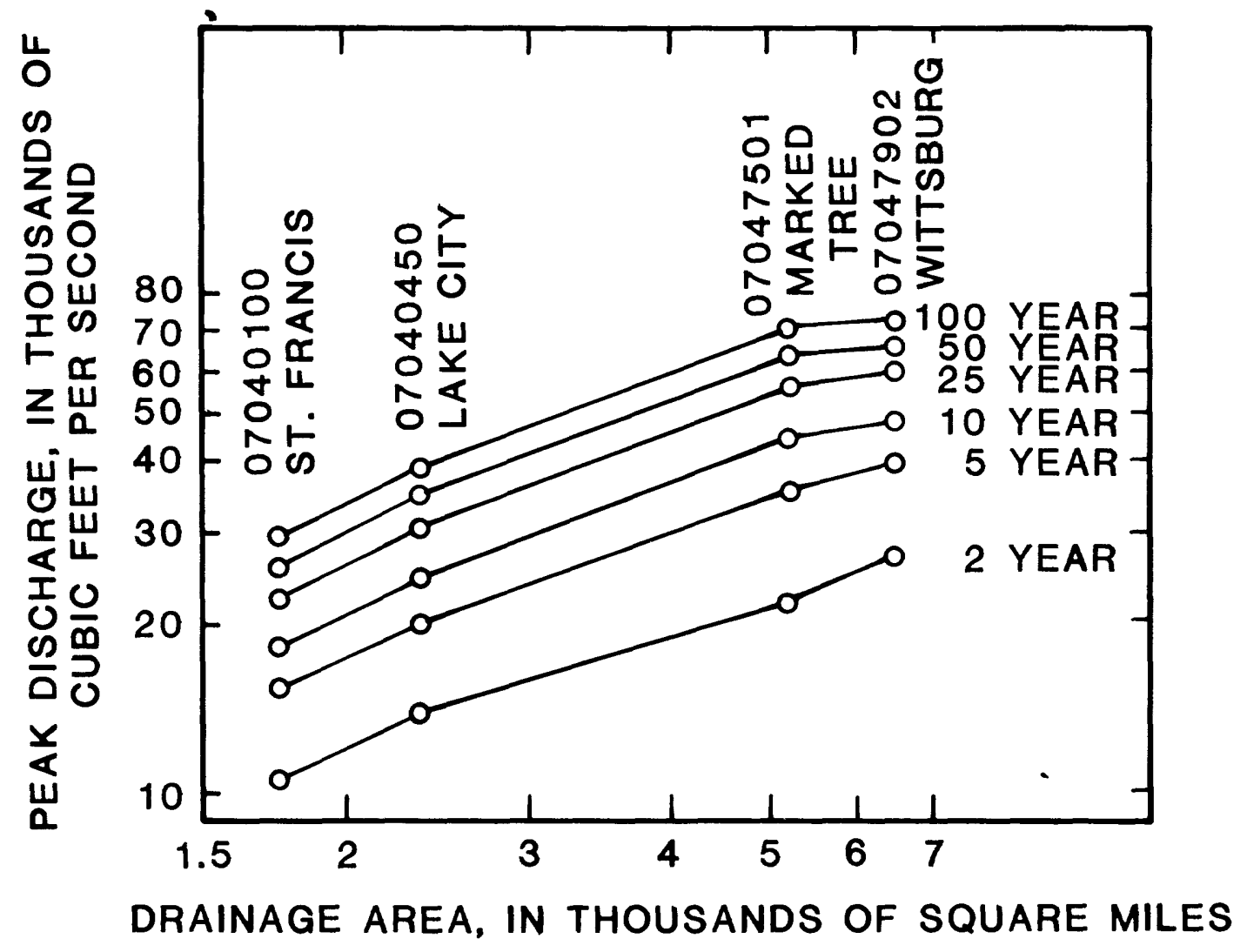

Figure 8.--Flood magnitude and frequency curves for St. Francis River. 


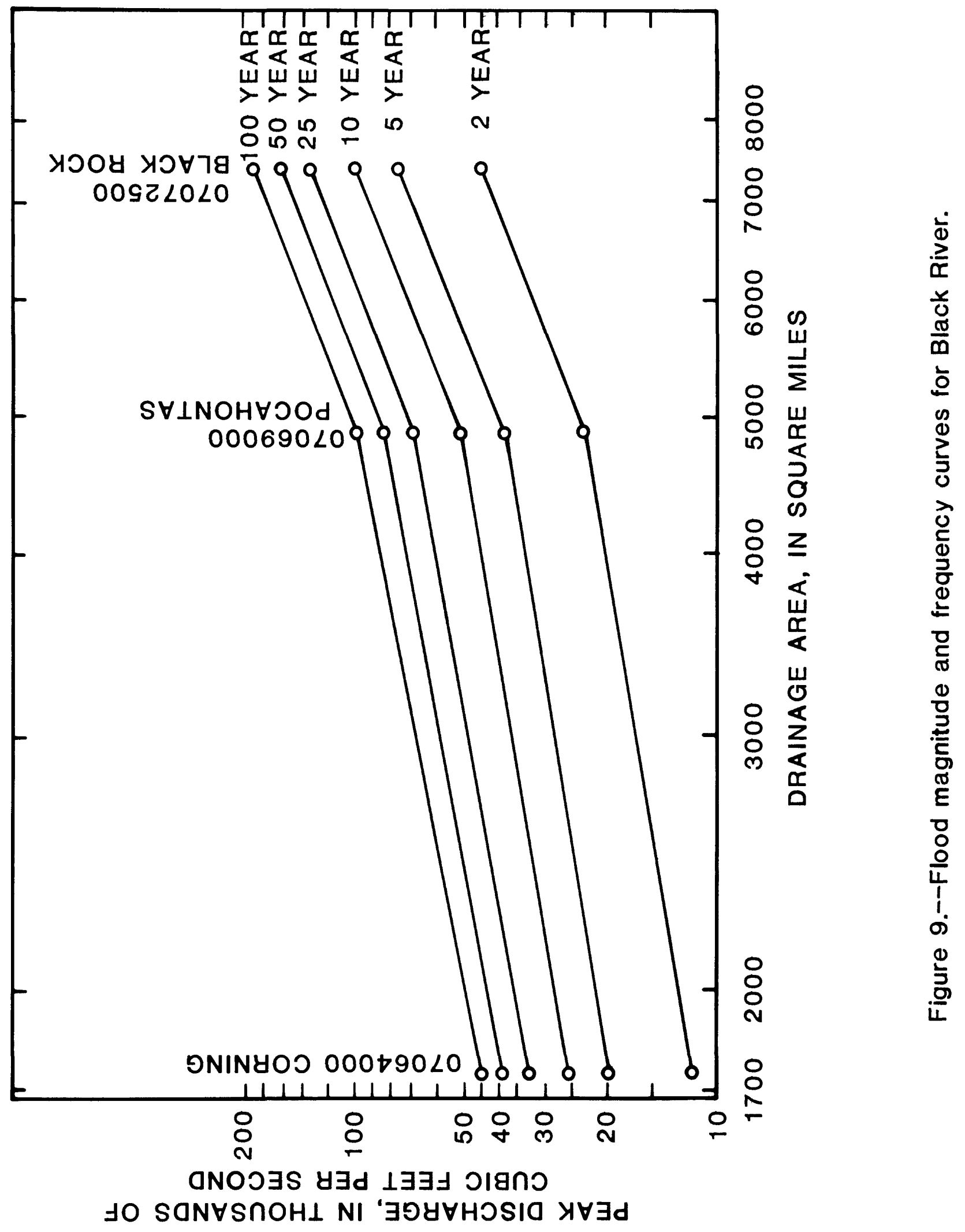




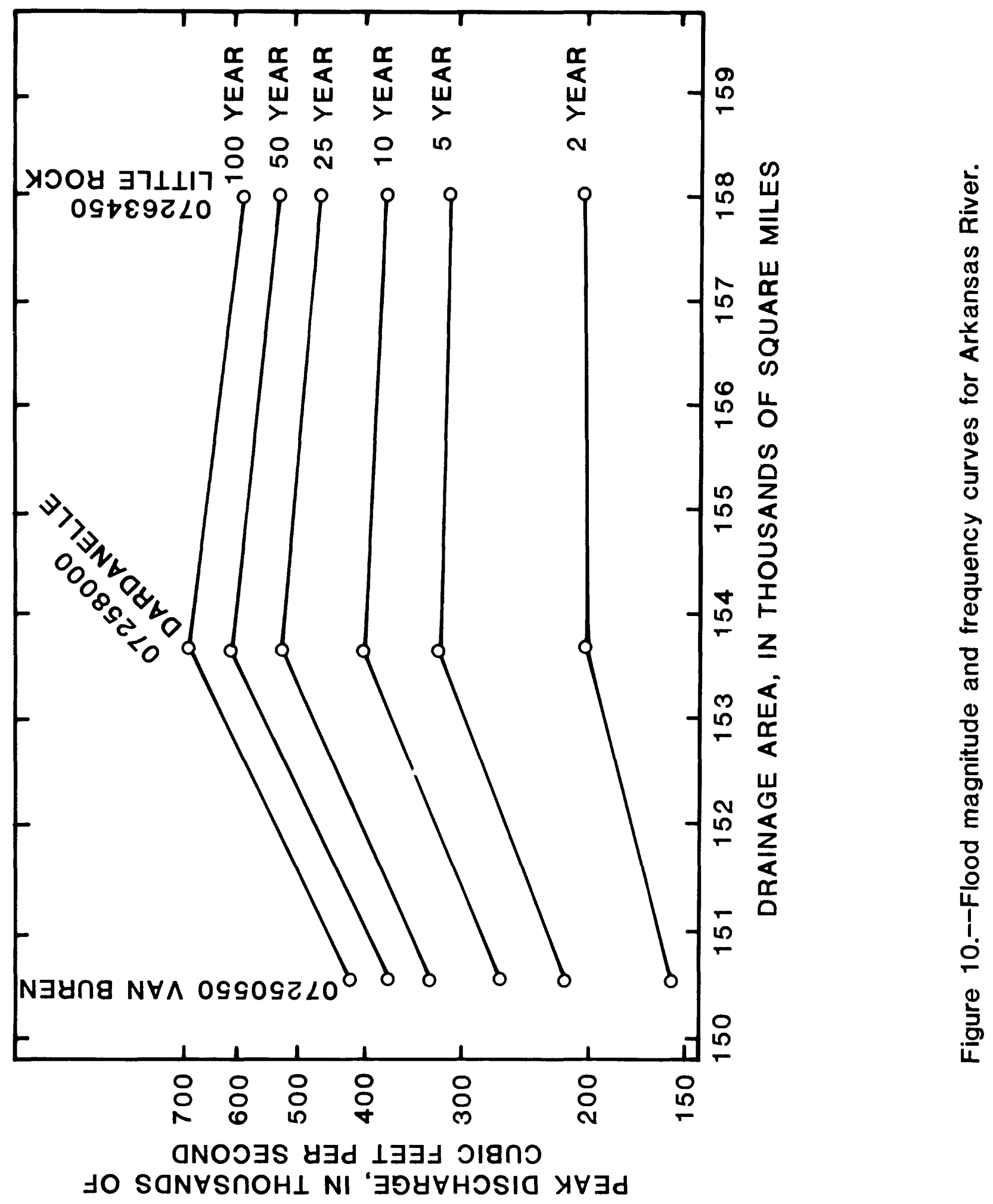




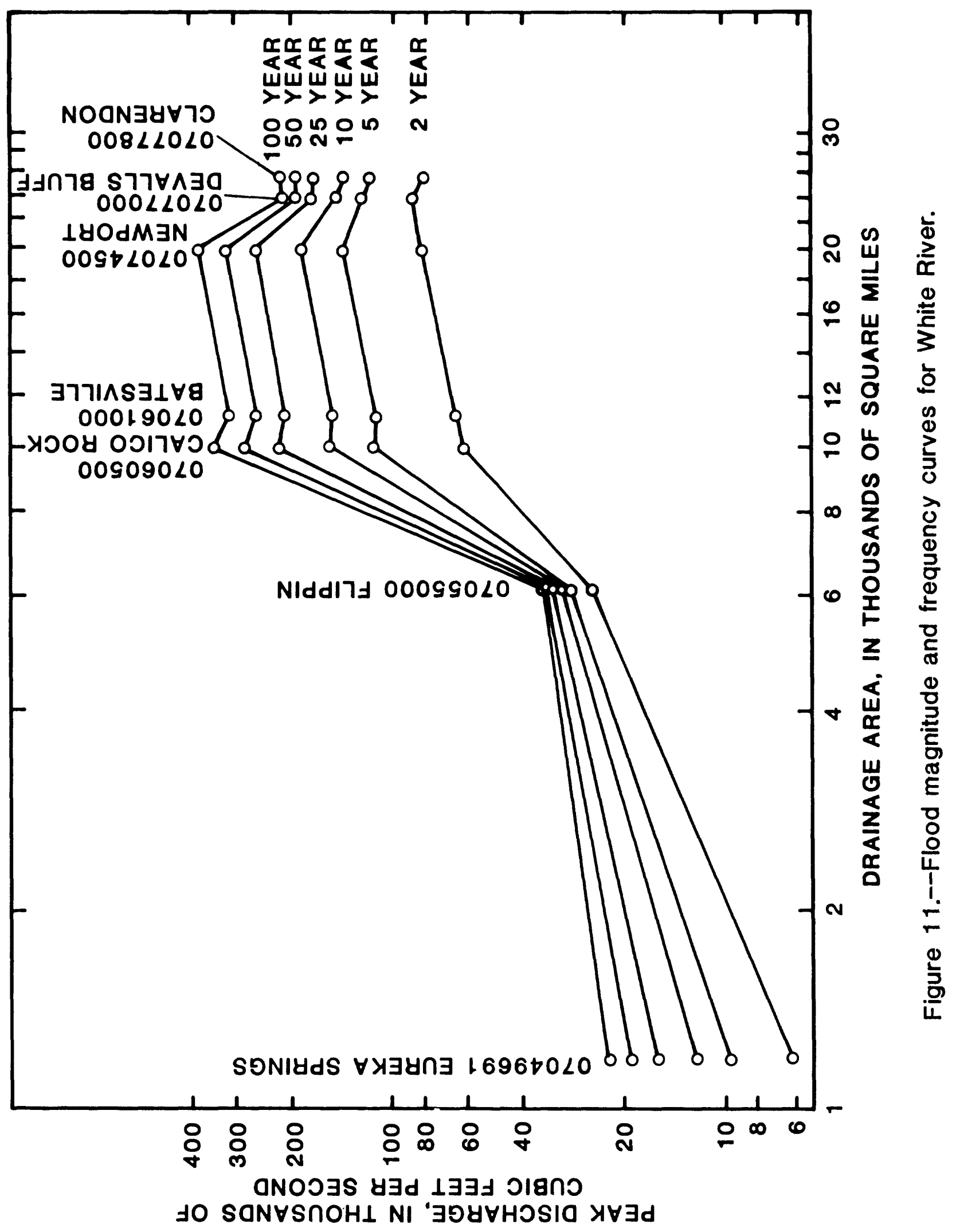




\section{Red River}

The flow of Red River is regulated by Lake Texoma, Pat Mayse Lake, Hugo Lake, Millwood Lake, and Wright Patman Lake. The flood-frequency curves on figure 12 include the effects of regulation.

\section{Ouachita River}

The flow of Duachita River is regulated by Lake Catherine, Lake Hamilton, and Lake Ouachita. Upstream regulation begins with Lake Ouachita at Blakely Mountain Dam which has a drainage area of $1,105 \mathrm{mi}^{2}$. The flood-frequency curves on figure 13 include the effects of regulation. Upstream from Lake Ouachita, the discharge frequency should be computed using the regression equations and the gaging station data shown in table 2 .

\section{FLOOD FREQUENCY FOR URBAN AREAS}

Very little data for urban areas in Arkansas are available. Data has been collected on three urban streams in Little Rock since 1980 and on three streams in Fort Smith since 1978. These data consist of peaks only and are not included in this report.

A nationwide flood-frequency report (Sauer and others, 1983) which uses urban characteristics to adjust rural discharges to estimate urban discharges is available. Equations in that report were developed using a1l available U.S. Geological Survey urban data throughout the United States along with the respective rural discharge. The equations in that report should be applicable to urban Arkansas streams.

Three sets of equations are shown in that report for computing urban discharges. Two of them uses seven parameters for estimating discharge and the other uses three parameters. Recent studies in the southeastern part of the Nation indicate that the three-parameter equation is biased in certain areas, whereas the seven parameter equation is not biased. The seven parameter equations are probably a good estimate of the urban flood frequency in Arkansas and are shown below. The following equations and definitions are excerpts from Sauer and others (1983):

Average standard error of regression, in percent

\begin{tabular}{|c|c|}
\hline $\begin{array}{l}\mathrm{UQ}_{2} \\
\mathrm{UQ}_{5} \\
\mathrm{UQ}_{10} \\
\mathrm{UQ}_{25} \\
\mathrm{UQ}_{50}\end{array}$ & 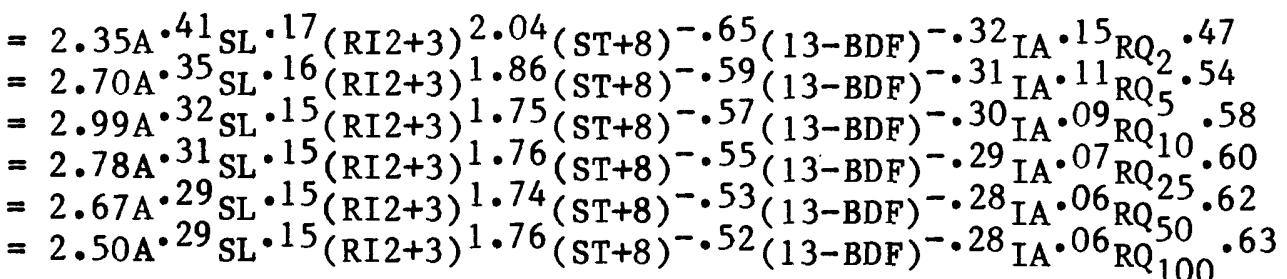 \\
\hline
\end{tabular}




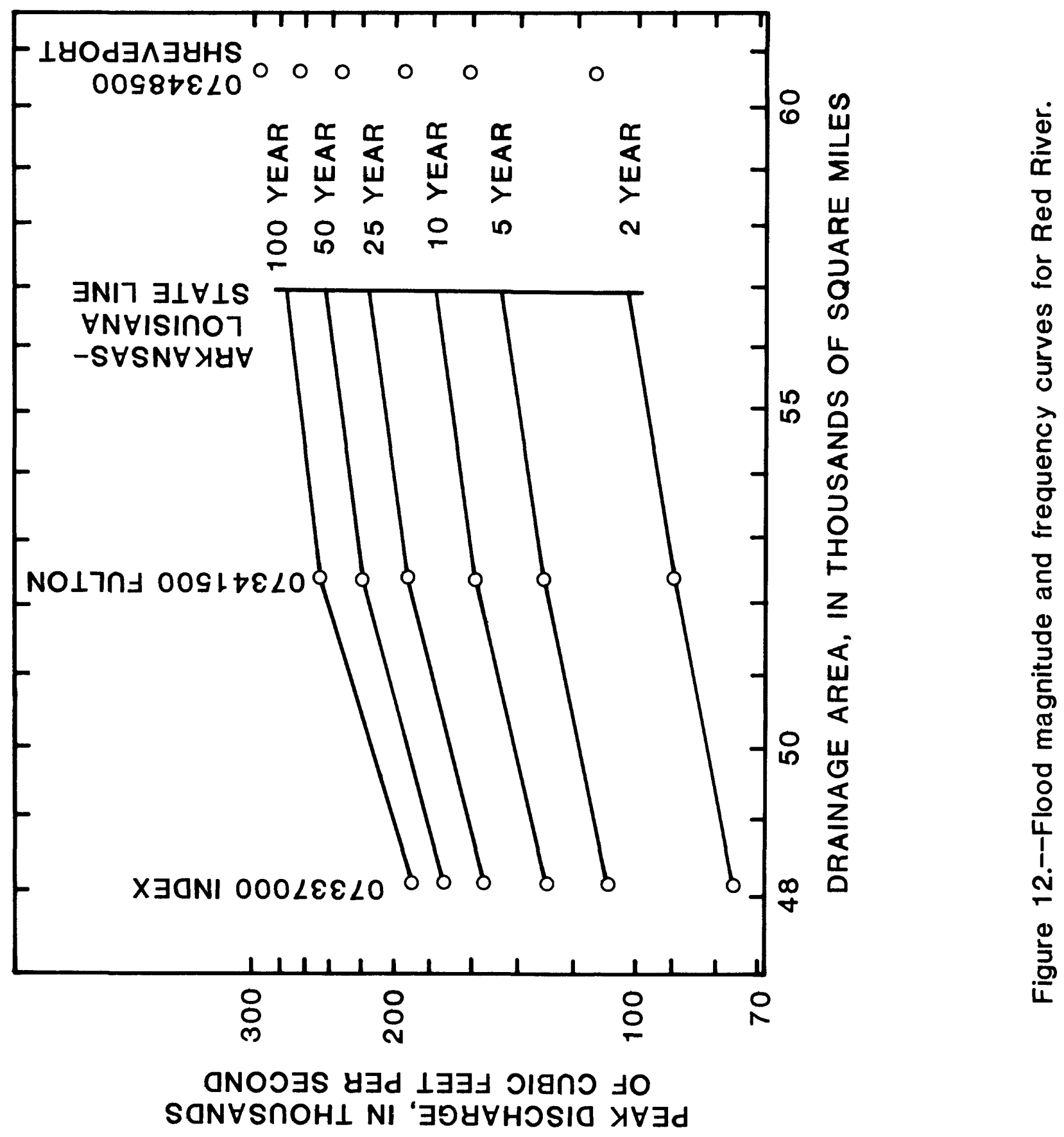




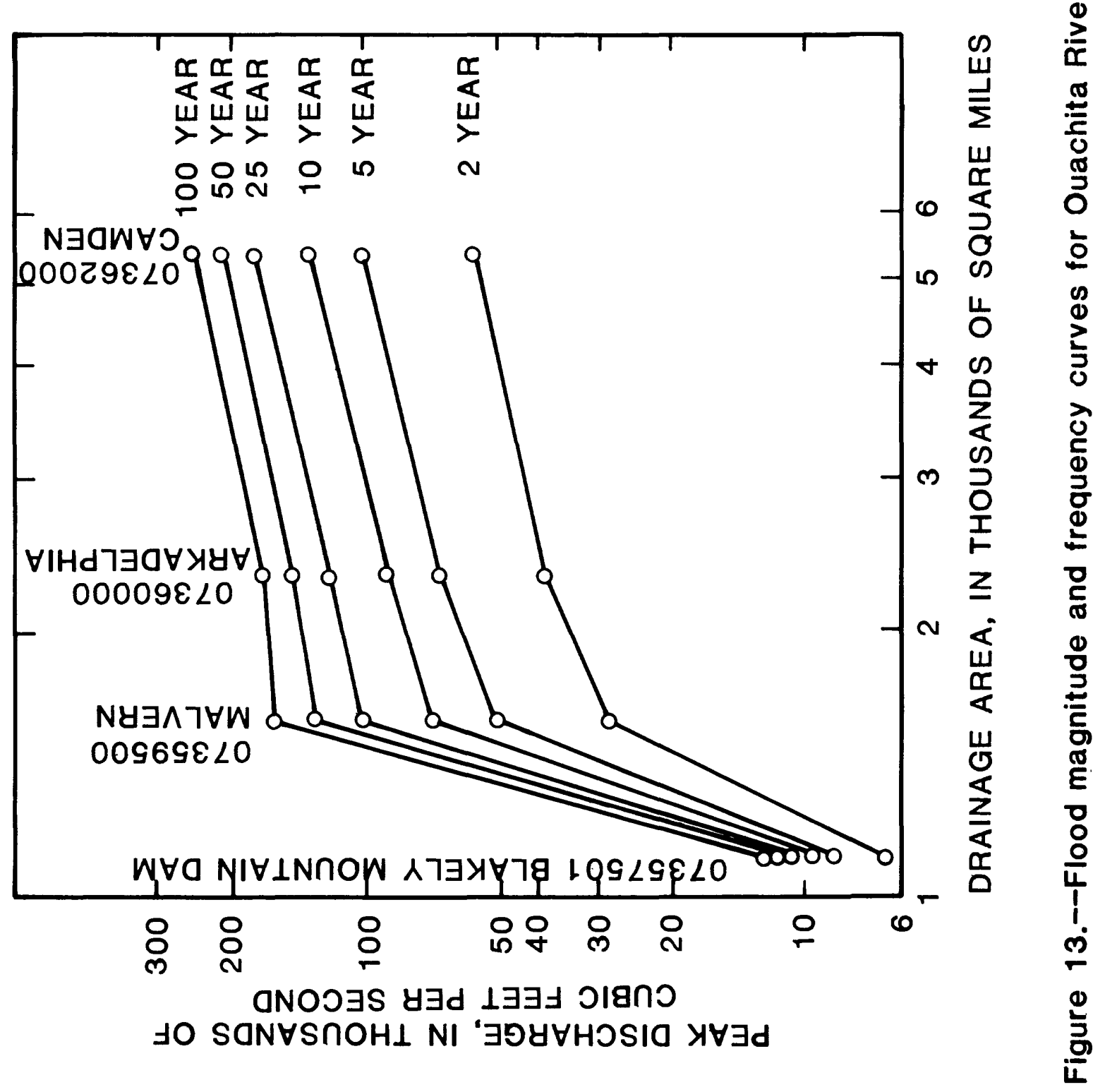


where,

$\mathrm{UQX}=$ The peak discharge, in $\mathrm{ft}^{3} / \mathrm{s}$, urban discharge for the urban watershed for recurrence interval, $x$.

$\mathrm{A}=$ The contributing drainage area, in $\mathrm{mi}^{2}$.

SL = The main channel slope, in feet per mile, measured between points which are 10 percent and 85 percent of the main channel length upstream from the study site. For sites where SL is greater than 70,70 is used in the equations.

RI2 = Rainfal1 intensity, in in., for the 2-hour 2-year occurrence. The rainfall intensity for the 2-hour, 2-year occurrence is shown on figure 14 (Weather Bureau, 1961).

ST = Basin storage, the percentage of the drainage basin occupied by lakes, reservoirs, swamps, and wetlands. In-channel storage of a temporary nature, resulting from detention ponds or roadway embankments, is not included in the computation of ST.

$\mathrm{BDF}=$ The basin development factor.

IA = The percentage of the drainage basin occupied by impervious surfaces, such as houses, buildings, streets, and parking lots.

$\mathrm{RQX}=$ The peak discharge, in $\mathrm{ft}^{3} / \mathrm{s}$, for an equivalent rural drainage basin in the same hydrologic area as the urban basin, and for recurrence interval $\mathrm{x}$.

The basin development factor (BDF) describes the conditions of the drainage system. The following description of the BDF and how it is computed is a quotation from Sauer and others (1983).

The most significant index of urbanization that results from this study is a basin development factor (BDF), which provides a measure of the efficiency of the drainage system. This parameter, which proved to be highly significant in the regression equations, can be easily determined from drainage maps and field inspections of the drainage basin. The basin is first divided into thirds. Then, within each third, four aspects of the drainage system are evaluated and each assigned a code as follows:

1. Channel improvements.--If channel improvements such as straightening, enlarging, deepening, and clearing are prevalent for the main drainage channels and principal tributaries (those that drain directly into the main channe 1), then a code of 1 is assigned. Any or all of these improvements would qualify for a code of 1 . To be considered prevalent, at least 50 percent of the main drainage channels and principal tributaries must be improved to some degree over natural conditions. If channel improvements are not prevalent, then a code of zero is assigned.

2. Channel linings.--If more than 50 percent of the length of the main drainage channels and principal tributaries has been lined with an impervious material, such as concrete, then a code of 1 is assigned to this aspect. 


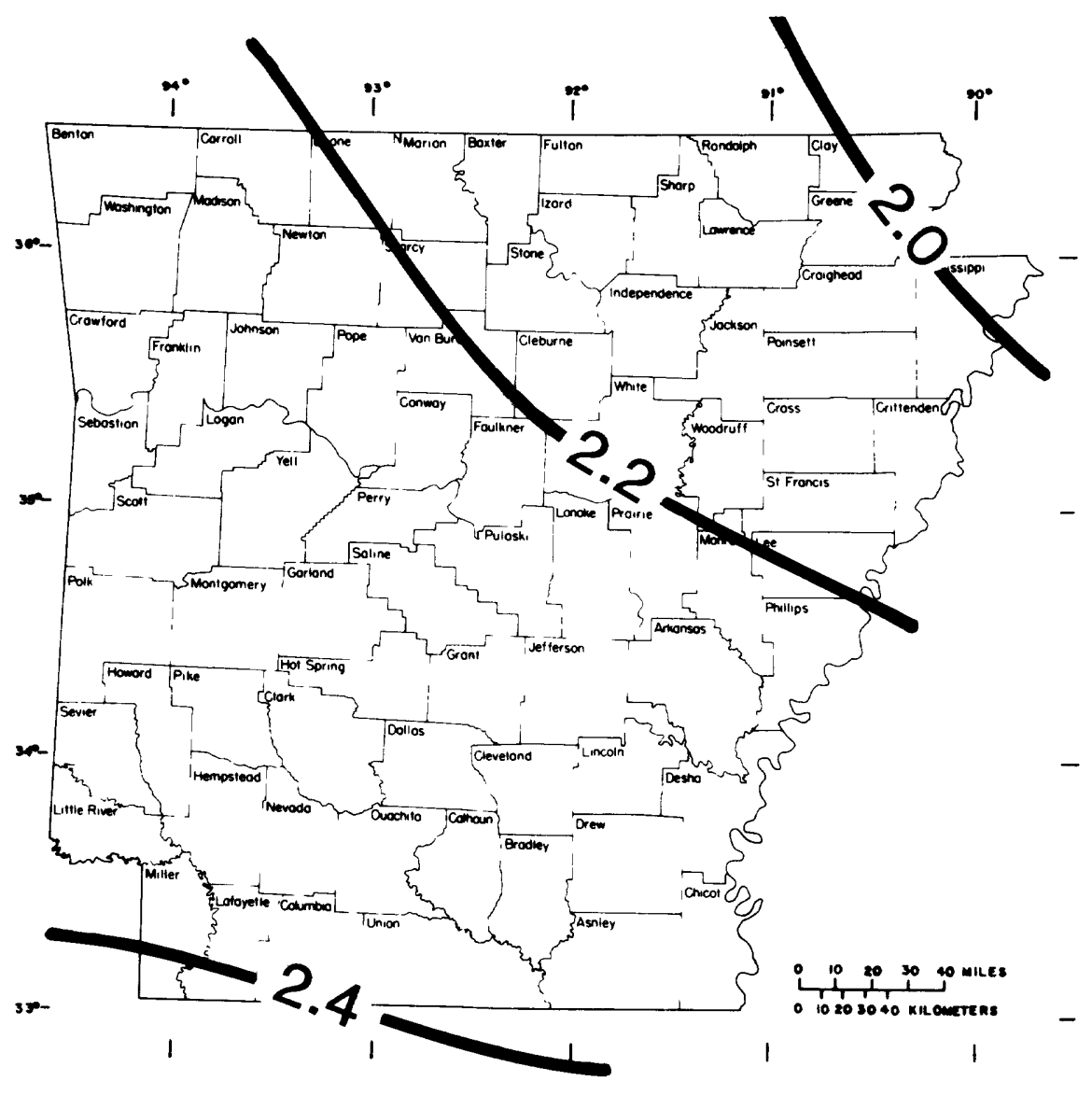

\section{EXPLANATION}

$-2.2-$ LINE OF EQUAL RAINFALL

INTENSITY (INCHES). INTERVAL 0.2 INCH

Figure 14.--Rainfall intensity for the 2-hour, 2-year occurrence (Weather Bureau Technical Paper No. 4). 
If less than 50 percent of these channels is lined, then a code of zero is assigned. The presence of channel linings would obviously indicate the presence of channel improvements as well. Therefore, this is an added factor and indicates a more highly developed drainage system.

3. Storm drains, or storm sewers.--Storm drains are defined as enclosed drainage structures (usually pipes), frequently used on the second tributaries where the drainage is received directly from streets or parking lots. Many of these drains empty into open channels; however, in some basins they empty into channels enclosed as box or pipe culverts. When more than 50 percent of the secondary tributaries within a subarea (third) consists of storm drains, then a code of 1 is assigned to this aspect; if less than 50 percent of the secondary tributaries consists of storm drains, then a code of zero is assigned. It should be noted that if 50 percent or more of the main drainage channels and principal tributaries are enclosed, then the aspects of channel improvements and channel linings would also be assigned a code of 1 .

4. Curb-and-gutter streets.--If more than 50 percent of a subarea (third) is urbanized (covered by residential, commercial, and/or industrial development), and if more than 50 percent of the streets and highways in the subarea are constructed with curbs and gutters then a code of 1 would be assigned to this aspect. Otherwise, it would receive a code of zero. Drainage from curb-andgutter streets frequently empties into storm drains.

The above guidelines for determining the various drainagesystem codes are not intended to be precise measurements. A certain amount of subjectivity will necessarily be involved. Field checking should be performed to obtain the best estimate. The BDF is the sum of the assigned codes; therefore, with three subareas (thirds) per basin, and four drainage aspects to which codes are assigned in each subarea, the maximum value for a fully developed drainage system would be 12 . Conversely, if the drainage system were totally undeveloped, then a BDF of zero would result. Such a condition does not necessarily mean that the basin is unaffected by urbanization. In fact, a basin could be partially urbanized, have some impervious area, have some improvement of secondary tributaries, and still have an assigned BDF of zero.

The BDF is a fairly easy index to estimate for an existing urban basin. The 50-percent guideline will usually not be difficult to evaluate because many urban areas tend to use the same design criteria, and therefore have similar drainage aspects, throughout. Also, the BDF is convenient for projecting future development. Obviously, full development and maximum urban effects on peaks would occur when BDF $=12$. Projections of full development or intermediate stages of development can usually be obtained from city engineers. 


\section{CONCLUSIONS}

Methods are presented for estimating the magnitude and frequency of peak discharges on streams in Arkansas. Flood data from 200 gaging stations were used to develop regression equations to estimate peak discharges with selected recurrence intervals from 2 to 100 years on streams that drain less than 3,000 $\mathrm{mi}^{2}$. The State was divided into two regions to improve the accuracy of the regression equations. Region A includes most of the Mississippi Alluvial Plain in Arkansas, with the exception of Crowleys Ridge. Region $B$ includes the rest of the State. Equations were developed separately for Regions $A$ and $B$.

The regression analysis for Region A indicated that size of drainage area, slope of the main channel, and length of the main channel were the most significant basin characteristics that affect the magnitude and frequency of floods. The standard error of these equations ranged from 28 to 40 percent. The regression analysis for Region $B$ indicated that size of drainage area, slope of the main channel, elevation of the basin, and mean annual precipitation were the most significant basin and climatic characteristics that affect the magnitude and frequency of floods. The standard error of these equations ranged from 33 to 42 percent.

An alternate procedure, which uses the hydraulic radius as one of the basin characteristics for computing flood frequency is described. Hydraulic radius has not been used in flood-frequency reports in the past because it is difficult to determine at ungaged sites. In order to determine the hydraulic radius at an ungaged site, a cross section of the stream valley and a stage-discharge relation must be estimated. A method to estimate a cross section from topographic maps and to compute a stage-discharge relation for that section is described.

Large regulated rivers such as the Red, Arkansas, White, Black, St. Francis, Mississippi, and Ouachita Rivers have floodflow characteristics that differ from those of smaller tributary streams and were treated individually. The discharge for the regulated period reflects the pattern of regulation. If the pattern of regulation changes, then the discharges will change.

A method is described for estimating the magnitude and frequency of peak discharges on streams for urban areas in Arkansas. This method is based on a nationwide U.S. Geological Survey flood-frequency report which uses urban characteristics to adjust rural discharge to estimate urban discharges. 
Farnsworth, R. K., Thompson, E. S., and Peck, E. L., 1982, Evaporation atlas for the contiguous 48 United States: National Oceanic and Atmospheric Administration Technical Report NWS 33, 26 p.

Fenneman, N. M., 1938, Physiography of eastern United States: New York, McGraw-Hi11, 724 p.

Freiwald, D. A., 1985, Average annual precipitation and runoff for Arkansas, 1951-80: U.S. Geological Survey Water-Resources Investigations Report 84-4363, 1 sheet.

Hardison, C. H., 1969, Accuracy of streamflow characteristics: U.S. Geological Survey Professional Paper 650-D, p. D210-214.

Hunrichs, R. A., 1983, Identification and classification of perennial streams of Arkansas: U.S. Geological Survey Water-Resources Investigations Report 83-4063, 1 sheet.

Hydrology subcommittee of the Interagency Advisory Committee on water data, 1982, Guidelines for determining flood frequency: U.S. Geological Survey Bulletin 17B, Office of Water Data Coordination, Reston, Virginia.

Jarrett, R. D., 1985, Determination of roughness coefficients for streams in Colorado: U.S. Geological Survey Water-Resources Investigations Report $85-4004,54$ p.

Landers, M. N., 1985, Floodflow frequency of streams in the alluvial plain of the lower Mississippi River in Mississippi, Arkansas, and Louisiana: U.S. Geological Survey Water-Resources Investigations Report 85-4150, 21 p.

Patterson, J. L., 1961, Floods in Arkansas, magnitude and frequency: U.S. Geological Survey open-file report, $128 \mathrm{p}$.

1971, Floods in Arkansas, magnitude and frequency characteristics through 1968, Arkansas Geological Commission Water Resources Circular No. 11,21 p.

Riggs, H. C., 1976, A simplified slope-area method for estimating flood discharges in natural streams: U.S. Geological Survey Journal of Research, v. 4, no. 3, p. 285-191.

Sauer, V. B., and others, 1983, Flood characteristics of urban watersheds in the United States: U.S. Geological Survey Water Supply Paper 2207, 63 p.

Weather Bureau, 1961, Rainfall frequency atlas of the United States: U.S. Department of Commerce Technical Paper no. 40, 61 p. 